\title{
First-Order Regular and Degenerate Identification Differential Problems
}

\author{
A. Favini, ${ }^{1}$ A. Lorenzi, ${ }^{2}$ and H. Tanabe $^{3}$ \\ ${ }^{1}$ Dipartimento di Matematica, Università di Bologna, Piazza di Porta S. Donato 5, 40126 Bologna, Italy \\ ${ }^{2}$ Dipartimento di Matematica "F. Enriques", Università degli Studi di Milano, Via Saldini 50, 20133 Milano, Italy \\ ${ }^{3}$ Hirai Sanso 12-13, Takarazuka 665-0817, Japan
}

Correspondence should be addressed to A. Favini; favini@dm.unibo.it

Received 19 August 2013; Accepted 11 February 2014

Academic Editor: Baoxiang Wang

Copyright (C) 2015 A. Favini et al. This is an open access article distributed under the Creative Commons Attribution License, which permits unrestricted use, distribution, and reproduction in any medium, provided the original work is properly cited.

We are concerned with both regular and degenerate first-order identification problems related to systems of differential equations of weakly parabolic type in Banach spaces. Several applications to partial differential equations and systems will be given in a subsequent paper to show the fullness of our abstract results.

\section{Introduction}

The basic aim of this paper consists in extending the results in [1] and in solving some identification problems in a product space $X \times X$, where $X$ is a Banach space $X$, endowed with a norm $\|\cdot\|$, related to systems of two possibly degenerate first-order differential equations in time. More precisely, we will consider the differential problem: determine a pair of functions $y=\left(y_{1}, y_{2}\right):[0, \tau] \rightarrow X \times X$ and $f:[0, \tau] \rightarrow \mathbb{R}$, $\tau \in \mathbb{R}_{+}$, such that

$$
\begin{gathered}
\left(M_{1,1} y_{1}\right)^{\prime}(t)+\left(M_{1,2} y_{2}\right)^{\prime}(t)+L_{1,1} y_{1}(t) \\
+L_{1,2} y_{2}(t)=f(t) z_{1}, \quad 0 \leq t \leq \tau, \\
\left(M_{2,1} y_{1}\right)^{\prime}(t)+\left(M_{2,2} y_{2}\right)^{\prime}(t)+L_{2,1} y_{1}(t) \\
+L_{2,2} y_{2}(t)=f(t) z_{2}, \quad 0 \leq t \leq \tau, \\
M_{1,1} y_{1}(0)+M_{1,2} y_{2}(0)=M_{1,1} y_{1,0}+M_{1,2} y_{2,0}, \\
M_{2,1} y_{1}(0)+M_{2,2} y_{2}(0)=M_{2,1} y_{1,0}+M_{2,2} y_{2,0}, \\
\Psi_{1}\left[M_{1,1} y_{1}(t)+M_{1,2} y_{2}(t)\right] \\
+\Psi_{2}\left[M_{2,1} y_{1}(t)+M_{2,2} y_{2}(t)\right]=g(t), \quad 0 \leq t \leq \tau .
\end{gathered}
$$

In particular, the regular choice corresponds to the case where

$$
M_{1,1}=M_{2,1}=I, \quad M_{1,2}=M_{2,2}=0 .
$$

The starting point for this paper is provided by [1] (cf. also [2]) where the identification degenerate problem

$$
\begin{gathered}
D_{t} M y(t)+L y(t)=f(t) z, \quad 0 \leq t \leq \tau, \\
(M y)(0)=M y_{0}, \\
\Phi[y(t)]=g(t), \quad 0 \leq t \leq \tau,
\end{gathered}
$$

is studied in the Banach space $X$ under assumptions of weak parabolicity (cf. [3]) on the linear closed linear operators $L$ and $M$.

Existence of solutions to evolution equations with matrix-valued operator coefficients has been considered very recently by Engel [4] where no degeneration is involved.

Abstract systems of parabolic equations of relevant importance in applications are described in the monograph by Yagi [5].

Here we study both the regular and the degenerate cases using the basic results in [1] and the different methods introduced therein. This will allow us to handle, in particular, systems of elliptic-parabolic equations and systems of degenerate parabolic equations in different functional spaces.

We indicate now the plan of the paper. 
In Section 2, we will consider an identification problem for first-order regular systems of differential equations.

In Section 3, we will deal with identification problems for first-order in time systems of PDE's, treating in particular some nonstandard boundary conditions.

Section 4 contains the main contributions to the degenerate case. The first two results-Theorems 14 and 18-can be easily extended to matrix-valued operators of the form

$$
\left[\begin{array}{ll}
A & B \\
C & D
\end{array}\right]
$$

where operators $A$ and $C$ map from $X$ to $X$ and $Y$, respectively, while operators $B$ and $D$ map from $Y$ to $X$ and $Y$, respectively, and $Y$ being a suitable Banach space.

Different levels of degeneration for operator matrices not entering the time derivative will be considered and corresponding conditions for the solvability of the related identification problems will be given.

Section 5 contains some extensions of the basic results in [1], which will play a key role for the present paper.

Section 6 contains a number of applications to systems of PDE's enlightening the concrete applications and the strict conditions to be satisfied by the single equations to guarantee both existence, uniqueness, and the regularity of solutions.

The Appendix section describes a general approach to inverse problems using a perturbation theory of generators. In such a way, the inverse problem under consideration is reduced to a direct problem with a new generator.

A number of specific examples illustrate the extension and the strength of this method.

\section{Identification Problems for First-Order Regular Differential Systems in Banach Spaces}

Let $X$ be a complex Banach space with norm $\|\cdot\|$ and let

$$
\Sigma_{\alpha}=\left\{\lambda \in \mathbb{C}: \operatorname{Re} \lambda \geq-c_{0}(1+|\operatorname{Im} \lambda|)^{\alpha}\right\}, \quad c_{0}>0 .
$$

The space $X \times X$ is endowed with the product norm.

Let $A: \mathscr{D}(A) \subset X \rightarrow X, B: \mathscr{D}(B) \subset X \rightarrow X$, $C: \mathscr{D}(C) \subset X \rightarrow X, D: \mathscr{D}(D) \subset X \rightarrow X$ be four closed linear operators satisfying the following properties, where $\rho(L)$ denotes the resolvent set of a linear closed operator $L$ : $\mathscr{L}(B) \subset X \times X:$

(H1) $\mathscr{D}(A) \subset \mathscr{D}(C)$ and $\mathscr{D}(D) \subset \mathscr{D}(B)$;

$(\mathrm{H} 2)\left\|(\lambda+A)^{-1}\right\|_{\mathscr{L}(X)} \leq C_{1}(1+|\lambda|)^{-\beta_{1}}$ for all $\lambda \in \Sigma_{\alpha}$;

(H3) $\left\|(\lambda+D)^{-1}\right\|_{\mathscr{L}(X)} \leq C_{2}(1+|\lambda|)^{-\beta_{2}}$ for all $\lambda \in \Sigma_{\alpha}$;

(H4) $\left\|C(\lambda+A)^{-1}\right\|_{\mathscr{L}(X)} \leq C_{3}(1+|\lambda|)^{-\sigma_{1}}$ for all $\lambda \in \Sigma_{\alpha}$;

(H5) $\left\|B(\lambda+D)^{-1}\right\|_{\mathscr{L}(X)} \leq C_{4}(1+|\lambda|)^{-\sigma_{2}}$ for all $\lambda \in \Sigma_{\alpha}$;

(H6) $\beta_{j} \in(0,1], \sigma_{j} \in \mathbb{R}, j=1,2, \sigma_{1}+\sigma_{2} \in \mathbb{R}_{+}$;

(H7) $\Phi_{j} \in X^{*}, j=1,2$.
We consider here the problem consisting in recovering the unknown scalar function $f:[0, T] \rightarrow \mathbb{R}$ in the following differential system in $X \times X$ :

$$
D_{t}\left[\begin{array}{l}
x(t) \\
y(t)
\end{array}\right]+\left[\begin{array}{ll}
A & B \\
C & D
\end{array}\right]\left[\begin{array}{l}
x(t) \\
y(t)
\end{array}\right]=f(t)\left[\begin{array}{l}
z_{1} \\
z_{2}
\end{array}\right], \quad 0 \leq t \leq \tau,
$$

subject to the initial conditions

$$
x(0)=x_{0}, \quad y(0)=y_{0},
$$

and to the additional information

$$
\Phi_{1}[x(t)]+\Phi_{2}[y(t)]=g(t), \quad 0 \leq t \leq \tau .
$$

First we consider the following resolvent system in $X \times X$, where $\lambda \in \Sigma_{\alpha}$ and $w, z \in X$ :

$$
\begin{aligned}
& (\lambda+A) x+B y=z, \\
& C x+(\lambda+D) y=w
\end{aligned}
$$

that we rewrite in the vector form:

$$
\left[\begin{array}{cc}
\lambda+A & B \\
C & \lambda+D
\end{array}\right]\left[\begin{array}{l}
x \\
y
\end{array}\right]=\left[\begin{array}{c}
z \\
w
\end{array}\right]
$$

Note that according to our assumption (H3) we get

$$
\begin{gathered}
y=-(\lambda+D)^{-1} C x+(\lambda+D)^{-1} w \\
{\left[I-B(\lambda+D)^{-1} C(\lambda+A)^{-1}\right](\lambda+A) x=z-B(\lambda+D)^{-1} w .}
\end{gathered}
$$

Observe now that

$$
\begin{aligned}
& \left\|B(\lambda+D)^{-1} C(\lambda+A)^{-1}\right\|_{\mathscr{L}(X)} \\
& \quad \leq\left\|B(\lambda+D)^{-1}\right\|_{\mathscr{L}(X)}\left\|C(\lambda+A)^{-1}\right\|_{\mathscr{L}(X)} \\
& \quad \leq C_{3} C_{4}(1+|\lambda|)^{-\sigma_{1}-\sigma_{2}}<\frac{1}{2},
\end{aligned}
$$

if

$$
|\lambda|>\left(2 C_{3} C_{4}\right)^{1 /\left(\sigma_{1}+\sigma_{2}\right)}-1
$$

Then for all $\lambda \in \Sigma_{\alpha} \cap{\overline{B\left(0, r_{0}\right)}}^{c}=: S\left(\alpha, r_{0}\right)$ the linear operator $I-B(\lambda+D)^{-1} C(\lambda+A)^{-1}$ admits an inverse $R(\lambda)$ in $\mathscr{L}(X)$ satisfying the estimate

$$
\left\|\left[I-B(\lambda+D)^{-1} C(\lambda+A)^{-1}\right]^{-1}\right\|_{\mathscr{L}(X)} \leq 2 .
$$

Hence the solution to system (9) is given by

$$
\begin{aligned}
x= & (\lambda+A)^{-1} R(\lambda) z-(\lambda+A)^{-1} R(\lambda) B(\lambda+D)^{-1} w, \\
y= & -(\lambda+D)^{-1} C(\lambda+A)^{-1} R(\lambda) z \\
& +(\lambda+D)^{-1}\left[I+C(\lambda+A)^{-1} R(\lambda) B(\lambda+D)^{-1}\right] w .
\end{aligned}
$$


Therefore, for all $\lambda \in S\left(\alpha, r_{0}\right)$, we deduce the estimates

$$
\begin{aligned}
\|x\| \leq & C_{5}(1+|\lambda|)^{-\beta_{1}}\|z\|+C_{6}(1+|\lambda|)^{-\beta_{1}-\sigma_{2}}\|w\|, \\
\|y\| \leq & C_{7}(1+|\lambda|)^{-\beta_{2}-\sigma_{1}}\|z\| \\
& +C_{8}(1+|\lambda|)^{-\beta_{2}}\left\{1+(1+|\lambda|)^{-\sigma_{1}-\sigma_{2}}\right\}\|w\| .
\end{aligned}
$$

Since $\sigma_{1}+\sigma_{2}>0$, we conclude that the operator matrix

$$
\mathscr{A}=\left[\begin{array}{ll}
A & B \\
C & D
\end{array}\right], \quad \mathscr{D}(\mathscr{A})=\mathscr{D}(A) \times \mathscr{D}(D),
$$

satisfies the following bound in the product space $X \times X$ :

$$
\left\|(\lambda+\mathscr{A})^{-1}\right\|_{\mathscr{L}(X \times X)} \leq C_{9}|\lambda|^{-\beta}, \quad \lambda \in S\left(\alpha, r_{0}\right),
$$

where

$$
\beta=\min \left\{\beta_{1}, \beta_{2}, \beta_{1}+\sigma_{2}, \beta_{2}+\sigma_{1}\right\}
$$

Remark 1. If operators $B$ and $C$ are bounded, then $\sigma_{j}=\beta_{j}$, $j=1,2$; thus, $\sigma_{1}+\sigma_{2}>0$ is trivially verified.

Remark 2. If $\mathscr{D}(B) \simeq \mathscr{D}(D)$, then $\sigma_{2}=\beta_{2}-1 \leq 0$. Indeed, for any $\lambda_{0} \in-\rho(D)$ it follows

$$
\begin{aligned}
& \left\|\left(\lambda_{0}+D\right)(\lambda+D)^{-1}\right\|_{\mathscr{L}(X)} \\
& \quad \leq\left\|\lambda_{0}(\lambda+D)^{-1}+I-\lambda(\lambda+D)^{-1}\right\|_{\mathscr{L}(X)} \\
& \quad \leq C_{2}\left|\lambda_{0}\right|(1+|\lambda|)^{-\beta_{2}}+1+C_{2}|\lambda|(1+|\lambda|)^{-\beta_{2}} \\
& \quad \leq C_{10}(1+|\lambda|)^{1-\beta_{2}} .
\end{aligned}
$$

Moreover, the closed graph theorem implies $B\left(\lambda_{0}+D\right)^{-1} \epsilon$ $\mathscr{L}(X)$. This and the previous estimate imply

$$
\left\|B(\lambda+D)^{-1}\right\|_{\mathscr{L}(X)} \leq C_{11}(1+|\lambda|)^{1-\beta_{2}}
$$

The relation $\sigma_{2} \leq 0$, along with $\mathrm{H} 9$, implies $\sigma_{1}>-\sigma_{2}=1-$ $\beta_{2} \geq 0$. Consequently, the set relations $\mathscr{D}(C) \simeq \mathscr{D}(A)$ and $\mathscr{D}(B) \simeq \mathscr{D}(D)$ cannot occur simultaneously.

Taking the results in [1] into account (reported and improved a bit in Section 5), we deduce the following Theorem 3.

Theorem 3. Let $-A$ and $-D$ be the generators of two differentiable (not necessarily densely defined) semigroups of parabolic type in the complex Banach space X satisfying (H1)-(H3). Let $B$ and $C$ be two linear closed operators satisfying properties (H4)-(H6) and let $\Phi_{j}, j=1,2$, be two linear functionals with properties (H7). Let $(\alpha, \beta, \theta)$ be a triplet of real numbers such that (cf. (19))

$$
\alpha+\beta>\frac{3}{2}, \quad 2-\alpha-\beta<\theta<\alpha+\beta-1
$$

Let the data $\left(g, z_{1}, z_{2}\right)$ satisfy the properties

$$
\begin{gathered}
g \in C^{1+\theta}([0, \tau] ; * * * \mathbb{C} * * *), \quad z_{1} \in \mathscr{D}(A), \\
z_{2} \in \mathscr{D}(D), \quad * * * \Phi_{1}\left[z_{1}\right]+\Phi_{2}\left[z_{2}\right] \neq 0, * * * \\
\left(A u_{0}+B v_{0}, C u_{0}+D v_{0}\right) \in \mathscr{D}(A) \times \mathscr{D}(D), \\
g(0)=* * * \Phi_{1}\left[u_{0}\right]+\Phi_{2}\left[v_{0}\right] . * * *
\end{gathered}
$$

Then the identification problem

$$
\begin{gathered}
u^{\prime}(t)=-A u(t)-B v(t)+f(t) z_{1}, \quad 0 \leq t \leq \tau, \\
v^{\prime}(t)=-C u(t)-D v(t)+f(t) z_{2}, \quad 0 \leq t \leq \tau, \\
u(0)=u_{0}, \quad v(0)=v_{0} \\
\Phi_{1}[u(t)]+\Phi_{2}[v(t)]=g(t), \quad 0 \leq t \leq \tau
\end{gathered}
$$

admits a unique global solution $(u, v, f) \in\left[C^{1+\theta}([0, \tau] ; X) \cap\right.$ $\left.C^{\theta}([0, \tau], \mathscr{D}(A))\right] \times\left[C^{1+\theta}([0, \tau] ; X) \cap C^{\theta}([0, \tau], \mathscr{D}(D))\right] \times$ $C^{\theta}([0, \tau] ; \mathbb{C})$.

Remark 4. If $C=k A$ for some $k \in \mathbb{R}_{+}$and $B \in \mathscr{L}(X)$, then $\sigma_{1}=\beta_{1}-1, \sigma_{2}=\beta_{2}$. Thus $\sigma_{1}+\sigma_{2}=\beta_{1}+\beta_{2}-1>0$ implies $\beta_{1}+\beta_{2}>1$. In this case, owing to (19), we have $\beta=\beta_{1}+\beta_{2}-1$, so that we need to require $\alpha+\beta_{1}+\beta_{2}>5 / 2$.

In most applications, $\alpha=1$, so that this relation reduces to $\beta_{1}+\beta_{2}>3 / 2$.

\section{Identification Problems for Regular First-Order in Time Systems of PDE's}

In this section, we will deal with some identification problems related to systems of PDE's.

Problem 5. Let $\Omega$ be a bounded domain in $\mathbb{R}^{n}$ with a $C^{\infty}$ boundary $\partial \Omega$.

We want to recover the scalar function $f:[0, \tau] \rightarrow \mathbb{R}$ in the initial-boundary value problem:

$$
\begin{aligned}
& D_{t} u(t, x)+A\left(x, D_{x}\right) u(t, x) \\
& +B\left(x, D_{x}\right) v(t, x)=f(t) z_{1}(x), \quad(t, x) \in(0, \tau) \times \Omega, \\
& D_{t} v(t, x)+C\left(x, D_{x}\right) u(t, x) \\
& +D\left(x, D_{x}\right) v(t, x)=f(t) z_{2}(x), \quad(t, x) \in(0, \tau) \times \Omega, \\
& u(x, 0)=u_{0}(x), \quad v(x, 0)=v_{0}(x), \quad x \in \Omega, \\
& a(x) D_{v} u(t, x)+\alpha(x) \cdot \nabla u(t, x)+b(x) u(t, x)=0, \\
& \quad(t, x) \in(0, \tau) \times \partial \Omega, \\
& c(x) D_{v} v(t, x)+\beta(x) \cdot \nabla v(t, x)+d(x) u(t, x)=0, \\
& \quad(t, x) \in(0, \tau) \times \partial \Omega,
\end{aligned}
$$


under the additional information

$$
\int_{\Omega}\left[\eta_{1}(x) u(x, t)+\eta_{2}(x) v(x, t)\right] d x=g(t), \quad t \in[0, \tau] .
$$

Here

$$
\begin{aligned}
& -A\left(x, D_{x}\right)=\sum_{i, j=1}^{n} a_{i, j}(x) D_{x_{i}} D_{x_{j}}+\sum_{i=1}^{n} a_{i}(x) D_{x_{i}}+a_{0}(x), \\
& -D\left(x, D_{x}\right)=\sum_{i, j=1}^{n} \tilde{a}_{i, j}(x) D_{x_{i}} D_{x_{j}}+\sum_{i=1}^{n} \widetilde{a}_{i}(x) D_{x_{i}}+\tilde{a}_{0}(x)
\end{aligned}
$$

are two second-order linear elliptic differential operators with real-valued $C^{\infty}$-coefficients on $\bar{\Omega}$ such that

$$
\begin{array}{r}
a_{i, j}(x)=a_{j, i}(x), \quad \sum_{i, j=1}^{n} a_{i, j}(x) \xi_{i} \xi_{j} \geq c_{0}|\xi|^{2}, \\
(x, \xi) \in \bar{\Omega} \times \mathbb{R}^{n}, \\
\tilde{a}_{i, j}(x)=\tilde{a}_{j, i}(x), \quad \sum_{i, j=1}^{n} \tilde{a}_{i, j}(x) \xi_{i} \xi_{j} \geq c_{0}|\xi|^{2}, \\
(x, \xi) \in \bar{\Omega} \times \mathbb{R}^{n},
\end{array}
$$

$c_{0}$ being a positive constant.

Assume for the time being that $B\left(x, D_{x}\right)$ and $C\left(x, D_{x}\right)$ are multiplication operators defined, respectively, by two $C^{\infty}(\bar{\Omega})$ functions $r$ and $s$. In this case we have $\sigma_{j}=\beta_{j}$.

Concerning the linear boundary differential operators defined by

$$
\widehat{A} u=a D_{y} u+\alpha \cdot \nabla u+b u, \quad \widehat{D} u=\widetilde{a} D_{y} u+\widetilde{\alpha} \cdot \nabla u+\widetilde{b} u,
$$

assume that $a, \tilde{a}, b, \widetilde{b}$ and $\alpha, \widetilde{\alpha}$ are real-valued $C^{\infty}$ functions and vector fields on $\partial \Omega$ such that $\mathscr{T} u=\alpha \cdot \nabla u$. and $\widetilde{\mathscr{T}} v=\widetilde{\alpha} \cdot \nabla v$ are real $C^{\infty}$-tangential operators on $\partial \Omega, D_{\nu}$ and $D_{\widetilde{\nu}}$ standing for the conormal derivatives associated with the matrices $\left(a_{i, j}(x)\right)$ and $\left(\widetilde{\alpha}_{i, j}(x)\right)$, respectively; that is,

$$
\begin{aligned}
& D_{\nu}=\left(\sum_{i, j=1}^{n} a_{i, j}(x) n_{i}(x) n_{j}(x)\right)^{-1} \sum_{i, j=1}^{n} a_{i, j}(x) n_{i}(x) D_{x_{i}}, \\
& D_{\widetilde{v}}=\left(\sum_{i, j=1}^{n} \tilde{a}_{i, j}(x) n_{i}(x) n_{j}(x)\right)^{-1} \sum_{i, j=1}^{n} \tilde{a}_{i, j}(x) n_{i}(x) D_{x_{i}},
\end{aligned}
$$

$n(x)$ denoting the unit outward normal vector to $\partial \Omega$ at $x$.

Assume further (cf. page 515 in [6]) that the vector field $\alpha$ (resp., $\widetilde{\alpha}$ ) does not vanish on $\Gamma_{0}=\{x \in \partial \Omega: a(x)=0\}$ (resp., $\left.\widetilde{\Gamma}_{0}=\{x \in \partial \Omega: \widetilde{a}(x)=0\}\right)$ and the function $t \rightarrow a\left(x\left(t, x_{0}\right)\right)$ (resp., $t \rightarrow \tilde{a}\left(x\left(t, x_{0}\right)\right)$ ) has zeros of even order not greater than $2 \kappa_{1}$ (resp., $2 \kappa_{2}$ ) along the integral curve $x\left(t, x_{0}\right)$ of $\alpha$ (resp., $\widetilde{\alpha}$ ) passing through $x_{0} \in \Gamma_{0}$ (resp., $x_{0} \in \widetilde{\Gamma}_{0}$ ) at $t=0$. In other words, the so-called $(H)_{\delta}$-condition holds with $\delta=$ $\delta_{1}=1 /\left(1+2 \kappa_{1}\right)$ and $\delta=\delta_{2}=1 /\left(1+2 \kappa_{2}\right)$, respectively.

It is shown on page 516 in [6] that the operator $L_{1}$ and $L_{2}$ defined by

$$
\begin{aligned}
\mathscr{D}\left(L_{1}\right) & =\left\{u \in L^{2}(\Omega): A\left(x, D_{x}\right) u \in L^{2}(\Omega), \widehat{A} u=0\right\}, \\
L_{1} u & =-A\left(x, D_{x}\right) u, \quad u \in \mathscr{D}\left(L_{1}\right), \\
\mathscr{D}\left(L_{2}\right) & =\left\{v \in L^{2}(\Omega): D\left(x, D_{x}\right) v \in L^{2}(\Omega), \widehat{D} v=0\right\}, \\
L_{2} v & =-D\left(x, D_{x}\right) u, \quad v \in \mathscr{D}\left(L_{2}\right),
\end{aligned}
$$

satisfy in $L^{2}(\Omega)$ the resolvent estimates

$$
\begin{gathered}
\left\|\left(\lambda+L_{1}\right)^{-1}\right\|_{\mathscr{L}\left(L^{2}(\Omega)\right)} \leq C|\lambda|^{-\left(1+\delta_{1}\right) / 2}, \\
\left\|\left(\lambda+L_{2}\right)^{-1}\right\|_{\mathscr{L}\left(L^{2}(\Omega)\right)} \leq C|\lambda|^{-\left(1+\delta_{2}\right) / 2}
\end{gathered}
$$

for all complex $\lambda$ in a sector $|\arg \lambda| \leq \varphi$, with $\varphi \in(\pi / 2, \pi)$ and $|\lambda|$ suitable large.

We are in the subelliptic case:

$$
\beta=\min \left\{\beta_{1}, \beta_{2}\right\}=\min \left\{\frac{1+\delta_{1}}{2}, \frac{1+\delta_{2}}{2}\right\}>\frac{1}{2} .
$$

Choose now $\theta \in(1-\beta, \beta)$ and make the following assumptions:

$$
\begin{gathered}
z_{j} \in \mathscr{D}\left(L_{j}\right), \quad \eta_{j} \in L^{2}(\Omega), \quad j=1,2, \\
u_{0} \in \mathscr{D}\left(L_{1}\right), \quad v_{0} \in \mathscr{D}\left(L_{2}\right), \\
A(\cdot, D) u_{0}+r(\cdot) v_{0} \in \mathscr{D}\left(L_{1}\right), \\
s(\cdot) u_{0}+D(\cdot, D) v_{0} \in \mathscr{D}\left(L_{2}\right), \\
g \in C^{1+\theta}([0, \tau] ; \mathbb{R}), \\
g(0)=\int_{\Omega}\left[\eta_{1}(x) u_{0}(x)+\eta_{2}(x) v_{0}(x)\right] d x \neq 0, \\
\int_{\Omega}\left[\eta_{1}(x) z_{1}(x)+\eta_{2}(x) z_{2}(x)\right] d x \neq 0 .
\end{gathered}
$$

Then our identification problem admits a unique global solution $(u, v, f) \in\left[C^{1+\theta}\left([0, \tau] ; L^{2}(\Omega)\right) \cap C^{\theta}\left([0, \tau], \mathscr{D}\left(L_{1}\right)\right)\right] \times$ $\left[C^{1+\theta}\left([0, \tau] ; L^{2}(\Omega)\right) \cap C^{\theta}\left([0, \tau], \mathscr{D}\left(L_{2}\right)\right)\right] \times C^{\theta}([0, \tau] ; \mathbb{R})$.

We consider now the more general case where $C\left(x, D_{x}\right)=$ $k A\left(x, D_{x}\right), k \in \mathbb{R}_{+}$, and $B$ is the multiplication operator by $s \in C^{\infty}(\bar{\Omega})$.

From Remark 4 we get $\beta=\beta_{1}+\beta_{2}-1$, so that $\alpha+\beta>3 / 2$ reduces to $\beta_{1}+\beta_{2}>3 / 2$, that is, to $\delta_{1}+\delta_{2}>1$. Since

$$
\delta_{1}+\delta_{2}=\frac{1}{1+2 \kappa_{1}}+\frac{1}{1+2 \kappa_{2}}<1, \quad \text { if } \kappa_{1}, \kappa_{2} \in \mathbb{N} \backslash\{0\},
$$


we are compelled to require that either of $\delta_{1}$ or $\delta_{2}$ must coincide with 1 .

Note that, if $\delta_{2}=1$, then the corresponding boundary value problem is elliptic and this holds if and only if $\widetilde{a}(x) \neq 0$ for all $x \in \partial \Omega$ (cf. [7], on page 515). In this case $\beta=\beta_{1}=$ $\left(1+\delta_{1}\right) / 2>1 / 2$ so that $1-\beta=\left(1-\delta_{1}\right) / 2$.

Choose now $\theta \in\left(\left(1-\delta_{1}\right) / 2,\left(1+\delta_{1}\right) / 2\right)$ and make the same assumptions as in the previous case, except for $\left(u_{0}, v_{0}\right)$. The related condition has to be changed to the following:

$$
\begin{gathered}
A(\cdot, D) u_{0}+s(\cdot) v_{0} \in \mathscr{D}\left(L_{1}\right), \\
k A(\cdot, D)(\cdot) u_{0}+D(\cdot, D) v_{0} \in \mathscr{D}\left(L_{2}\right) .
\end{gathered}
$$

Then the given identification problem admits a unique global solution $(u, v, f) \in\left[C^{1+\theta}\left([0, \tau] ; L^{2}(\Omega)\right) \cap C^{\theta}([0, \tau]\right.$, $\left.\left.\mathscr{D}\left(L_{1}\right)\right)\right] \times\left[C^{1+\theta}\left([0, \tau] ; L^{2}(\Omega)\right) \cap C^{\theta}\left([0, \tau], \mathscr{D}\left(L_{2}\right)\right)\right] \times C^{\theta}([0$, $\tau] ; \mathbb{R})$.

A corresponding result holds when $\delta_{2}=1$.

Problem 6. We note that in Problem 5 the domain of the operator-matrix is a product of domains. However, Corollary 31 allows to handle also decoupled domains. For this purpose, we will consider a problem related to a reaction diffusion model describing a man-environment epidemic system investigated in [8]. Such a model consists in a parabolic equation coupled with an ordinary differential equation via a boundary feedback operator (cf. also [7]).

In order to obtain stability results the authors linearize the model and arrive at the following evolution system, where $u(t, x)$ and $v(t, x)$ stand, respectively, for the concentration of the infection agent and the density of the infective population at time $t$ and point $x$ :

$$
\begin{array}{r}
D_{t} u(t, x)=\Delta u(t, x)-a(x) u(t, x)+f(t) z_{1}, \\
(t, x) \in(0,+\infty) \times \Omega, \\
D_{t} v(t, x)=c(x) u(t, x)-d(x) v(t, x)+f(t) z_{2}, \\
(t, x) \in(0, \tau) \times \Omega, \\
u(0, x)=u_{0}(x), \quad v(0, x)=v_{0}(x), \quad x \in \Omega, \\
D_{v} u(t, x)+\beta(x) u(t, x)=\int_{\Omega} k(x, y) v(t, y) d y, \\
(t, x) \in(0,+\infty) \times \partial \Omega,
\end{array}
$$

where $\Omega$ is a bounded domain in $\mathbb{R}^{n}$ with a smooth boundary $\partial \Omega, \Delta$ is the Laplacian, $a, c, d \in C(\bar{\Omega}), \beta \in C(\partial \Omega), k \in$ $L^{\infty}(\partial \Omega \times \Omega)$ are nonnegative functions, and $D_{v}$ denoted the outward normal derivative on $\partial \Omega$.

We define $E=C(\bar{\Omega}), X=E \times E$ and denote by $M_{h}$ the multiplication operator induced by the function $h$. Moreover, we introduce the operator matrix

$$
\begin{gathered}
A=\left[\begin{array}{cc}
\Delta-M_{a} & O \\
M_{c} & -M_{d}
\end{array}\right], \\
\mathscr{D}(A)=(u, v) \in X: u \in H^{2}(\Omega), \quad \Delta u \in E, \\
D_{\nu} u(x)+\beta(x) u(x)=\int_{\Omega} k(x, y) v(y) d y \quad \text { on } \partial \Omega .
\end{gathered}
$$

It can be proved (cf. [7, page 126]) that $A$ generates an analytic semigroup on $X$.

Consider then the identification problem consisting in finding a triplet $(u, v, f), f$ being a scalar function satisfying the direct Problem (38) as well as the additional condition:

$$
\sigma u(t, \bar{x})+\mu v(t, \tilde{x})=g(t), \quad t \in(0, \tau),
$$

where $z_{1}, z_{2}, u_{0}, v_{0} \in C(\bar{\Omega}), g \in C([0, \tau] ; \mathbb{R}), \bar{x}, \tilde{x}$ are fixed points in $\Omega,(\sigma, \mu) \in \mathbb{R}^{2} \backslash\{0\}$. As a consequence of [2, Theorem 3.2] we get

Proposition 7. Let $\theta \in(0,1), g \in C^{1+\theta}([0, \tau] ; \mathbb{R}),\left(u_{0}, v_{0}\right) \in$ $D_{-A}(1+\theta, \infty),\left(z_{1}, z_{2}\right) \in D_{-A}\left(\theta_{0}, \infty\right), \sigma u_{0}(\bar{x})+\mu v_{0}(\tilde{x})=$ $g(0), \sigma z_{1}(\bar{x})+\mu z_{2}(\tilde{x}) \neq 0$. Then the identification Problem (38), (41) admits a unique global strict solution $((u, v), f) \in$ $\left[C^{1+\theta}([0, \tau] ; X) \cap C^{\theta}([0, \tau] ; \mathscr{D}(A))\right] \times C^{\theta}([0, \tau] ; \mathbb{R})$.

Notice that the interpolation spaces $\mathscr{D}_{-A}(\theta, \infty)$ are well characterized both in an abstract form and for many boundary conditions (cf. $[4,9]$ ), but in this concrete case it seems to be difficult to translate them as on page 321 in [4]. Therefore, one can use the more restrictive assumptions $\left(u_{0}, v_{0}\right),\left(z_{1}, z_{2}\right) \in D(A)$ that can be easily checked.

Problem 8. We solve here an identification problem in Hölder spaces.

Let $\Omega$ be a bounded domain in $\mathbb{R}^{n}$ with a smooth boundary $\partial \Omega$. Then $C^{\nu+\omega}(\bar{\Omega}), \nu \in \mathrm{N} \backslash\{0\}$, and $\omega \in(0,1)$ denote the Banach space of all functions in $C^{\nu}(\bar{\Omega})$ whose derivatives of order $v$ are all Hölder continuous with exponent $\omega$. Such a space will be endowed with the natural norm $\|\cdot\|_{\nu+\omega}$

We introduce now some notation and assumption. As usual, $\widetilde{\alpha}=\left(\alpha_{1}, \ldots, \alpha_{n}\right) \in \mathbb{N}^{n}$ denotes a multi-index and we associate with it the monomial differential operator $D^{\widetilde{\alpha}}=$ $\prod_{j=1}^{n} D_{j}^{\alpha_{j}}, D_{j}=i^{-1} \partial / \partial x_{j}$. Finally, for any (fixed) $m \in \mathrm{N}$ and $\omega \in(0,1 / 3)$ let the functions $\widetilde{\alpha}: \bar{\Omega} \rightarrow \mathbb{C},|\widetilde{\alpha}| \leq 2 m$, satisfy, for some positive constant $M>1$, the relations:

$$
\begin{array}{r}
a_{\widetilde{\alpha}} \in C^{1+\omega}(\bar{\Omega} ; \mathbb{C}), \quad|\widetilde{\alpha}| \leq 2 m, \\
a_{\widetilde{\alpha}}(x) \in \mathbb{R}, \quad|\widetilde{\alpha}| \in\{0,2 m\}, \quad \forall x \in \bar{\Omega}, \\
M^{-1}|\xi|^{2 m} \leq \sum_{|\widetilde{\alpha}|=2 m} a_{\widetilde{\alpha}}(x) \xi^{\widetilde{\alpha}} \leq M|\xi|^{2 m}, \\
\forall x \in \bar{\Omega}, \quad \xi \in \mathbb{R}^{n} .
\end{array}
$$

Introduce now the linear operator $A$ in $X=C^{1+\omega}(\bar{\Omega})$ defined by

$$
\begin{gathered}
\mathscr{D}(A)=\left\{u \in C^{2 m+1+\omega}(\bar{\Omega}): D^{\widetilde{\alpha}} u=0\right. \\
\text { on } \partial \Omega,|\widetilde{\alpha}| \leq m-1\}, \\
A u(x)=\sum_{|\widetilde{\alpha}| \leq 2 m} a_{\widetilde{\alpha}}(x) D^{\tilde{\alpha}} u(x), \quad x \in \bar{\Omega}, u \in \mathscr{D}(A) .
\end{gathered}
$$


A remarkable result by von Wahl (cf. [10] on page 241) establishes the resolvent bound

$$
\begin{gathered}
\left\|(z+A)^{-1} u\right\|_{1+\omega} \leq C|z|^{-1+(1+\omega) /(2 m)}\|u\|_{1+\omega}, \\
\forall u \in C^{1+\omega}(\bar{\Omega}), \quad z \in \Sigma_{1} \backslash B(0, R),
\end{gathered}
$$

for a large $R>0$, where $\Sigma_{1}=\left\{z \in \mathbb{C}: \operatorname{Re} z \geq-c_{0}(1+\right.$ $|\operatorname{Im} z|)\}, c_{0}>0$.

For our application is not restrictive to assume that such a condition is satisfied for all $z \in \Sigma_{1}$. In this case the resolvent estimate

$$
\left\|(\lambda+A)^{-1}\right\|_{\mathscr{L}\left(C^{1+\omega}(\bar{\Omega})\right)} \leq C(1+|\lambda|)^{-\beta_{1}}, \quad \lambda \in \Sigma_{1}
$$

holds with $\beta_{1}=1-(1+\omega) /(2 m)$.

Let now $D$ be another linear differential operator of order $2 r$, with properties similar to those of $A$, defined by

$$
\begin{aligned}
& \mathscr{D}(D)=\left\{v \in C^{2 r+1+\omega}(\bar{\Omega}): D^{\widetilde{\alpha}} v=0 \text { on } \partial \Omega,|\widetilde{\alpha}| \leq r-1\right\}, \\
& D v(x)=\sum_{|\widetilde{\alpha}| \leq 2 r} d_{\widetilde{\alpha}}(x) D^{\widetilde{\alpha}} v(x), \quad x \in \bar{\Omega}, v \in \mathscr{D}(D),
\end{aligned}
$$

where $\omega \in(0,1 / 3)$.

Likewise as above, the spectral estimate

$$
\left\|(\lambda+D)^{-1}\right\|_{\mathscr{L}\left(C^{1+\omega}(\bar{\Omega})\right)} \leq C(1+|\lambda|)^{-\beta_{2}}, \quad \lambda \in \Sigma_{1}
$$

holds with $\beta_{2}=1-(1+\omega) /(2 r)$.

Notice that $\beta_{1}>1 / 2$ and $\beta_{2}>1 / 2$ imply $m \geq 2$ and $r \geq 2$.

Let now $B$ and $C$ be linear differential operators, with smooth coefficients (e.g., in $C^{1+\omega}(\bar{\Omega})$ ), defined by

$$
\begin{aligned}
& \mathscr{D}(B)=C^{p+1+\omega}(\bar{\Omega}), \mathscr{D}(C)=C^{q+1+\omega}(\bar{\Omega}), \\
& B=\sum_{|\widetilde{\beta}| \leq p} b_{\widetilde{\beta}}(x) D^{\tilde{\beta}}, \quad C=\sum_{|\widetilde{\gamma}| \leq q} c_{\tilde{\gamma}}(x) D^{\tilde{\gamma}},
\end{aligned}
$$

where $0 \leq p<2 r$ and $0 \leq q<2 m$.

In view of Satz II on page 239 in [10] we have

$\left\|B(z+D)^{-1}\right\|_{\mathscr{L}\left(C^{1+w}(\bar{\Omega})\right)} \leq C(1+|z|)^{-\sigma_{2}}, \quad z \in \Sigma_{1},|z|$ large $\left\|C(z+A)^{-1}\right\|_{\mathscr{L}\left(C^{1+\omega}(\bar{\Omega})\right)} \leq C(1+|z|)^{-\sigma_{1}}, \quad z \in \Sigma_{1},|z|$ large,

where

$$
\sigma_{1}=1-\frac{q+1+\omega}{2 m}, \quad \sigma_{2}=1-\frac{p+1+\omega}{2 r} .
$$

We introduce now the operator matrix

$$
\mathscr{D}(\mathscr{A})=\mathscr{D}(A) \times \mathscr{D}(D), \quad \mathscr{A}=\left[\begin{array}{ll}
A & B \\
C & D
\end{array}\right] .
$$

We can now apply Theorem 3 with $X=C^{1+\omega}(\bar{\Omega}), \alpha=1$ and

$$
\begin{gathered}
\omega \in\left(0, \frac{1}{3}\right), \quad r q+m p+(1+\omega)(r+m)<4 m r \\
\beta=\min \left\{1-\frac{1+\omega}{2 m}, 1-\frac{1+\omega}{2 r}, 2-\frac{1+\omega}{2 m}-\frac{p+1+\omega}{2 r}\right. \\
\left.2-\frac{1+\omega}{2 r}-\frac{q+1+\omega}{2 m}\right\}>\frac{1}{2}
\end{gathered}
$$

Under such hypotheses the identification problem

$$
\begin{gathered}
D_{t} u(t, x)+A u(t, x)+B v(t, x)=f(t) z_{1}(x), \\
(t, x) \in(0, \tau) \times \Omega, \\
D_{t} v(t, x)+C u(t, x)+D v(t, x)=f(t) z_{2}(x), \\
(t, x) \in(0, \tau) \times \Omega, \\
u(0, x)=u_{0}(x), \quad v(0, x)=v_{0}(x), \quad x \in \Omega, \\
D^{\tilde{\alpha}} u(t, x)=0, \quad|\widetilde{\alpha}| \leq m-1, \quad D^{\tilde{\delta}} v(t, x)=0, \\
\quad|\widetilde{\delta}| \leq r-1, \quad(t, x) \in(0, \tau) \times \partial \Omega, \\
\sigma u(t, \bar{x})+\mu v(t, \tilde{x})=g(t), \quad t \in(0, \tau),
\end{gathered}
$$

$\bar{x}$ and $\tilde{x}$ being two fixed elements in $\Omega$, admits a unique solution $((u, v), f) \in\left[C^{1+\theta}\left([0, \tau] ; C^{1+\omega}(\bar{\Omega}) \times C^{1+\omega}(\bar{\Omega})\right) \cap\right.$ $\left.C^{\theta}([0, \tau] ; \mathscr{D}(A) \times \mathscr{D}(D))\right] \times C^{\theta}([0, \tau] ; \mathbb{R})$ provided that the following conditions are satisfied:

(H1) $u_{0} \in \mathscr{D}(A), v_{0} \in \mathscr{D}(D), A u_{0}+B v_{0} \in \mathscr{D}(A), C u_{0}+$ $D v_{0} \in \mathscr{D}(D)$

$(\mathrm{H} 2) z_{1} \in \mathscr{D}(A), z_{2} \in \mathscr{D}(D), \sigma z_{1}(\bar{x})+\mu z_{2}(\tilde{x}) \neq 0$;

(H3) $g \in C^{1+\theta}([0, \tau] ; \mathbb{R}), 1-\beta<\theta<\beta, g(0)=\sigma u_{0}(\bar{x})+$ $\mu v_{0}(\tilde{x})$.

Notice that, if $p \leq 2 r-2$ and $q \leq 2 m-2$, then $\beta=\min \left\{\beta_{1}, \beta_{2}\right\}$ and condition $r q+m p+(1+\omega)(r+m)<4 m r$ is satisfied.

Problem 9. Here we solve an identification problem related to the Ornstein-Uhlenbeck operator in $\mathbb{R}^{n}, n \geq 1$. For this purpose, we refer to the monograph [11]. Such an operator is the prototype of an elliptic operator with unbounded coefficients and is defined on smooth functions $\varphi$ by

$$
\mathscr{A} \varphi(x)=\frac{1}{2} \operatorname{Tr}\left(Q D^{2} \varphi\right)(x)+\langle B x, D \varphi\rangle, \quad x \in \mathbb{R}^{n}
$$

where $D \varphi$ and $D^{2} \varphi$ denote, respectively, the gradient and the Hessian matrix of $\varphi$, while $Q$ and $B \neq O$ are $n \times n$ constant matrices, $Q$ being strictly positive definite and the spectrum $\sigma(B)$ of $B$ being contained in the left complex halfplane $\operatorname{Re} z<0$. 
It is well known that the realization $\mathscr{L}_{p}$ of $\mathscr{A}$ in the weighted space $L_{\mu}^{p}\left(\mathbb{R}^{n}\right)$, where

$$
\begin{aligned}
\mu(d x) & =(2 \pi)^{-n / 2}\left(\operatorname{det} Q_{\infty}\right)^{-1 / 2} e^{-\left\langle Q_{\infty}^{-1} x, x\right\rangle / 2} d x, \\
Q_{\infty} & =\int_{0}^{+\infty} e^{s B} Q e^{s B^{*}} d s,
\end{aligned}
$$

generates an analytic semigroup with domain $W_{\mu}^{2, p}\left(\mathbb{R}^{n}\right)$, where

$$
W_{\mu}^{k, p}\left(\mathbb{R}^{n}\right)=\left\{u \in L_{\mu}^{p}\left(\mathbb{R}^{n}\right): D^{\alpha} u \in L_{\mu}^{p}\left(\mathbb{R}^{n}\right),|\alpha| \leq k\right\}
$$

and is endowed with the norm $\|u\|_{W_{\mu}^{k, p}\left(\mathbb{R}^{n}\right)}=$ $\sum_{|\alpha| \leq k}\left\|D^{\alpha} u\right\|_{L_{\mu}^{p}\left(\mathbb{R}^{n}\right)}$, where $\|f\|_{L_{\mu}^{p}\left(\mathbb{R}^{n}\right)}=\left(\int_{\mathbb{R}^{n}}|f(x)|^{p} d \mu(x)\right)^{1 / p}$. Moreover, the spectrum of $\mathscr{L}_{p}$ is the discrete set

$$
\sigma\left(\mathscr{L}_{p}\right)=\left\{\lambda=\sum_{j=1}^{r} n_{j} \lambda_{j}: n_{j} \in \mathbb{N} \cup\{0\}, j=1, \ldots, r\right\},
$$

$\lambda_{1}, \ldots, \lambda_{r}$ denoting the (distinct) eigenvalues of $B$.

We note also that (cf. [11, Theorem 9.3.4])

$$
\begin{gathered}
\|\nabla u\|_{L_{\mu}^{p}\left(\mathbb{R}^{n}\right)} \leq C_{1}\|u\|_{L_{\mu}^{p}\left(\mathbb{R}^{n}\right)}^{1 / 2}\left\|\mathscr{L}_{p} u-u\right\|_{L_{\mu}^{p}\left(\mathbb{R}^{n}\right)}^{1 / 2}, \\
u \in W_{\mu}^{2, p}\left(\mathbb{R}^{n}\right), \\
\||x| u\|_{L_{\mu}^{p}\left(\mathbb{R}^{n}\right)} \leq C_{0}\|u\|_{W_{\mu}^{1, p}\left(\mathbb{R}^{n}\right)}, \quad u \in W_{\mu}^{2, p}\left(\mathbb{R}^{n}\right), \\
|x|=\left(\sum_{j=1}^{n} x_{j}^{2}\right)^{1 / 2} .
\end{gathered}
$$

Whence, since the operator $\mathscr{L}_{p}-1$ is continuously invertible, we easily deduce the estimates

$$
\begin{aligned}
& \||x| u\|_{L_{\mu}^{p}\left(\mathbb{R}^{n}\right)} \\
& \leq C_{0}\left[\|u\|_{L_{\mu}^{p}\left(\mathbb{R}^{n}\right)}+C_{1}\|u\|_{L_{\mu}^{p}\left(\mathbb{R}^{n}\right)}^{1 / 2}\left\|\mathscr{L}_{p} u-u\right\|_{L_{\mu}^{p}\left(\mathbb{R}^{n}\right)}^{1 / 2}\right] \\
& \leq C_{0}\left[C_{2}\|u\|_{L_{\mu}^{p}\left(\mathbb{R}^{n}\right)}^{1 / 2}\left\|\mathscr{L}_{p} u-u\right\|_{L_{\mu}^{p}\left(\mathbb{R}^{n}\right)}^{1 / 2}\right. \\
& \left.\quad+C_{1}\|u\|_{L_{\mu}^{p}\left(\mathbb{R}^{n}\right)}^{1 / 2}\left\|\mathscr{L}_{p} u-u\right\|_{L_{\mu}^{p}\left(\mathbb{R}^{n}\right)}^{1 / 2}\right] \\
& =C_{3}\|u\|_{L_{\mu}^{p}\left(\mathbb{R}^{n}\right)}^{1 / 2}\left\|\mathscr{L}_{p} u-u\right\|_{L_{\mu}^{p}\left(\mathbb{R}^{n}\right)}^{1 / 2}, \quad u \in W_{\mu}^{2, p}\left(\mathbb{R}^{n}\right) .
\end{aligned}
$$

Likewise we get the estimates

$$
\begin{aligned}
& \sum_{i, j=1}^{n}\left\|x_{i} D_{x_{j}} u\right\|_{L_{\mu}^{p}\left(\mathbb{R}^{n}\right)} \\
& \quad \leq C_{4}\left[\|\nabla u\|_{L_{\mu}^{p}\left(\mathbb{R}^{n}\right)}+\left\|D^{2} u\right\|_{L_{\mu}^{p}\left(\mathbb{R}^{n}\right)}\right] \\
& \quad \leq C_{5}\left\|\mathscr{L}_{p} u-u\right\|_{L_{\mu}^{p}\left(\mathbb{R}^{n}\right)}, \quad u \in W_{\mu}^{2, p}\left(\mathbb{R}^{n}\right) .
\end{aligned}
$$

Finally, recall that the dual space to $L_{\mu}^{p}\left(\mathbb{R}^{n}\right)$ can be identified with $L_{\mu}^{q}\left(\mathbb{R}^{n}\right), 1 / p+1 / q=1$.

Consider now the linear differential operator $B$ defined by

$$
B u=\sum_{j=1}^{n} a_{j}(x) D_{x_{j}} u+\sum_{j=1}^{n} b_{j}(x) x_{j} u, \quad u \in W_{\mu}^{1, p}\left(\mathbb{R}^{n}\right),
$$

where $a_{j}, b_{j}$ are uniformly continuous and bounded functions in $\mathbb{R}^{n}$.

The previous estimates yield

$$
\|B u\|_{L_{\mu}^{p}\left(\mathbb{R}^{n}\right)} \leq C_{7}\|u\|_{L_{\mu}^{p}\left(\mathbb{R}^{n}\right)}^{1 / 2}\left\|\mathscr{L}_{p} u-u\right\|_{L_{\mu}^{p}\left(\mathbb{R}^{n}\right)}^{1 / 2}, \quad \lambda \in \rho\left(\mathscr{L}_{p}\right) .
$$

Consider then the linear operator

$$
B_{1} u=\sum_{i, j=1}^{n} a_{i, j}(x) x_{i} D_{x_{j}} u, \quad u \in W_{\mu}^{1, p}\left(\mathbb{R}^{n}\right),
$$

where $a_{i, j}$ are uniformly continuous and bounded functions in $\mathbb{R}^{n}$

Reasoning as above, we conclude that $B_{1}$ satisfies the estimate

$$
\left\|B_{1}\left(\mathscr{L}_{p}-\lambda\right)^{-1} f\right\|_{L_{\mu}^{p}\left(\mathbb{R}^{n}\right)} \leq C_{8}\|f\|_{L_{\mu}^{p}\left(\mathbb{R}^{n}\right)}, \quad \lambda \in \rho\left(\mathscr{L}_{p}\right) .
$$

Consider now the identification problem:

$$
\begin{aligned}
& D_{t} u(t, x)=\left(\mathscr{L}_{p}-\lambda_{0}\right) u(t, x)+\sum_{j=1}^{n} a_{j}(x) D_{x_{j}} v(t, x) \\
&+\sum_{j=1}^{n} b_{j}(x) x_{j} v(t, x)+f(t) z_{1}(x), \\
&(t, x) \in(0, \tau] \times \mathbb{R}^{n}, \\
& D_{t} v(t, x)=\sum_{j=1}^{n} \bar{a}_{j}(x) D_{x_{j}} u(t, x)+\sum_{j=1}^{n} \bar{b}_{j}(x) x_{j} u(t, x) \\
&+\left(\mathscr{L}_{p}-\lambda_{0}\right) v(t, x)+f(t) z_{2}(x), \\
&(t, x) \in(0, \tau] \times \mathbb{R}^{n}, \\
& u(0, x)=u_{0}(x), \quad v(0, x)=v_{0}(x), \quad x \in \mathbb{R}^{n}, \\
& \int_{\mathbb{R}^{n}}\left\{\eta_{1}(x) u(t, x)+\eta_{2}(x) v(t, x)\right\} d \mu(x)=g(t), \\
& t \in[0, \tau],
\end{aligned}
$$

where $\eta_{1}, \eta_{2} \in L^{q}\left(\mathbb{R}^{n}\right), 1 / p+1 / q=1, g \in C^{1+\theta}([0, \tau] ; \mathbb{R})$, $\theta \in(0,1)$, and

$$
g(0)=\int_{\mathbb{R}^{n}}\left\{\eta_{1}(x) u_{0}(x)+\eta_{2}(x) v_{0}(x)\right\} \mu(d x) .
$$

Theorem 3 applies with $X=L_{\mu}^{p}\left(\mathbb{R}^{n}\right), p \in(1,+\infty)$. Here $\alpha=$ $1, \beta_{1}=\beta_{2}=1, \sigma_{1}=\sigma_{2}=1 / 2$, so that $\beta=1$. Assume 
further that $z_{1}, z_{2}, u_{0}, v_{0} \in W_{\mu}^{2, p}\left(\mathbb{R}^{n}\right)$ possess the additional properties:

$$
\begin{gathered}
\left(\mathscr{L}_{p}-\lambda_{0}\right) u_{0}+\sum_{j} a_{j}(x) D_{x_{j}} v_{0} \\
+\sum_{j} b_{j}(x) x_{j} v_{0} \in W_{\mu}^{2, p}\left(\mathbb{R}^{n}\right), \\
\sum_{j} \bar{a}_{j}(x) D_{x_{j}} u_{0}+\sum_{j} \bar{b}_{j}(x) x_{j} u_{0} \\
+\left(\mathscr{L}_{p}-\lambda_{0}\right) v_{0} \in W_{\mu}^{2, p}\left(\mathbb{R}^{n}\right), \\
\int_{\mathbb{R}^{n}}\left\{\eta_{1}(x) z_{1}(x)+\eta_{2}(x) z_{2}(x)\right\} d \mu(x) \neq 0, \quad t \in[0, \tau] .
\end{gathered}
$$

Then the identification Problems (66)-(321) admit a unique strict global solution $(u, v, f)$ with $u, v \in C^{1+\theta}([0, \tau]$; $\left.L_{\mu}^{p}\left(\mathbb{R}^{n}\right)\right) \cap C^{\theta}\left([0, \tau] ; W_{\mu}^{2, p}\left(\mathbb{R}^{n}\right)\right), f \in C^{\theta}([0, \tau] ; \mathbb{R})$.

We note that a corresponding result still holds if either of the lower order operators is replaced with $B_{1} u=$ $\sum_{i, j=1}^{n} a_{i, j}(x) x_{i} D_{x_{j}} u$.

Since $\mathbb{R}^{n}$ is an unbounded domain of cone type, the real interpolation spaces $\left(L_{\mu}^{p}\left(\mathbb{R}^{n}\right), W_{\mu}^{2, p}\left(\mathbb{R}^{n}\right)\right)_{\theta, p}, \theta \in(0,1)$ are well characterized. Exactly we have (cf. [9, Theorem 3.3.1]):

$$
\left(L_{\mu}^{p}\left(\mathbb{R}^{n}\right), W_{\mu}^{2, p}\left(\mathbb{R}^{n}\right)\right)_{\theta, \infty}=B_{p, \infty}^{2 \theta}\left(\mathbb{R}^{n} ; \mu\right) .
$$

Assume now that our data $\left(u_{0}, v_{0}, z_{1}, z_{2}\right)$ possess the following properties:

$$
\begin{gathered}
g \in C^{1+\theta}([0, \tau] ; \mathbb{R}), \quad 0<\theta<\theta_{0}<1, \\
u_{0}, v_{0} \in W_{\mu}^{2, p}\left(\mathbb{R}^{n}\right), \\
\left(\mathscr{L}_{p}-\lambda_{0}\right) u_{0}+\sum_{j=1}^{n} a_{j}(x) D_{x_{j}} v_{0} \\
+\sum_{j=1}^{n} b_{j}(x) x_{j} v_{0} \in B_{p, \infty}^{2 \theta}\left(\mathbb{R}^{n} ; \mu\right), \\
\sum_{j=1}^{n} \bar{a}_{j}(x) D_{x_{j}} u_{0}+\sum_{j=1}^{n} \bar{b}_{j}(x) x_{j} u_{0} \\
+\left(\mathscr{L}_{p}-\lambda_{0}\right) v_{0} \in B_{p, \infty}^{2 \theta}\left(\mathbb{R}^{n} ; \mu\right), \\
g(0)=\int_{\mathbb{R}^{n}}\left\{\eta_{1}(x) z_{1}(x)+\eta_{2}(x) z_{2}(x)\right\} d \mu(x) \neq 0, \\
z_{1}, z_{2} \in B_{p, \infty}^{2 \theta_{0}}\left(\mathbb{R}^{n} ; \mu\right) .
\end{gathered}
$$

Therefore, we can apply Theorem 3 or Proposition 5.1 in [1] and deduce the same conclusion as above.

We can analogously deal with the case when either of the lower order operators is replaced with $B_{1} u=$ $\sum_{i, j} a_{i, j}(x) x_{j} D_{x_{j}} u$.
Problem 10. In [12] the following remarkable result is proved. Let $X$ be a Hilbert space, let $\mathscr{A}: \mathscr{D}(\mathscr{A})$ be a densely defined, strictly positive self-adjoint operator, and let $M=\left[m_{i, j}\right]_{i, j=1}^{n}$ be a constant complex-valued matrix. Then the operator $A$, defined by

$$
\mathscr{D}(A)=\mathscr{D}(\mathscr{A})^{n}, \quad A=\mathscr{A} M=\left[\begin{array}{l}
m_{n, 1} \mathscr{A}, \ldots, m_{n, n} \mathscr{A} \\
m_{n, 1} \mathscr{A}, \ldots, m_{n, n} \mathscr{A}
\end{array}\right],
$$

generates a strongly continuous analytical semigroup in $H=$ $X^{n}$ if and only if (cf. [12, page 311]) the eigenvalues $\lambda_{i}$ of the matrix $M$ satisfy (i) $\operatorname{Re} \lambda_{i}<0$ if $\lambda_{i}$ is complex and (ii) $\lambda_{i} \leq 0$ if $\lambda_{i}$ is real.

This result allows to deal with a lot of very important problems related to evolution PDE's. We confine ourselves to describe an identification problem related to one of such models.

Suppose that $A=\mathscr{A} M$ generates an analytical semigroup in $H=X^{n}$. Then owing to [1, Theorem 2.1, page 45] (as improved in a following paper for Al Horani and Favini) the identification problem

$$
\begin{gathered}
y^{\prime}(t)-A y(t)=f(t) z, \quad 0 \leq t \leq \tau, \\
y(0)=y_{0}, \\
\Phi[y(t)]=g(t), \quad 0 \leq t \leq \tau,
\end{gathered}
$$

admits a unique global solution $(y, f) \in\left[C^{\theta}([0, \tau] ; \mathscr{D}(A)) \cap\right.$ $\left.C^{1+\theta}([0, \tau] ; X)\right] \times C^{\theta}([0, \tau] ; \mathbb{R})$, provided that

$$
\begin{gathered}
y_{0} \in \mathscr{D}(A), \quad A y_{0} \in \mathscr{D}_{A}(\theta, \infty)=\mathscr{D}_{\mathscr{A}}(\theta, \infty), \\
g \in C^{1+\theta}([0, \tau] ; \mathbb{R}), \quad \Phi\left[y_{0}\right]=g(0) \quad z \in \mathscr{D}_{A}(\theta, \infty) .
\end{gathered}
$$

Let us apply this result to the following thermoelastic PDE problem with simplified "hinged" homogeneous boundary conditions and Dirichlet thermal boundary conditions (cf. [12, page 317]), where the reference bounded domain $\Omega$ has a smooth boundary $\partial \Omega$ : determine a triplet of functions $w, v$ : $(0, \tau) \times \Omega \rightarrow \mathbb{R}$ and $f:[0, \tau] \rightarrow \mathbb{R}$ such that

$$
\begin{aligned}
& D_{t}^{2} w(t, x)+\Delta^{2} w(t, x)+\alpha \Delta v(t, x)=f(t) z_{1}(x), \\
& (t, x) \in Q \text {, } \\
& D_{t} v(t, x)-\eta \Delta v(t, x)-\alpha \Delta D_{t} w(t, x)=f(t) z_{2}(x), \\
& (t, x) \in Q \text {, } \\
& w(0, x)=w_{0}(x), \quad D_{t} w(0, x)=w_{1}(x), \quad v(0, x)=v_{0}(x), \\
& x \in \Omega \text {, }
\end{aligned}
$$

$$
w(t, x)=0, \quad \Delta w(t, x)=0, \quad v(t, x)=0,
$$

$$
(t, x) \in(0, \tau) \times \partial \Omega,
$$

$$
\begin{array}{r}
\left\langle\eta_{1}, w(t, \cdot)\right\rangle+\int_{\Omega} \eta_{2}(x) v(t, x) d x=g(t), \\
t \in(0, \tau),
\end{array}
$$


where $Q:=(0, \tau) \times \Omega$ and $\langle\cdot, \cdot\rangle$ denotes the duality pairing between $H^{2}(\Omega) \cap H_{0}^{1}(\Omega)$ and its dual space.

Introduce now the positive self-adjoint operator $\mathscr{A}$ defined by

$$
\mathscr{D}(\mathscr{A})=H^{2}(\Omega) \cap H_{0}^{1}(\Omega) \subset X=L^{2}(\Omega), \quad \mathscr{A}=-\Delta .
$$

This definition implies

$$
\mathscr{D}\left(\mathscr{A}^{2}\right)=\left\{h \in H^{4}(\Omega):\left.u\right|_{\partial \Omega}=\left.\Delta u\right|_{\partial \Omega}=0\right\}, \quad \mathscr{A}=\Delta^{2} .
$$

The previous differential equations read now

$$
\begin{aligned}
& D_{t}^{2} w(t, x)+\mathscr{A}^{2} w(t, x)-\alpha \mathscr{A} v(t, x)=f(t) z_{1}(x), \\
& (t, x) \in Q:=(0, \tau) \times \Omega, \\
& D_{t} v(t, x)+\eta \mathscr{A} v(t, x)+\alpha \mathscr{A} D_{t} w(t, x) \\
& =f(t) z_{2}(x), \quad(t, x) \in Q .
\end{aligned}
$$

Introduce then the Banach space $Y$, the new variable $u$, the vector $y$, and the operator $A_{l}$ defined by

$$
\begin{gathered}
Y=\mathscr{D}(\mathscr{A}) \times L^{2}(\Omega) \times L^{2}(\Omega), \quad u=D_{t} w, \\
y=(w, u, v)^{T}, \\
\mathscr{D}\left(A_{e}\right)=\mathscr{D}\left(\mathscr{A}^{2}\right) \times \mathscr{D}(\mathscr{A}) \times \mathscr{D}(\mathscr{A}), \\
A_{e}=\left[\begin{array}{ccc}
O & I & O \\
-\mathscr{A}^{2} & O & \alpha \mathscr{A} \\
O & -\alpha \mathscr{A} & -\eta \mathscr{A}
\end{array}\right] .
\end{gathered}
$$

Then system $(*)$ can be rewritten as the single equation:

$$
y^{\prime}(t)-\mathscr{A}_{e} y(t)=f(t) z, \quad 0 \leq t \leq \tau .
$$

In [12, page 318] it is shown that operator $A_{e}$ generates an analytic semigroup on $Y$ if and only if the operator

$$
A=\left[\begin{array}{ccc}
O & \mathscr{A} & O \\
-\mathscr{A} & O & \alpha \mathscr{A} \\
O & -\alpha \mathscr{A} & -\eta \mathscr{A}
\end{array}\right]
$$

generates a strongly continuous analytic semigroup in the space $H=L^{2}(\Omega)^{3}$.

Indeed, in this case we have $A=\mathscr{A} M$, where the constant matrix $M$ is defined by

$$
M=\left[\begin{array}{ccc}
0 & 1 & 0 \\
-1 & 0 & \alpha \\
0 & -\alpha & -\eta
\end{array}\right] .
$$

Consider then the eigenvalue equation $m(\lambda)=: \operatorname{det}(\lambda I-M)=$ $\lambda^{3}+\eta \lambda^{2}+\left(\alpha^{2}+1\right) \lambda+\eta=0$ and observe that (i) since $m(-\eta)=-\alpha^{2} \eta<0$ and $m(0)=\eta>0$, the matrix $M$ admits a real negative eigenvalue $\lambda_{1}=-a(\alpha, \eta) \in(-\eta, 0)$; (ii) all the real eigenvalues of $M$ are strictly negative; (iii) all the nonreal eigenvalues $\lambda$ have negative real parts $\operatorname{Re} \lambda=[a(\alpha, \eta)-\eta] / 2$, since the sum of is $-\eta$. Consequently, all the eigenvalues of $M$ have negative real parts so that $A$ generates an analytic semigroup in $H$ (resp., $Y$ ).

Let us consider first our identification problem in $Y$. Since

$$
\begin{gathered}
z=\left[0, z_{1}, z_{2}\right]^{T}, \\
\Phi[y]=\int_{\Omega}\left[\eta_{1}(x) y_{1}(x)+\eta_{2}(x) y_{3}(x)\right] d x, \\
\eta_{1}, \eta_{2} \in L^{2}(\Omega)
\end{gathered}
$$

we must require

$$
\Phi[z]=\int_{\Omega} \eta_{2}(x) z_{2}(x) d x \neq 0 .
$$

Moreover, we assume

$$
\begin{gathered}
g \in C^{1+\theta}([0, \tau] ; \mathbb{R}), \\
g(0)=\int_{\Omega}\left[\eta_{1}(x) w_{0}(x)+\eta_{2}(x) v_{0}(x)\right] d x, \quad\left(w_{0}, w_{1}, v_{0}\right) \in Y, \\
{\left[\begin{array}{ccc}
O & I & O \\
-\mathscr{A}^{2} & O & \alpha \mathscr{A} \\
O & -\alpha \mathscr{A} & -\eta \mathscr{A}
\end{array}\right]\left[\begin{array}{c}
w_{0} \\
w_{1} \\
v_{0}
\end{array}\right]} \\
=\left[\begin{array}{cc}
w_{1} \\
-\mathscr{A}^{2} w_{0}+\alpha \mathscr{A} v_{0} \\
-\alpha \mathscr{A}_{1}-\eta \mathscr{A} v_{0}
\end{array}\right] \in\left(Y ; \mathscr{D}\left(A_{l}\right)\right)_{\theta, \infty} \\
=\left(\mathscr{D}(\mathscr{A}) \times L^{2}(\Omega) \times L^{2}(\Omega) ;\right. \\
\left.\quad \mathscr{D}\left(\mathscr{A}^{2}\right) \times \mathscr{D}(\mathscr{A}) \times \mathscr{D}(\mathscr{A})\right)_{\theta, \infty} \\
=\left(\mathscr{D}(\mathscr{A}) ; \mathscr{D}\left(\mathscr{A}^{2}\right)\right)_{\theta, \infty} \times\left(L^{2}(\Omega) ; \mathscr{D}(\mathscr{A})\right)_{\theta, \infty} \\
\quad \times\left(L^{2}(\Omega) ; \mathscr{D}(\mathscr{A})\right)_{\theta, \infty}, \\
\left(0, z_{1}, z_{2}\right) \in\left(\mathscr{D}(\mathscr{A}) ; \mathscr{D}\left(\mathscr{A}^{2}\right)\right)_{\theta_{0}, \infty} \\
\quad \times\left(L^{2}(\Omega) ; \mathscr{D}(\mathscr{A})\right)_{\theta_{0}, \infty} \times\left(L^{2}(\Omega) ; \mathscr{D}(\mathscr{A})\right)_{\theta_{0}, \infty} \\
\Longleftrightarrow z_{1}, z_{2} \in\left(L^{2}(\Omega) ; \mathscr{D}(A)\right)_{\theta_{0}, \infty} .
\end{gathered}
$$

Note that the previous spaces are well characterised as Besov spaces (cf. [9]). More precisely, since $\mathscr{A}$ is a positive operator we have (cf. [9, page 105])

$$
\left(\mathscr{D}(\mathscr{A}) ; \mathscr{D}\left(\mathscr{A}^{2}\right)\right)_{\theta, \infty}=\left(L^{2}(\Omega) ; \mathscr{D}\left(\mathscr{A}^{2}\right)\right)_{(1+\theta) / 2, \infty} \text {. }
$$

On the other hand, if $\Lambda: \mathscr{D}(\mathscr{A}) \subset X \rightarrow X$ is a positive operator, from [9, Theorem 1.14.3(b)] it follows that $\left(X, \mathscr{D}\left(\Lambda^{2}\right)\right)_{\sigma, \infty}, \sigma \in(1 / 2,1)$, coincides with $\{x \in \mathscr{D}(\mathscr{A})$ : 
$\left.\left\|t^{2 \sigma-1}\left[\Lambda(\Lambda+1)^{-1}\right] \Lambda x\right\|_{L_{\infty}^{*}(X)}\right\}<\infty=\{x \in \mathscr{D}(\Lambda): \Lambda x \in$ $\left.(X, D(\Lambda))_{2 \sigma-1, \infty}\right\}$. Therefore,

$$
\begin{aligned}
& \left(L^{2}(\Omega), \mathscr{D}\left(\mathscr{A}^{2}\right)\right)_{(1+\theta) / 2, \infty} \\
& \quad=\left\{u \in \mathscr{D}(\mathscr{A}): \mathscr{A} u \in\left(L^{2}(\Omega), \mathscr{D}(\mathscr{A})\right)_{\theta, \infty}\right\} .
\end{aligned}
$$

Using now the notation in [9, page 321],

$$
\mathscr{D}(\mathscr{A})=H_{p, B_{1}}^{2}, \quad B_{1} u=\left.u\right|_{\partial \Omega},
$$

we deduce the following characterization in terms of Besov spaces:

$$
\begin{aligned}
& \left(L^{2}(\Omega), \mathscr{D}(\mathscr{A})\right)_{\theta, \infty} \\
& =B_{2, \infty, B_{1}}^{2 \theta}(\Omega), \quad \text { if } \theta \neq \frac{1}{4}, \\
& =\left\{f \in B_{2,2, B_{1}}^{1 / 2}(\Omega): B_{1} f=\left.f\right|_{\partial \Omega}, \in B_{2,2, B_{1}}^{1 / 2}(\Omega)\right\}, \quad \text { if } \theta=\frac{1}{4},
\end{aligned}
$$

cf. [9, Definition 4.3.2, page 317].

Therefore, we assume that our data satisfy the following properties:

$$
\begin{gathered}
\left(w_{0}, w_{1}, v_{0}\right) \in \mathscr{D}\left(\mathscr{A}^{2}\right) \times \mathscr{D}(\mathscr{A}) \times \mathscr{D}(\mathscr{A}), \\
\mathscr{A} w_{1},-\mathscr{A}^{2} w_{0}+\alpha \mathscr{A} v_{0},-\alpha \mathscr{A} w_{1} \\
-\eta \mathscr{A} v_{0} \in\left(L^{2}(\Omega), \mathscr{D}(\mathscr{A})\right)_{\theta, \infty} \\
\Longleftrightarrow \mathscr{A}^{2} w_{0}, \mathscr{A} w_{1}, \mathscr{A} v_{0} \in\left(L^{2}(\Omega), \mathscr{D}(\mathscr{A})\right)_{\theta, \infty}, \\
z_{1}, z_{2} \in\left(L^{2}(\Omega), \mathscr{D}(\mathscr{A})\right)_{\theta_{0}, \infty}, \quad 0<\theta<\theta_{0}<1, \\
\int_{\Omega} \eta_{2}(x) z_{2}(x) d x \neq 0, \\
g(0)=\int_{\Omega}\left[\eta_{1}(x) w_{0}(x)+\eta_{2}(x) v_{0}(x)\right] d x .
\end{gathered}
$$

Under these assumptions the identification Problem (P) admits a unique solution $(w, v, f)$ such that $w \in\left[C^{2+\theta}([0, \tau]\right.$; $\left.\left.L^{2}(\Omega)\right) \times C^{1+\theta}([0, \tau] ; \mathscr{D}(\mathscr{A})) \times C^{\theta}\left([0, \tau] ; \mathscr{D}\left(\mathscr{A}^{2}\right)\right)\right], v \in$ $\left[C^{1+\theta}\left([0, \tau] ; L^{2}(\Omega)\right) \times C^{\theta}([0, \tau] ; \mathscr{D}(\mathscr{A}))\right]$ and $f \in C^{\theta}([0$, $\tau] ; \mathbb{R})$.

Remark 11. Since $\mathscr{A}$ is a strictly positive and self-adjoint operator in the Hilbert space $X$, we could treat in the same way the case when $X=L_{\mu}^{2}\left(\mathbb{R}^{N}\right)$, as in Problem 9. Indeed, the realization is that $\mathscr{A}$ of $-\mathscr{L}_{2}+I$, with $B Q=Q B^{*}, B=-Q_{\infty}^{-1}$ is strictly positive and self-adjoint [11, Proposition 9.3.10, page 251].

Remark 12. If we consider the identification Problem (P) with the additional information

$$
\int_{\Omega} \eta_{3}(x) D_{t} w(t, x) d x=g(t), \quad t \in[0, \tau],
$$

then, setting

$$
\Phi[y]=\left(0, \int_{\Omega} \eta_{3}(x) y_{2}(x) d x, 0\right)
$$

under the assumptions

$$
\begin{gathered}
\int_{\Omega} \eta_{3}(x) z_{1}(x) d x \neq 0, \\
\left(z_{1}, z_{2}\right) \in\left(L^{2}(\Omega), \mathscr{D}(\mathscr{A} \mathscr{A})\right)_{\theta_{0}, \infty}, \\
g \in C^{1+\theta}([0, \tau] ; \mathbb{R}), \quad g(0)=\int_{\Omega} \eta_{3}(x) w_{1}(x) d x, \\
\eta_{3} \in L^{2}(\Omega), \\
\mathscr{A}^{2} w_{0}, \mathscr{A} w_{1}, \mathscr{A} v_{0} \in\left(L^{2}(\Omega), \mathscr{D}(\mathscr{A})\right)_{\theta, \infty} .
\end{gathered}
$$

Problem (P), admits a unique solution $(w, v, f)$.

\section{First-Order Systems of Singular Differential Equations in Banach Spaces and Identification Problems}

Here we face identification problems for systems of singular first-order differential equations in the Banach space $X \times X$, both applying the general results described in Section 5 and developing adhoc methods in order to improve the corresponding consequences in some cases. For this purpose, we need some preliminary lemmas on the resolvent estimates.

Theorem 13. Suppose that the closed linear operators $L_{1}, L_{2}$, $L_{4}, M_{1}, M_{2}, M_{4}$ in $X$ satisfy the conditions:

$$
\begin{aligned}
& \left\|M_{1}\left(z M_{1}+L_{1}\right)^{-1}\right\|_{\mathscr{L}(X)} \leq C(1+|z|)^{-\beta_{1}}, \quad \forall z \in \Sigma_{\alpha}, \\
& \left\|M_{4}\left(z M_{4}+L_{4}\right)^{-1}\right\|_{\mathscr{L}(X)} \leq C(1+|z|)^{-\beta_{4}}, \quad \forall z \in \Sigma_{\alpha}, \\
& \left\|M_{2} x\right\| \leq C\left\|M_{4} x\right\|^{\delta}\|x\|^{1-\delta}, \quad 0<\delta \leq 1, \quad x \in D\left(M_{4}\right),
\end{aligned}
$$

$$
\mathscr{D}\left(L_{4}\right) \subseteq \mathscr{D}\left(L_{2}\right) \text { continuously. }
$$

Then the matrix operators $A$ and $B$ defined by

$$
A=\left[\begin{array}{cc}
L_{1} & L_{2} \\
0 & L_{4}
\end{array}\right], \quad B=\left[\begin{array}{cc}
M_{1} & M_{2} \\
0 & M_{4}
\end{array}\right]
$$

with $\mathscr{D}(A)=\mathscr{D}\left(L_{1}\right) \times \mathscr{D}\left(L_{4}\right), \mathscr{D}(B)=\mathscr{D}\left(M_{1}\right) \times D\left(M_{4}\right)$, satisfy the estimate

$$
\left\|B(z B+A)^{-1}\right\|_{\mathscr{L}(X \times X)} \leq C(1+|z|)^{-\beta}
$$

$\forall z \in \Sigma_{\alpha} \cap \overline{B(0, R)}^{c}$, for large enough $R$,

where $\beta=\beta_{1}+\beta_{4}+\delta-2$. 
Proof. First of all, we need to verify that $A$ and $B$ are closed linear operators.

Let $x_{n} \in \mathscr{D}\left(M_{1}\right), y_{n} \in D\left(M_{4}\right), x_{n} \rightarrow x, y_{n} \rightarrow y$, $M_{1} x_{n}+M_{2} y_{n} \rightarrow \xi, M_{4} y_{n} \rightarrow \eta$ as $n \rightarrow+\infty$. We immediately deduce that $y \in D\left(M_{4}\right)$ ad $M_{4} y=\eta$. Moreover,

$$
\left\|M_{2} y_{n}-M_{2} y_{m}\right\| \leq C\left\|M_{4} y_{m}-M_{4} y_{m}\right\|^{\delta}\left\|y_{n}-y_{m}\right\|^{1-\delta}
$$

implies that $M_{2} y_{n} \rightarrow \sigma$ as $n \rightarrow+\infty$ for some $\sigma \in X$. Since $M_{2}$ is closed $\delta=M_{2} y$. But $M_{1}$ is closed too, so that $x \in \mathscr{D}\left(M_{1}\right)$ and $M_{1} x+M_{2} y=\xi$. Analogously, if $x_{n} \in$ $\mathscr{D}\left(L_{1}\right), y_{n} \in \mathscr{D}\left(L_{4}\right), x_{n} \rightarrow x, y_{n} \rightarrow y$ as $n \rightarrow+\infty$,
$L_{1} x_{n}+L_{2} y_{n} \rightarrow \xi, L_{4} y_{n} \rightarrow \eta$, then $y \in \mathscr{D}\left(L_{4}\right)$ and $L_{4} y=\eta$. Since $\mathscr{D}\left(L_{4}\right) \subseteq \mathscr{D}\left(L_{2}\right)$

$$
\begin{aligned}
& \left\|y_{n}-y_{m}\right\|+\left\|L_{2} y_{n}-L_{2} y_{m}\right\| \\
& \quad \leq C\left[\left\|y_{n}-y_{m}\right\|+\left\|L_{4} y_{n}-L_{4} y_{m}\right\|\right]
\end{aligned}
$$

and thus $L_{2} y_{n}$ has a limit $\sigma$ as $n \rightarrow+\infty$. It follows that $L_{2} y_{n}$ tends to $\sigma=L_{2} y$ so that we can conclude as the above.

We easily see that

$$
\begin{aligned}
& (z B+A)^{-1} \\
& =\left[\begin{array}{cc}
\left(z M_{1}+L_{1}\right)^{-1} & -\left(z M_{1}+L_{1}\right)^{-1}\left(z M_{2}+L_{2}\right)\left(z M_{4}+L_{4}\right)^{-1} \\
0 & \left(z M_{4}+L_{4}\right)^{-1}
\end{array}\right]
\end{aligned}
$$

so that

$$
B(z B+A)^{-1}=\left[\begin{array}{cc}
M_{1}\left(z M_{1}+L_{1}\right)^{-1} & -M_{1}\left(z M_{1}+L_{1}\right)^{-1}\left(z M_{2}+L_{2}\right)\left(z M_{4}+L_{4}\right)^{-1}+M_{2}\left(z M_{4}+L_{4}\right)^{-1} \\
0 & M_{4}\left(z M_{4}+L_{4}\right)^{-1}
\end{array}\right] .
$$

Notice that if $z \neq 0$, then

$$
\begin{aligned}
- & M_{1}\left(z M_{1}+L_{1}\right)^{-1}\left(z M_{2}+L_{2}\right)\left(z M_{4}+L_{4}\right)^{-1} \\
& +M_{2}\left(z M_{4}+L_{4}\right)^{-1} \\
= & \frac{-1}{z}\left[I-L_{1}\left(z M_{1}+L_{1}\right)^{-1}\right] \\
& \times\left(z M_{2}+L_{2}\right)\left(z M_{4}+L_{4}\right)^{-1}+M_{2}\left(z M_{4}+L_{4}\right)^{-1} \\
= & -\left[I-L_{1}\left(z M_{1}+L_{1}\right)^{-1}\right] M_{2}\left(z M_{4}+L_{4}\right)^{-1} \\
& -\frac{1}{z}\left[I-L_{1}\left(z M_{1}+L_{1}\right)^{-1}\right] L_{2}\left(z M_{4}+L_{4}\right)^{-1} \\
& +M_{2}\left(z M_{4}+L_{4}\right)^{-1} \\
= & L_{1}\left(z M_{1}+L_{1}\right)^{-1} M_{2}\left(z M_{4}+L_{4}\right)^{-1} \\
& -z^{-1}\left[I-L_{1}\left(z M_{1}+L_{1}\right)^{-1}\right] L_{2}\left(z M_{4}+L_{4}\right)^{-1} .
\end{aligned}
$$

Therefore, from (97)

$$
\begin{aligned}
& \left\|M_{2}\left(z M_{4}+L_{4}\right)^{-1}\right\|_{\mathscr{L}(X)} \\
& \quad \leq C\left\|M_{4}\left(z M_{4}+L_{4}\right)^{-1}\right\|_{\mathscr{L}(X)}^{\delta}\left\|\left(z M_{4}+L_{4}\right)^{-1}\right\|_{\mathscr{L}(X)}^{1-\delta} \\
& \quad \leq C(1+|z|)^{-\beta_{4} \delta}(1+|z|)^{\left(1-\beta_{4}\right)(1-\delta)} \\
& \quad \leq C(1+|z|)^{1-\beta_{4}-\delta},
\end{aligned}
$$

we deduce the bound

$$
\begin{aligned}
\| & -M_{1}\left(z M_{1}+L_{1}\right)^{-1}\left(z M_{2}+L_{2}\right)\left(z M_{4}+L_{4}\right)^{-1} \\
& +M_{2}\left(z M_{4}+L_{4}\right)^{-1} \|_{\mathscr{L}(X)} \\
& \leq c_{1}(1+|z|)^{2-\beta_{1}-\beta_{4}-\delta}+c_{2}(1+|z|)^{-\beta_{1}-\beta_{4}+1} \\
& \leq c(1+|z|)^{2-\beta_{1}-\beta_{4}-\delta} .
\end{aligned}
$$

Since $\beta_{1}+\beta_{4}+\delta-2 \leq \min \left\{\beta_{1}, \beta_{4}\right\}$, this completes the proof.

We can now apply Proposition 29 to the identification problem:

$$
\begin{gathered}
\frac{d}{d t}\left[\begin{array}{cc}
M_{1} & M_{2} \\
0 & M_{4}
\end{array}\right]\left[\begin{array}{l}
x(t) \\
y(t)
\end{array}\right] \\
+\left[\begin{array}{cc}
L_{1} & L_{2} \\
0 & L_{4}
\end{array}\right]\left[\begin{array}{l}
x(t) \\
y(t)
\end{array}\right]=f(t)\left[\begin{array}{l}
z_{1} \\
z_{2}
\end{array}\right], \quad \forall t \in[0, \tau], \\
\left(M_{1} x+M_{2} y\right)(0)=M_{1} x_{0}+M_{2} y_{0}, \\
\left(M_{4} y\right)(0)=M_{4} y_{0}, \\
\Phi_{1}\left[M_{1} x(t)+M_{2} y(t)\right]+\Phi_{2}\left[M_{4} y(t)\right]=g(t),
\end{gathered}
$$$$
\forall t \in[0, \tau] .
$$

Suppose that assumptions (95)-(98) are satisfied, with $\alpha+\beta_{1}+$ $\beta_{4}+\delta>7 / 2$ and let $4-\alpha-\beta_{1}-\beta_{4}-\delta<\theta<\alpha+\beta_{1}+\beta_{4}+\delta-3$. Further, assume

$$
\begin{gathered}
{\left[\begin{array}{c}
z_{1} \\
z_{2}
\end{array}\right]=\left[\begin{array}{c}
M_{1} z_{1}^{*}+M_{2} z_{2}^{*} \\
M_{4} z_{2}^{*}
\end{array}\right],} \\
{\left[\begin{array}{c}
L_{1} x_{0}+L_{2} y_{0} \\
L_{4} y_{0}
\end{array}\right]=\left[\begin{array}{c}
M_{1} x_{0}^{*}+M_{2} y_{0}^{*} \\
M_{4} y_{0}^{*}
\end{array}\right]}
\end{gathered}
$$


with

$$
\begin{array}{ll}
z_{1}^{*} \in \mathscr{D}\left(L_{1}\right), & z_{2}^{*} \in \mathscr{D}\left(L_{4}\right), \\
x_{0}^{*} \in \mathscr{D}\left(L_{1}\right), & y_{0}^{*} \in \mathscr{D}\left(L_{4}\right) .
\end{array}
$$

We have the following.

Theorem 14. Under assumptions (95)-(98), if $\alpha+\beta_{1}+\beta_{4}+\delta>$ $7 / 2, \theta \in\left(4-\alpha-\beta_{1}-\beta_{4}-\delta, \alpha+\beta_{1}+\beta_{4}+\delta-3\right)$, (109) and (110) hold together with $g \in C^{1+\theta}([0, \tau] ; \mathbb{R}), \Phi_{1}, \Phi_{2} \in X^{*}$,

$$
\begin{gathered}
\Phi_{1}\left[z_{1}\right]+\Phi_{2}\left[z_{2}\right] \neq 0, \\
\Phi_{1}\left[M_{1} x_{0}+M_{2} y_{0}\right]+\Phi_{2}\left[M_{4} y_{0}\right]=g(0),
\end{gathered}
$$

then the identification Problem (108) admits a unique global solution $(x, y, f) \in C^{\theta}\left([0, \tau] ; \mathscr{D}\left(L_{1}\right)\right) \times C^{\theta}\left([0, \tau] ; \mathscr{D}\left(L_{4}\right)\right) \times$ $C^{\theta}([0, \tau] ; \mathbb{R}), M_{1} x+M_{2} y \in C^{1+\theta}([0, \tau] ; X), M_{4} y \in C^{1+\theta}([0$, $\tau] ; X)$.

Remark 15. When $M_{2}=0$, we can take $\delta=1$ in (97).

Particular attention deserves the case when $\alpha=\beta_{1}=\beta_{4}=$ $\delta=1, X$ being a reflexive Banach space. Then

$$
\left\|B(z B+A)^{-1}\right\|_{\mathscr{L}(X \times X)} \leq C(1+|z|)^{-1}, \quad \forall z \in k_{0}+\Sigma_{1} .
$$

Let $\mathbb{T}=B\left(k_{0} B+A\right)^{-1}$; that is,

$$
\mathbb{T}:=\left[\begin{array}{cc}
M_{1}\left(k_{0} M_{1}+L_{1}\right)^{-1} & L_{1}\left(k_{0} M_{1}+L_{1}\right)^{-1} M_{2}\left(k_{0} M_{4}+L_{4}\right)^{-1} \\
& -k_{0}^{-1}\left[I-L_{1}\left(k_{0} M_{1}+L_{1}\right)^{-1}\right] L_{2}\left(k_{0} M_{4}+L_{4}\right)^{-1} \\
0 & M_{4}\left(k_{0} M_{4}+L_{4}\right)^{-1}
\end{array}\right] .
$$

Then $X \times X=N(\mathbb{T}) \oplus \overline{R(\mathbb{T})}$. Denote by $P$ the projection operator onto $N(\mathbb{T})$ along $\overline{R(\mathbb{T})}$ and suppose $0<\theta<\theta_{0}<$ $1, g \in C^{1+\theta}([0, \tau] ; \mathbb{R}), \Phi_{1}, \Phi_{2} \in X^{*},(112)$ holds, if $(I-$ $\mathbb{P})\left[z_{1}, z_{2}\right]^{T}=\left[\bar{z}_{1}, \bar{z}_{2}\right]^{T}$,

$$
\begin{gathered}
\Phi_{1}\left[\bar{z}_{1}\right]+\Phi_{2}\left[\bar{z}_{2}\right] \neq 0, \\
\sup _{t>0} t^{\theta} \|(t \mathbb{\mathbb { T }}+I)^{-1}(I-\mathbb{P}) \\
\times\left[\begin{array}{cc}
k_{0} M_{1}+L_{1} & k_{0} M_{2}+L_{2} \\
0 & k_{0} M_{4}+L_{4}
\end{array}\right]\left[\begin{array}{l}
x_{0} \\
y_{0}
\end{array}\right] \|_{\overline{R(\mathbb{T})}}<+\infty, \\
\sup _{t>0} t^{\theta_{0}}\left\|(t \mathbb{\mathbb { T }}+I)^{-1}\left[\begin{array}{l}
\bar{z}_{1} \\
\bar{z}_{2}
\end{array}\right]\right\|_{\frac{R(\mathbb{T})}{}<+\infty .}
\end{gathered}
$$

After applying Proposition 32, we get the following result. identification Problem (108) admits a unique global solution $(x, y, f) \in C^{\theta}\left([0, \tau] ; \mathscr{D}\left(L_{1}\right)\right) \times C^{\theta}\left([0, \tau] ; \mathscr{D}\left(L_{4}\right)\right) \times C^{\theta}([0, \tau] ;$ $\mathbb{R})$, such that $M_{1} x+M_{2} y \in C^{1+\theta}([0, \tau] ; X), M_{4} y \in C^{1+\theta}([0$, $\tau] ; X)$. Moreover, if $\mathbb{T}$ has a closed range, then (116)-(117) can be dropped out.

Next we extend Theorem 13 to nontriangular operator matrices. Precisely, we consider the system $(A, B)$ where

$$
A=\left[\begin{array}{ll}
L_{1} & L_{2} \\
L_{3} & L_{4}
\end{array}\right], \quad B=\left[\begin{array}{cc}
M_{1} & M_{2} \\
0 & M_{4}
\end{array}\right] .
$$

We have the following.

Lemma 17. Assume (95)-(98) hold and $\mathscr{D}\left(M_{1}\right) \subseteq D\left(L_{3}\right)$, with

$$
\left\|L_{3} x\right\| \leq C\left\|M_{1} x\right\|^{\delta_{1}}\|x\|^{1-\delta_{1}}, \quad x \in \mathscr{D}\left(M_{1}\right), 0<\delta_{1} \leq 1 .
$$

Proposition 16. Let $X$ be a reflexive Banach space, suppose (95)-(98) hold with $\alpha=\beta_{1}=\beta_{4}=\delta=1, \mathbb{T}, \mathbb{P}$ being as above, $g \in C^{1+\theta}([0, \tau] ; \mathbb{R}), 0<\theta<\theta_{0}<1,\left(x_{0}, y_{0}\right) \in$ $\mathscr{D}\left(L_{1}\right) \times \mathscr{D}\left(L_{4}\right), \Phi_{1}, \Phi_{2} \in X^{*}$. If (112)-(117) hold, then the
If $\beta_{1}+\beta_{4}+\delta+\delta_{1}>3$, then $\left\|B(z B+A)^{-1}\right\|_{\mathscr{L}(X \times X)} \leq C(1+$ $|z|)^{-\beta}, \forall z \in \Sigma_{\alpha},|z|$ large, when $\beta=\beta_{1}+\beta_{4}+\delta-2$. 
Proof. We proceed by a perturbation argument. Write

$$
\begin{aligned}
& {\left[\begin{array}{cc}
z M_{1}+L_{1} & z M_{2}+L_{2} \\
L_{3} & z M_{4}+L_{4}
\end{array}\right] } \\
&=\left[\begin{array}{ll}
0 & 0 \\
L_{3} & 0
\end{array}\right]+\left[\begin{array}{cc}
z M_{1}+L_{1} & z M_{2}+L_{2} \\
0 & z M_{4}+L_{4}
\end{array}\right] \\
&=\left(\left[\begin{array}{ll}
I & 0 \\
0 & I
\end{array}\right]+\left[\begin{array}{cc}
0 & 0 \\
L_{3} & 0
\end{array}\right] \times\left[\begin{array}{cc}
\left(z M_{1}+L_{1}\right)^{-1}-\left(z M_{1}+L_{1}\right)^{-1}\left(z M_{2}+L_{2}\right)\left(z M_{4}+L_{4}\right)^{-1} & \left(z M_{4}+L_{4}\right)^{-1} \\
0 & \left(z M_{1}\right.
\end{array}\right]\right) \\
&= \\
&\left.\times\left[\begin{array}{cc}
z M_{1}+L_{1} & z M_{2}+L_{2} \\
0 & z M_{4}+L_{4}
\end{array}\right]\right) \\
& \quad\left(\left[\begin{array}{cc}
I & 0 \\
0 & I
\end{array}\right]+\left[\begin{array}{cc}
z M_{1}+L_{1} & z M_{2}+L_{2} \\
L_{3}\left(z M_{1}+L_{1}\right)^{-1} & -L_{3}\left(z M_{1}+L_{1}\right)^{-1}\left(z M_{2}+L_{2}\right)\left(z M_{4}+L_{4}\right)^{-1}
\end{array}\right]\right) \\
& \times\left[\begin{array}{cc}
z M_{4}+L_{4}
\end{array}\right] .
\end{aligned}
$$

In view of (119), we get first

$$
\begin{aligned}
& \left\|L_{3}\left(z M_{1}+L_{1}\right)^{-1}\right\|_{\mathscr{L}(X)} \leq C(1+|z|)^{1-\beta_{1}-\delta_{1}}, \\
& \qquad\left(z M_{2}+L_{2}\right)\left(z M_{4}+L_{4}\right)^{-1} \|_{\mathscr{L}(X)} \text { (cf. Lemma 17) } \\
& \quad \leq|z|\left\|M_{2}\left(z M_{4}+L_{4}\right)^{-1}\right\|_{\mathscr{L}(X)}+\left\|L_{2}\left(z M_{4}+L_{4}\right)^{-1}\right\|_{\mathscr{L}(X)} \\
& \quad \leq C\left[(1+|z|)^{2-\beta_{4}-\delta}+(1+|z|)^{1-\beta_{4}}\right] \\
& \quad \leq C(1+|z|)^{2-\beta_{4}-\delta},
\end{aligned}
$$

and then

$$
\begin{aligned}
& \left\|L_{3}\left(z M_{1}+L_{1}\right)^{-1}\left(z M_{2}+L_{2}\right)\left(z M_{4}+L_{4}\right)^{-1}\right\| \\
& \quad \leq C(1+|z|)^{3-\beta_{1}-\beta_{4}-\delta-\delta_{1}} .
\end{aligned}
$$

Notice now that $1-\beta_{1}-\delta_{1} \leq 3-\beta_{1}-\beta_{4}-\delta-\delta_{1}$ implies that the linear operator

$\left[\begin{array}{ll}I & 0 \\ 0 & I\end{array}\right]$

$+\left[\begin{array}{cc}0 & 0 \\ L_{3}\left(z M_{1}+L_{1}\right)^{-1} & -L_{3}\left(z M_{1}+L_{1}\right)^{-1}\left(z M_{2}+L_{2}\right)\left(z M_{4}+L_{4}\right)^{-1}\end{array}\right]$

has a bounded inverse for $|z|$ large and its inverse can be estimated in the norm of $\mathscr{L}(X \times X)$ by $1 / 2$, for example. It follows that $B(z B+A)^{-1}$ has precisely the same bound as in Lemma 17.
Theorem 18. Under assumptions (95)-(98) and (119), if $\alpha+$ $\beta_{1}+\beta_{4}+\delta>7 / 2$ and $\beta_{1}+\beta_{4}+\delta+\delta_{1}>3, \theta \in\left(4-\alpha-\beta_{1}-\right.$ $\left.\beta_{4}-\delta, \alpha+\beta_{1}+\beta_{4}+\delta-3\right)$,

$$
\begin{gathered}
{\left[\begin{array}{c}
z_{1} \\
z_{2}
\end{array}\right]=\left[\begin{array}{c}
M_{1} z_{1}^{*}+M_{2} z_{2}^{*} \\
M_{4} z_{2}^{*}
\end{array}\right]} \\
{\left[\begin{array}{c}
L_{1} x_{0}+L_{2} y_{0} \\
L_{3} x_{0}+L_{4} y_{0}
\end{array}\right]=\left[\begin{array}{c}
M_{1} x_{0}^{*}+M_{2} y_{0}^{*} \\
M_{4} y_{0}^{*}
\end{array}\right]}
\end{gathered}
$$

for some $z_{1}^{*}, x_{0}^{*} \in \mathscr{D}\left(L_{1}\right), z_{2}^{*}, y_{0}^{*} \in \mathscr{D}\left(L_{4}\right), g \in C^{1+\theta}([0, \tau] ; \mathbb{R})$, $\Phi_{1}, \Phi_{2} \in X^{*}$ and (111)-(112) hold, then the identification problem

$$
\begin{array}{r}
\frac{d}{d t}\left[\begin{array}{cc}
M_{1} & M_{2} \\
0 & M_{4}
\end{array}\right]\left[\begin{array}{l}
x(t) \\
y(t)
\end{array}\right]+\left[\begin{array}{ll}
L_{1} & L_{2} \\
L_{3} & L_{4}
\end{array}\right]\left[\begin{array}{l}
x(t) \\
y(t)
\end{array}\right]=f(t)\left[\begin{array}{l}
z_{1} \\
z_{2}
\end{array}\right], \\
\forall t \in[0, \tau], \\
\left(M_{1} x+M_{2} y\right)(0)=M_{1} x_{0}+M_{2} y_{0}, \\
\left(M_{4} y\right)(0)=M_{4} y_{0}, \\
\Phi_{1}\left[M_{1} x(t)+M_{2} y(t)\right]+\Phi_{2}\left[M_{4} y(t)\right]=g(t), \\
\forall t \in[0, \tau],
\end{array}
$$

admits a unique global solution $(x, y, f) \in C^{\theta}\left([0, \tau] ; \mathscr{D}\left(L_{1}\right)\right) \times$ $C^{\theta}\left([0, \tau] ; \mathscr{D}\left(L_{4}\right)\right) \times C^{\theta}([0, \tau] ; \mathbb{R})$, such that $M_{1} x+M_{2} y \in$ $C^{1+\theta}([0, \tau] ; X), M_{4} y \in C^{1+\theta}([0, \tau] ; X)$.

Remark 19. When $M_{2}=0$, we can choose $\delta=1$; thus, the first two conditions in the statement of Theorem 18 involving $\alpha, \beta_{1}, \beta_{4}, \beta_{5}, \delta$ reduce to $\alpha+\beta_{1}+\beta_{5}>5 / 2$ and $\beta_{1}+\beta_{4}+\delta>2$. 
Remark 20. We could easily establish a result at all analogous to the one in Proposition 16, concerning this more general case.

We are now in a position to face the general identification problem:

$$
\begin{gathered}
\frac{d}{d t}\left[\begin{array}{ll}
M_{1} & M_{2} \\
M_{3} & M_{4}
\end{array}\right]\left[\begin{array}{l}
x(t) \\
y(t)
\end{array}\right] \\
+\left[\begin{array}{ll}
L_{1} & L_{2} \\
L_{3} & L_{4}
\end{array}\right]\left[\begin{array}{l}
x(t) \\
y(t)
\end{array}\right]=f(t)\left[\begin{array}{l}
z_{1} \\
z_{2}
\end{array}\right], \quad \forall t \in[0, \tau], \\
{\left[\begin{array}{ll}
M_{1} & M_{2} \\
M_{3} & M_{4}
\end{array}\right]\left[\begin{array}{l}
x(0) \\
y(0)
\end{array}\right]=\left[\begin{array}{ll}
M_{1} & M_{2} \\
M_{3} & M_{4}
\end{array}\right]\left[\begin{array}{l}
x_{0} \\
y_{0}
\end{array}\right], \quad(127)} \\
\Phi_{1}\left[M_{1} x(t)+M_{2} y(t)\right]+\Phi_{2}\left[M_{3} x(t)+M_{4} y(t)\right]=g(t), \\
\forall t \in[0, \tau] .
\end{gathered}
$$

To this end, we will assume that

$$
M_{1}, M_{3} \text { have bounded inverses, }
$$$$
\text { with } \mathscr{D}\left(M_{1}\right) \subseteq \mathscr{D}\left(M_{3}\right) \text {. }
$$

and the additional information

$$
\begin{array}{r}
\Psi_{1}\left[M_{1} x(t)+M_{2} y(t)\right] \\
+\Psi_{2}\left[\left(M_{2}-M_{1} M_{3}^{-1} M_{4}\right) y(t)\right]=g(t), \\
\forall t \in[0, \tau],
\end{array}
$$

Multiply the second equation in the system by $M_{1} M_{3}^{-1}$ and substract the obtained equation from the first one. We obtain the following system equivalent to (126):

$$
\begin{aligned}
& \frac{d}{d t}\left[\begin{array}{cc}
M_{1} & M_{2} \\
0 & M_{2}-M_{1} M_{3}^{-1} M_{4}
\end{array}\right]\left[\begin{array}{l}
x(t) \\
y(t)
\end{array}\right] \\
& \quad+\left[\begin{array}{c}
L_{1} \\
L_{2}-M_{1} M_{3}^{-1} L_{3}
\end{array}\right]\left[\begin{array}{l}
x(t) \\
L_{2}-M_{1} M_{3}^{-1} L_{4}
\end{array}\right] \\
& \quad=f(t)\left[\begin{array}{c}
z_{1} \\
z_{1}-M_{1} M_{3}^{-1} z_{2}
\end{array}\right], \quad \forall t \in[0, \tau],
\end{aligned}
$$

where $\Psi=\left(\Psi_{1}, \Psi_{2}\right)$ is related to $\Phi=\left(\Phi_{1}, \Phi_{2}\right)$ by

$$
\Psi=\Phi\left[\begin{array}{cc}
1 & 0 \\
M_{3} M_{1}^{-1} & -M_{3} M_{1}^{-1}
\end{array}\right] \text {, }
$$

so that

$$
\begin{aligned}
& \Psi\left[M_{1} x(t)+M_{2} y(t),\left(M_{2}-M_{1} M_{3}^{-1} M_{4}\right) y(t)\right] \\
& =\Psi_{1}\left[M_{1} x(t)+M_{2} y(t)\right]+\Psi_{2}\left[\left(M_{2}-M_{1} M_{3}^{-1} M_{4}\right) y(t)\right] \\
& =\Phi\left[\begin{array}{cc}
1 & 0 \\
M_{3} M_{1}^{-1} & -M_{3} M_{1}^{-1}
\end{array}\right]\left[\begin{array}{c}
M_{1} x(t)+M_{2} y(t) \\
\left(M_{2}-M_{1} M_{3}^{-1} M_{4}\right) y(t)
\end{array}\right] \\
& =\Phi\left[\begin{array}{c}
M_{1} x(t)+M_{2} y(t) \\
M_{3} M_{1}^{-1} M_{1} x(t)+M_{3} M_{1}^{-1} M_{2} y(t)-M_{3} M_{1}^{-1} M_{2} y(t)+M_{4} y(t)
\end{array}\right] \\
& =\Phi\left[\begin{array}{l}
M_{1} x(t)+M_{2} y(t) \\
M_{3} x(t)+M_{4} y(t)
\end{array}\right] \\
& =\Phi_{1}\left[M_{1} x(t)+M_{2} y(t)\right]+\Phi_{2}\left[M_{3} x(t)+M_{4} y(t)\right] .
\end{aligned}
$$


Notice that the operator $M_{2}-M_{1} M_{3}^{-1} M_{4}$ may have no bounded inverse. Our next step is to translate to this case the conditions established in Theorem 14. Assume, in addition to (95),

$$
\begin{aligned}
& \|\left(M_{2}-M_{1} M_{3}^{-1} M_{4}\right) \\
& \quad \times\left(z\left(M_{2}-M_{1} M_{3}^{-1} M_{4}\right)+L_{2}-M_{1} M_{3}^{-1} L_{4}\right)^{-1} \|_{\mathscr{L}(X)} \\
& \leq C(1+|z|)^{-\beta_{4}}, \quad \forall z \in \Sigma_{\alpha}, \\
& \left\|M_{2} x\right\| \leq C\left\|\left(M_{2}-M_{1} M_{3}^{-1} M_{4}\right) x\right\|^{\delta}\|x\|^{1-\delta}, \quad 0<\delta \leq 1, \\
& x \in D\left(M_{2}-M_{1} M_{3}^{-1} M_{4}\right),
\end{aligned}
$$

$$
L_{1}-M_{1} M_{3}^{-1} L_{3} \text { has a bounded extension to } X \text {, }
$$

and thus $\delta_{1}=1$. Compute now

$$
\begin{aligned}
& \Psi\left[\begin{array}{c}
z_{1} \\
z_{1}-M_{1} M_{3}^{-1} z_{2}
\end{array}\right] \\
& \quad=\Phi\left[\begin{array}{cc}
1 & 0 \\
M_{3} M_{1}^{-1} & -M_{3} M_{1}^{-1}
\end{array}\right]\left[\begin{array}{c}
z_{1} \\
z_{1}-M_{1} M_{3}^{-1} z_{2}
\end{array}\right] \\
& \quad=\Phi\left[\begin{array}{c}
z_{1} \\
M_{3} M_{1}^{-1} z_{1}-M_{3} M_{1}^{-1} z_{1}+z_{2}
\end{array}\right] \\
& \quad=\Phi_{1}\left[z_{1}\right]+\Phi_{2}\left[z_{2}\right]
\end{aligned}
$$

as desired.

Therefore, we are in a position to establish the following result.

Theorem 21. Suppose that operators $L_{i}, M_{i}$ satisfy (95), (135), (136), and (137) and that $M_{1}, M_{3} \in \mathscr{L}(X)$ have bounded inverses, $\beta_{1}+\beta_{4}+\delta>2$, and $\alpha+\beta_{1}+\beta_{4}+\delta>7 / 2$, $\theta \in\left(4-\alpha-\beta_{1}-\beta_{4}-\delta, \alpha+\beta_{1}+\beta_{4}+\delta-3\right)$,

$$
\begin{gathered}
{\left[\begin{array}{l}
z_{1} \\
z_{2}
\end{array}\right]=\left[\begin{array}{l}
M_{1} z_{1}^{*}+M_{2} z_{2}^{*} \\
M_{3} z_{1}^{*}+M_{4} z_{2}^{*}
\end{array}\right],} \\
{\left[\begin{array}{ll}
L_{1} & L_{2} \\
L_{3} & L_{4}
\end{array}\right]\left[\begin{array}{l}
x_{0} \\
y_{0}
\end{array}\right]=\left[\begin{array}{ll}
M_{1} & M_{2} \\
M_{3} & M_{4}
\end{array}\right]\left[\begin{array}{l}
x_{0}^{*} \\
y_{0}^{*}
\end{array}\right],}
\end{gathered}
$$

where $z_{1}^{*}, x_{0}^{*} \in \mathscr{D}\left(L_{1}\right), z_{2}^{*}, y_{0}^{*} \in \mathscr{D}\left(L_{4}\right), g \in C^{1+\theta}([0, \tau] ; \mathbb{R})$, and (111) holds together with the compatibility relation

$$
\Phi_{1}\left[M_{1} x_{0}+M_{2} y_{0}\right]+\Phi_{2}\left[M_{3} x_{0}+M_{4} y_{0}\right]=g(0) .
$$

Then the identification Problems (126)-(128) admit a unique strict global solution $(x, y, f) \in C^{\theta}\left([0, \tau] ; \mathscr{D}\left(L_{1}\right)\right) \times C^{\theta}([0, \tau]$; $\left.\mathscr{D}\left(L_{4}\right)\right) \times C^{\theta}([0, \tau] ; \mathbb{R})$, with $M_{1} x+M_{2} y \in C^{1+\theta}([0, \tau] ; X)$, $M_{3} x+M_{4} y \in C^{1+\theta}([0, \tau] ; X)$.

Proof. It is a simple rewriting of the result in Theorem 18 as applied to Problems (130)-(132), taking into account the relation to the starting Problems (126)-(128).
Of course, the preceding results apply to the abstract strongly degenerate elliptic-parabolic system

$$
\begin{gathered}
\frac{d}{d t}\left[\begin{array}{cc}
M_{1} & M_{2} \\
0 & 0
\end{array}\right]\left[\begin{array}{l}
x(t) \\
y(t)
\end{array}\right]+\left[\begin{array}{ll}
L_{1} & L_{2} \\
L_{3} & L_{4}
\end{array}\right]\left[\begin{array}{l}
x(t) \\
y(t)
\end{array}\right] \\
=f(t)\left[\begin{array}{l}
z_{1} \\
z_{2}
\end{array}\right], \quad \forall t \in[0, \tau], \\
\left(M_{1} x+M_{2} y\right)(0)=M_{1} x_{0}+M_{2} y_{0}, \\
\Phi\left[M_{1} x(t)+M_{2} y(t)\right]=g(t), \quad \forall t \in[0, \tau] .
\end{gathered}
$$

However, in view of this generality, the corresponding assumptions would yield the restrictions $z_{2}=0$ and $M_{2}=0$. To overcome this difficulty we will make suitable assumptions on the operators involved.

Clearly, if $z_{2}=0$ and $L_{4}$ has a bounded inverse, the second equation in (141) gives $y(t)=-L_{4}^{-1} L_{3} x(t)$, so that (141)-(143) reduces to the identification problem:

$$
\begin{aligned}
& \frac{d}{d t}\left(M_{1}-M_{2} L_{4}^{-1} L_{3}\right) x(t)+\left(L_{1}-L_{2} L_{4}^{-1} L_{3}\right) x(t) \\
& =f(t) z_{1}, \quad 0 \leq t \leq \tau, \\
& \quad\left(M_{1}-M_{2} L_{4}^{-1} L_{3}\right) x(0)=M_{1} x_{0}+M_{2} y_{0}, \\
& \Phi\left[\left(M_{1}-M_{2} L_{4}^{-1} L_{3}\right) x(t)\right]=g(t), \quad 0 \leq t \leq \tau .
\end{aligned}
$$

Observe that the compatibility relation $y_{0}=L_{4}^{-1} L_{3} x_{0}$ must hold. On the other hand, all the results in Propositions 29 and 32 apply provided that operators $A=L_{1}-L_{2} L_{4}^{-1} L_{3}$ and $B=$ $M_{1}-M_{2} L_{4}^{-1} L_{3}$ satisfy the assumptions described there.

If $z_{2} \neq 0$, so that $y_{4}(t)=f(t) L_{4}^{-1} z_{2}-L_{4}^{-1} L_{3} x(t)$, we arrive at the following identification problem:

$$
\begin{gathered}
\frac{d}{d t}\left(M_{1} x(t)-M_{2} L_{4}^{-1} L_{3} x(t)+f(t) M_{2} L_{4}^{-1} z_{2}\right) \\
\quad+\left(L_{1}-L_{2} L_{4}^{-1} L_{3}\right) x(t) \\
=f(t)\left[z_{1}-L_{2} L_{4}^{-1} z_{2}\right], \quad 0 \leq t \leq \tau, \\
{\left[\left(M_{1}-M_{2} L_{4}^{-1} L_{3}\right) x+f(t) M_{2} L_{4}^{-1} z_{2}\right](0)=M_{1} x_{0}+M_{2} y_{0},} \\
\Phi\left[\left(M_{1}-M_{2} L_{4}^{-1} L_{3}\right) x(t)+f(t) M_{2} L_{4}^{-1} z_{2}\right] \\
=g(t), \quad 0 \leq t \leq \tau .
\end{gathered}
$$

Notice the extra difficulty arising from the fact that the unknown term $f(t)$ is not supposed to be differentiable. However, if $M_{1}-M_{2} L_{4}^{-1} L_{3}$ has a bounded inverse, we can introduce the new unknown:

$$
\left(M_{1}-M_{2} L_{4}^{-1} L_{3}\right) x(t)+f(t) M_{2} L_{4}^{-1} z_{2}=\xi(t),
$$

so that

$$
\begin{aligned}
x(t)= & \left(M_{1}-M_{2} L_{4}^{-1} L_{3}\right)^{-1} \xi(t) \\
& -f(t)\left(M_{1}-M_{2} L_{4}^{-1} L_{3}\right)^{-1} M_{2} L_{4}^{-1} z_{2} .
\end{aligned}
$$


Then (145) reads equivalently

$$
\begin{gathered}
\frac{d}{d t} \xi(t)+\left(L_{1}-L_{2} L_{4}^{-1} L_{3}\right)\left(M_{1}-M_{2} L_{4}^{-1} L_{3}\right)^{-1} \xi(t) \\
=f(t) \bar{z}, \quad 0 \leq t \leq \tau, \\
\xi(0)=M_{1} x_{0}+M_{2} y_{0}, \\
\Phi[\xi(t)]=g(t), \quad 0 \leq t \leq \tau,
\end{gathered}
$$

where

$$
\begin{aligned}
\bar{z}=[ & z_{1}-L_{2} L_{4}^{-1} z_{2} \\
& \left.+\left(L_{1}-L_{2} L_{4}^{-1} L_{3}\right)\left(M_{1}-M_{2} L_{4}^{-1} L_{3}\right)^{-1} M_{2} L_{4}^{-1} z_{2}\right]
\end{aligned}
$$

This is a regular identification problem if, for example, $\left(L_{1}-\right.$ $\left.L_{2} L_{4}^{-1} L_{3}\right)\left(M_{1}-M_{2} L_{4}^{-1} L_{3}\right)^{-1}$ is a bounded operator. Indeed, we have the following.

Corollary 22. Let $M_{1}-M_{2} L_{4}^{-1} L_{3}$ have a bounded inverse and let $\left(L_{1}-L_{2} L_{4}^{-1} L_{3}\right)\left(M_{1}-M_{2} L_{4}^{-1} L_{3}\right)^{-1}$ be bounded. Let the compatibility relation $\Phi\left[M_{1} x_{0}+M_{2} y_{0}\right]=g(0)$ hold together with $\Phi[\bar{z}] \neq 0$. Then Problems (141)-(143) admit a unique strict solution $(x, y, f) \in C^{\theta}\left([0, \tau] ; \mathscr{D}\left(L_{1}\right)\right) \times C^{\theta}\left([0, \tau] ; \mathscr{D}\left(L_{4}\right)\right) \times$ $C^{\theta}([0, \tau] ; \mathbb{R}), 0<\theta<1$.

Corollary 22 can, in fact, be refined weakening the assumption on $\left(L_{1}-L_{2} L_{4}^{-1} L_{3}\right)\left(M_{1}-M_{2} L_{4}^{-1} L_{3}\right)^{-1}$. For this purpose consider the system

$$
\begin{gathered}
M_{1} x+M_{2} y=\xi, \\
L_{3} x+L_{4} y=f(t) z_{2} .
\end{gathered}
$$

If operator $M_{1}-M_{2} L_{4}^{-1} L_{3}:=S$ has an inverse, then

$$
\begin{gathered}
x=S^{-1} \xi-S^{-1} M_{2} L_{4}^{-1} z_{2} f(t), \\
y=-L_{4}^{-1} L_{3} S^{-1} \xi+f(t)\left[L_{4}^{-1}+L_{4}^{-1} L_{3} S^{-1} M_{2} L_{4}^{-1}\right] z_{2} .
\end{gathered}
$$

Therefore, the pair $(\xi, f)$ satisfies the problem

$$
\begin{gathered}
\xi^{\prime}(t)+\left(L_{1}-L_{2} L_{4}^{-1} L_{3}\right) S^{-1} \xi(t)=f(t) \bar{z}, \quad 0 \leq t \leq \tau, \\
\xi(0)=M_{1} x_{0}+M_{2} y_{0}, \\
\Phi[\xi(t)]=g(t), \quad 0 \leq t \leq \tau,
\end{gathered}
$$

where

$$
\begin{gathered}
\Phi[\bar{z}] \neq 0, \\
\bar{z}:=z_{1}-L_{2} L_{4}^{-1} z_{2}+\left(L_{1}-L_{2} L_{4}^{-1} L_{3}\right) S^{-1} M_{2} L_{4}^{-1} z_{2} .
\end{gathered}
$$

That is, $(\xi, f)$ satisfies (148), as desired.

Let $\mathbb{A}=\left(L_{1}-L_{2} L_{4}^{-1} L_{3}\right) S^{-1}$. Then, from Theorem $2.1 \mathrm{in}$ [1] we deduce the following.
Theorem 23. Let $-\mathbb{A}$ be the generator of an analytic semigroup of negative type in $X, 0<\theta<\theta_{0}<1, \xi_{0}=M_{1} x_{0}+$ $M_{2} y_{0} \in \mathscr{D}_{\mathbb{A}}(\theta+1,+\infty), \bar{z} \in \mathscr{D}_{\mathbb{A}}\left(\theta_{0},+\infty\right), \Phi[\bar{z}] \neq 0, g \in$ $C^{1+\theta}([0, \tau] ; \mathbb{R}), \Phi\left[\xi_{0}\right]=g(0)$. Then Problems (141)-(143) admit a unique strict global solution $(x, y, f) \in C^{\theta}([0, \tau]$; $\left.\mathscr{D}\left(L_{1}\right)\right) \times C^{\theta}\left([0, \tau] ; \mathscr{D}\left(L_{4}\right)\right) \times C^{\theta}([0, \tau] ; \mathbb{R})$, such that $M_{1} x+$ $M_{2} y \in C^{1+\theta}([0, \tau] ; X)$.

Applying Corollary 31, we also obtain the following.

\section{Corollary 24. If}

$$
\left\|(z+\mathbb{A})^{-1}\right\|_{\mathscr{L}(X)} \leq C(1+|z|)^{-\beta}, \quad \forall z \in \Sigma_{1},
$$

where $\beta \in(1 / 2,1), \bar{z} \in \mathscr{D}(\mathbb{A}), \Phi[\bar{z}] \neq 0, \xi_{0}=M_{1} x_{0}+$ $M_{2} y_{0} \in \mathscr{D}\left(\mathbb{A}^{2}\right), \Phi\left[\xi_{0}\right]=g(0), g \in C^{1+\theta}([0, \tau] ; \mathbb{R}), 1-\beta<$ $\theta<\beta$, then Problems (141)-(143) admit a unique strict global solution $(x, y, f) \in C^{\theta}\left([0, \tau] ; \mathscr{D}\left(L_{1}\right)\right) \times C^{\theta}\left([0, \tau] ; \mathscr{D}\left(L_{4}\right)\right) \times$ $C^{\theta}([0, \tau] ; \mathbb{R})$, with $M_{1} x+M_{2} y \in C^{1+\theta}([0, \tau] ; X)$.

Example 25. Consider the identification problem:

$$
\begin{gathered}
\frac{d}{d t}\left[\begin{array}{ll}
I & I \\
0 & 0
\end{array}\right]\left[\begin{array}{l}
x(t) \\
y(t)
\end{array}\right]+\left[\begin{array}{ll}
\alpha L & \beta L \\
\gamma L & \delta L
\end{array}\right]\left[\begin{array}{l}
x(t) \\
y(t)
\end{array}\right] \\
=f(t)\left[\begin{array}{l}
z_{1} \\
z_{2}
\end{array}\right], \quad \forall t \in[0, \tau], \\
(x+y)(0)=\xi_{0}, \\
\Phi[(x+y)(t)]=g(t), \quad \forall t \in[0, \tau], \Phi\left[\xi_{0}\right]=g(0),
\end{gathered}
$$

where $L$ is a closed linear operator in the Banach space $X$ and $\alpha, \beta, \gamma, \delta \in \mathbb{C}$.

To apply Theorem 23 we set $S=((\delta-\gamma) / \delta) I$ and we note that $L_{1}-L_{2} L_{4}^{-1} L_{3}=((\alpha \delta-\beta \gamma) / \delta) L$, so that $\left(L_{1}-\right.$ $\left.L_{2} L_{4}^{-1} L_{3}\right) S^{-1}=((\alpha \delta-\beta \gamma) /(\delta-\gamma)) L$. Moreover,

$$
\bar{z}=\frac{(\delta-\gamma) z_{1}+(\alpha-\beta) z_{2}}{\delta-\gamma} .
$$

It follows that if $((\alpha \delta-\beta \gamma) /(-\delta+\gamma)) L$ generates an analytic semigroup of negative type and $(\delta-\gamma) z_{1}+(\alpha-\beta) z_{2} \epsilon$ $D_{L}\left(\theta_{0},+\infty\right), \Phi\left[(\delta-\gamma) z_{1}+(\alpha-\beta) z_{2}\right] \neq 0, g \in C^{1+\theta}([0, \tau] ; \mathbb{R})$, $0<\theta<\theta_{0}<1, \xi_{0} \in D_{L}(\theta+1, \infty)$, then the identification Problem (155) admits a unique global strict solution $(x, y, f) \in C^{\theta}([0, \tau] ; \mathscr{D}(L)) \times C^{\theta}([0, \tau] ; \mathscr{D}(L)) \times C^{\theta}([0, \tau] ; \mathbb{R})$, $x+y \in C^{1+\theta}([0, \tau] ; X)$. 
We stress that we have been able to determine a triplet of functions $u_{1}, u_{2}:[0, \tau] \times \Omega \rightarrow \mathbb{R}$ and $f:[0, \tau] \rightarrow \mathbb{R}$ in the following parabolic-elliptic identification problem:

$$
\begin{gathered}
D_{t}\left[u_{1}(x, t)+u_{2}(x, t)\right] \\
+\alpha L\left(x, D_{x}\right) u_{1}(x, t)+\beta L\left(x, D_{x}\right) u_{2}(x, t) \\
=f(t) z_{1}(x), \quad t \in[0, \tau], x \in \Omega, \\
\gamma L\left(x, D_{x}\right) u_{1}(x, t)+\delta L\left(x, D_{x}\right) u_{2}(x, t) \\
=f(t) z_{2}(x), \quad t \in[0, \tau], x \in \Omega, \\
u_{1}(x, t)+u_{2}(x, t) \\
=u_{1,0}(x)+u_{2,0}(x), \quad t \in[0, \tau], x \in \partial \Omega, \\
u_{1}(x, t)=u_{2}(x, t)=0, \quad x \in \partial \Omega, \\
\int_{\Omega} \psi(x)\left[u_{1}(x, t)+u_{2}(x, t)\right] d t=g(t), \quad t \in[0, \tau] .
\end{gathered}
$$

Here operator $L\left(x, D_{x}\right)$ is defined by

$$
\begin{aligned}
\mathscr{D}(L)=W^{2, p}(\Omega) \cap W_{0}^{1, p}(\Omega), \quad p \in(1,+\infty), \\
L u(x)=-\sum_{i, j=1}^{n} D_{x_{i}}\left[a_{i, j}(x) D_{x_{j}}\right] \\
+\sum_{i=1}^{n} a_{i}(x) D_{x_{i}}+a_{0}(x), \quad x \in \Omega,
\end{aligned}
$$

where $(\alpha \delta-\beta \gamma)(\gamma-\delta)^{-1}<0$ and the coefficients $a_{i, j}, a_{j}, a_{0}$ satisfy the following assumptions:

$$
\begin{gathered}
a_{i, j}, D_{x_{i}} a_{i, j}, a_{i}, D_{x_{i}} a_{i}, a_{0} \in C(\bar{\Omega}), \quad a_{i, j}(x)=a_{j, i}(x), \\
x \in \Omega, i, j=1, \ldots, n, \\
\sum_{i, j=1}^{n} a_{i, j}(x) \xi_{i} \xi_{j} \geq c_{0}|\xi|^{2}, \quad x \in \bar{\Omega}, \xi \in \mathbb{R}^{n}, \\
a_{0}(x)-\frac{1}{p} \sum_{i=1}^{n} D_{x_{i}} a_{i}(x) \geq c_{1}, \quad x \in \bar{\Omega} .
\end{gathered}
$$

The same argument applies when $L(x, D)$ is the opposite of the realization in $X=L_{\mu}^{p}\left(\mathbb{R}^{n}\right), p \in(1,+\infty)$, of the OrnsteinUhlenbeck operator $\mathscr{L}_{p}$, precisely

$$
\begin{array}{r}
\mathscr{D}(L)=W_{\mu}^{2, p}\left(\mathbb{R}^{n}\right), \quad p \in(1,+\infty), L=-\mathscr{L}_{p}+k I, \\
k \in \mathbb{R}_{+}
\end{array}
$$

(for the properties in $L_{\mu}^{p}, p \in(1,+\infty)$, cf. Problem 9).

In this case (161) changes to

$$
\int_{\Omega} \psi(x)\left[u_{1}(x, t)+u_{2}(x, t)\right] \mu(d x)=g(t), \quad t \in[0, \tau] .
$$

Example 26. Consider the identification problem:

$$
\begin{array}{r}
\frac{d}{d t}\left[\begin{array}{ll}
I & I \\
I & I
\end{array}\right]\left[\begin{array}{l}
x(t) \\
y(t)
\end{array}\right]+\left[\begin{array}{ll}
\alpha L & \beta L \\
\gamma L & \delta L
\end{array}\right]\left[\begin{array}{l}
x(t) \\
y(t)
\end{array}\right] \\
=f(t)\left[\begin{array}{l}
z_{1} \\
z_{2}
\end{array}\right], \quad \forall t \in[0, \tau], \\
(x+y)(0)=\xi_{0}, \\
\Phi_{1}[(x+y)(t)]+\Phi_{2}[(x+y)(t)] \\
=\left(\Phi_{1}+\Phi_{2}\right)[(x+y)(t)]=g(t), \\
\forall t \in[0, \tau],
\end{array}
$$

with $\left(\Phi_{1}+\Phi_{2}\right)\left(\xi_{0}\right)=g(0)$.

Such a problem is easily reduced to an equivalent problem related to the integral differential equation:

$$
\begin{aligned}
\frac{d}{d t} & {\left[\begin{array}{ll}
I & I \\
0 & 0
\end{array}\right]\left[\begin{array}{l}
x(t) \\
y(t)
\end{array}\right]+\left[\begin{array}{cc}
\alpha L & \beta L \\
(\alpha-\gamma) L & (\beta-\delta) L
\end{array}\right]\left[\begin{array}{l}
x(t) \\
y(t)
\end{array}\right] } \\
& =f(t)\left[\begin{array}{c}
z_{1} \\
z_{1}-z_{2}
\end{array}\right], \quad \forall t \in[0, \tau] .
\end{aligned}
$$

Consequently, we have come back to Example 25 with $\gamma, \delta, z_{2}$ being replaced by $\alpha-\gamma, \beta-\delta, z_{1}-z_{2}$, respectively.

Taking this into account, the same conclusions as Example 25 can be obtained, provided that $\alpha \delta-\beta \gamma \neq 0$, $\alpha-\beta-\gamma+\delta \neq 0,-(\alpha \delta-\beta \gamma)(\alpha-\beta-\gamma+\delta)^{-1} L$ generates a holomorphic semigroup in $X,(\gamma-\delta) z_{1}-(\alpha-\beta) z_{2} \epsilon$ $D_{L}\left(\theta_{0},+\infty\right),\left(\Phi_{1}+\Phi_{2}\right)\left[(\gamma-\delta) z_{1}-(\alpha-\beta) z_{2}\right] \neq 0, \xi_{0} \epsilon$ $D_{L}(\theta+1,+\infty)$. Then the identification problem above admits a unique global strict solution $(x, y, f) \in C^{\theta}([0, \tau] ; \mathscr{D}(L)) \times$ $C^{\theta}([0, \tau] ; \mathscr{D}(L)) \times C^{\theta}([0, \tau] ; \mathbb{R}), x+y \in C^{1+\theta}([0, \tau] ; X)$.

Consider now the identification problem:

$$
\begin{array}{r}
\frac{d}{d t}\left[\begin{array}{cc}
0 & 0 \\
M_{3} & 0
\end{array}\right]\left[\begin{array}{l}
x(t) \\
y(t)
\end{array}\right]+\left[\begin{array}{ll}
L_{1} & L_{2} \\
L_{3} & L_{4}
\end{array}\right]\left[\begin{array}{l}
x(t) \\
y(t)
\end{array}\right]=f(t)\left[\begin{array}{l}
z_{1} \\
z_{2}
\end{array}\right], \\
\forall t \in[0, \tau], \\
\left(M_{3} x\right)(0)=M_{3} x_{0}, \\
\Phi\left[M_{3} x(t)\right]=g(t), \quad \forall t \in[0, \tau], \Phi\left[M_{3} x_{0}\right]=g(0) .
\end{array}
$$

Under the assumption that $L_{2}$ have a bounded inverse we get $y=f(t) L_{2}^{-1} z_{1}-L_{2}^{-1} L_{1} x$. Therefore we obtain the following differential equation for the single unknown $x$ :

$$
\frac{d}{d t}\left(M_{3} x\right)(t)+\left(L_{3}-L_{4} L_{2}^{-1} L_{1}\right) x=f(t)\left[z_{2}-L_{4} L_{2}^{-1} z_{1}\right] .
$$

Now it suffices to suppose that the pair $\left(M_{3}, L_{3}-L_{4} L_{2}^{-1} L_{1}\right)$ satisfies the properties described in [1] or in Section 5 in order to obtain existence and uniqueness for the given identification problem. 
If $M_{3}$ has an inverse $M_{3}^{-1}$, introduce function $\xi$ defined by $M_{3} x=\xi$, so that

$$
\xi^{\prime}(t)+L_{3} M_{3}^{-1} \xi(t)+L_{4} y(t)=f(t) z_{2} .
$$

Assuming that $L_{4}$ has a bounded inverse, we have

$$
y(t)=f(t) L_{4}^{-1} z_{2}-L_{4}^{-1} \xi^{\prime}(t)-L_{4}^{-1} L_{3} M_{3}^{-1} \xi(t) .
$$

Substituting in the first equation of the system, we get

$$
\begin{aligned}
& L_{1} M_{3}^{-1} \xi(t) \\
& \quad=L_{2} L_{4}^{-1} \xi^{\prime}(t)-L_{2} L_{4}^{-1} L_{3} M_{3}^{-1} \xi(t) \\
& \quad=f(t)\left[z_{1}-L_{2} L_{4}^{-1} z_{2}\right] .
\end{aligned}
$$

That is, we have obtained the following differential identification problem for $\xi$ :

$$
\begin{gathered}
L_{2} L_{4}^{-1} \xi^{\prime}(t)+\left[L_{2} L_{4}^{-1} L_{3}-L_{1}\right] M_{3}^{-1} \xi(t) \\
=f(t)\left[L_{2} L_{4}^{-1} z_{2}-z_{1}\right], \quad 0 \leq t \leq \tau, \\
\xi(0)=M_{3} x_{0}, \\
\Phi[\xi(t)]=g(t), \quad 0 \leq t \leq \tau .
\end{gathered}
$$

This identification problem might be treated similarly under the obvious hypothesis $L_{2} L_{4}^{-1}$ to have a bounded inverse. But if $L_{2}$ is invertible we come back to the first case.

The assumption requiring $L_{2}$ to be invertible seems really essential in some sense. As an example, take

$$
\begin{gathered}
\frac{d}{d t}\left[\begin{array}{cc}
0 & 0 \\
M_{3} & 0
\end{array}\right]\left[\begin{array}{l}
x(t) \\
y(t)
\end{array}\right]+\left[\begin{array}{cc}
L_{1} & 0 \\
0 & L_{4}
\end{array}\right]\left[\begin{array}{l}
x(t) \\
y(t)
\end{array}\right]=f(t)\left[\begin{array}{l}
z_{1} \\
z_{2}
\end{array}\right], \\
\forall t \in[0, \tau], \\
\left(M_{3} x\right)(0)=\xi_{0}, \\
\Phi\left[M_{3} x(t)\right]=g(t), \quad \forall t \in[0, \tau], \Phi\left[\xi_{0}\right]=g(0),
\end{gathered}
$$

where $L_{1}$ and $L_{2}$ admit bounded inverses. Then $x(t)=$ $f(t) L_{1}^{-1} z_{1}$ implies $\Phi\left[M_{3} x(t)\right]=\Phi\left[f(t) M_{3} L_{1}^{-1} z_{1}\right]=g(t)$. Hence, if $\Phi\left[M_{3} L_{1}^{-1} z_{1}\right] \neq 0$, then we necessarily have

$$
f(t)=\frac{g(t)}{\Phi\left[M_{3} L_{1}^{-1} z_{1}\right]}, \quad x(t)=\frac{g(t)}{\Phi\left[M_{3} L_{1}^{-1} z_{1}\right]} L_{1}^{-1} z_{1} .
$$

Moreover, the second equation furnishes uniquely $y(t)$. This shows the importance of the invertibility of $M_{3}$, as expected. Notice too that no assumption like semigroup generation is required to operators $L_{1}$ and $L_{2}$.

Example 27. Let $A, B$ be two bounded linear operators in $X$. Observe first that the identification problem

$$
\begin{gathered}
\frac{d}{d t}\left[\begin{array}{ll}
A & B \\
A & B
\end{array}\right]\left[\begin{array}{l}
x(t) \\
y(t)
\end{array}\right]+\left[\begin{array}{ll}
L_{1} & L_{2} \\
L_{3} & L_{4}
\end{array}\right]\left[\begin{array}{l}
x(t) \\
y(t)
\end{array}\right] \\
=f(t)\left[\begin{array}{l}
z_{1} \\
z_{2}
\end{array}\right], \quad \forall t \in[0, \tau], \\
(A x+B y)(0)=\xi_{0}, \\
\Phi[A x(t)+B y(t)]=g(t), \quad \forall t \in[0, \tau],
\end{gathered}
$$

is equivalent to the problem

$$
\begin{gathered}
\frac{d}{d t}\left[\begin{array}{cc}
A & B \\
0 & 0
\end{array}\right]\left[\begin{array}{l}
x(t) \\
y(t)
\end{array}\right]+\left[\begin{array}{cc}
L_{1} & L_{2} \\
L_{1}-L_{3} & L_{2}-L_{4}
\end{array}\right]\left[\begin{array}{l}
x(t) \\
y(t)
\end{array}\right] \\
=f(t)\left[\begin{array}{c}
z_{1} \\
z_{1}-z_{2}
\end{array}\right], \quad \forall t \in[0, \tau] \\
(A x+B y)(0)=\xi_{0}, \\
\Phi[A x(t)+B y(t)]=g(t), \quad \forall t \in[0, \tau] .
\end{gathered}
$$

Thus we can apply both Theorem 23 and Corollary 22. Operator $\mathbb{A}$ is now given by $\left(L_{1}-L_{2}\left(L_{2}-L_{4}\right)^{-1}\left(L_{1}-L_{3}\right)\right)(A-$ $\left.B\left(L_{2}-L_{4}\right)^{-1}\left(L_{1}-L_{3}\right)\right)^{-1}$, while $\bar{z}$ is given by $\bar{z}=z_{1}-L_{2}\left(L_{2}-\right.$ $\left.L_{4}\right)^{-1}\left(z_{1}-z_{2}\right)+\mathbb{A} B\left(L_{2}-L_{4}\right)^{-1}\left(z_{1}-z_{2}\right)$.

Example 28. Let $A, B$ be two bounded linear operators in $X$, with $0 \in \rho(B), 0 \in \rho(A+k B)$ for some $k \neq 0$.

Consider the identification problem:

$$
\begin{gathered}
\frac{d}{d t}\left[\begin{array}{ll}
A & A \\
B & B
\end{array}\right]\left[\begin{array}{l}
x(t) \\
y(t)
\end{array}\right]+\left[\begin{array}{ll}
L_{1} & L_{2} \\
L_{3} & L_{4}
\end{array}\right]\left[\begin{array}{l}
x(t) \\
y(t)
\end{array}\right] \\
=f(t)\left[\begin{array}{l}
z_{1} \\
z_{1}
\end{array}\right], \quad \forall t \in[0, \tau], \\
A(x+y)(0)=A \xi_{0}, \quad B(x+y)(0)=B \eta_{0}, \\
\Phi_{1}[A(x+y)(t)]+\Phi_{2}[B(x+y)(t)] \\
=g(t), \quad \forall t \in[0, \tau],
\end{gathered}
$$

with a compatibility relation $g(0)=\Phi_{1}\left[A \xi_{0}\right]+\Phi_{2}\left[B \eta_{0}\right]$, $A \xi_{0}=A \eta_{0}$.

Note that, under our assumptions on $A$ and $B$, such a problem is equivalent to the following:

$$
\begin{aligned}
& \frac{d}{d t}\left[\begin{array}{ll}
I & I \\
I & I
\end{array}\right]\left[\begin{array}{l}
x(t) \\
y(t)
\end{array}\right] \\
& \quad+\left[\begin{array}{cc}
(A+k B)^{-1}\left(L_{1}+k L_{3}\right) & \left.(A+k B)^{-1}\left(L_{2}+k L_{4}\right)\right] \\
B^{-1} L_{3} & B^{-1} L_{4}
\end{array}\right] \\
& \quad=f(t)\left[\begin{array}{c}
(A+k B)^{-1}\left(z_{1}+k z_{2}\right) \\
B^{-1} z_{2}
\end{array}\right], \quad \forall t \in[0, \tau], \\
& \quad(x+y)(0)=(A+k B)^{-1}\left[A \xi_{0}+k B \eta_{0}\right]=\eta_{0}, \\
& \Psi_{1}[x(t)+y(t)]+\Psi_{2}[x(t)+y(t)]=g(t), \quad \forall t \in[0, \tau],
\end{aligned}
$$

where $\Psi_{1}=\Phi_{1} A, \Psi_{2}=\Phi_{2} B$. 
Since, in turn, this identification problem is equivalent to

$$
\begin{aligned}
& \frac{d}{d t}\left[\begin{array}{ll}
I & I \\
0 & 0
\end{array}\right]\left[\begin{array}{l}
x(t) \\
y(t)
\end{array}\right] \\
& +\left[\begin{array}{cc}
(A+k B)^{-1}\left(L_{1}+k L_{3}\right) & (A+k B)^{-1}\left(L_{2}+k L_{4}\right) \\
(A+k B)^{-1}\left(L_{1}+k L_{3}\right)-B^{-1} L_{3} & (A+k B)^{-1}\left(L_{2}+k L_{4}\right)-B^{-1} L_{4}
\end{array}\right]\left[\begin{array}{l}
x(t) \\
y(t)
\end{array}\right] \\
& =f(t)\left[\begin{array}{c}
(A+k B)^{-1}\left(z_{1}+k z_{2}\right) \\
(A+k B)^{-1}\left(z_{1}+k z_{2}\right)-B^{-1} z_{2}
\end{array}\right], \quad \forall t \in[0, \tau] \\
& (x+y)(0)=(A+k B)^{-1}\left[A \xi_{0}+k B \eta_{0}\right]=\eta_{0}, \\
& \left(\Psi_{1}+\Psi_{2}\right)[(x+y)(t)]=g(t), \quad \forall t \in[0, \tau] .
\end{aligned}
$$

Theorem 23 and Corollary 24 run as well.

As an example, let $X=L^{p}(\Omega), 1<p<+\infty, \Omega$ being a domain in $\mathbb{R}^{n}$ of class $C^{2}$. Let $L=-\Delta$ be the laplacian in $X$ endowed with Dirichlet boundary conditions, $L_{1}=\alpha L$, and let $L_{2}=\beta L, L_{3}=\gamma L, L_{4}=\delta L, \alpha, \beta, \gamma, \delta \in \mathbb{C}$. Let $m$ and $n$ be two real-valued continuous functions on $\bar{\Omega}, n(x) \geq c>0, m$ being possibly negative. Let $k \in \mathbb{R}$ be such that $m(x)+k n(x)>$ 0 for all $x \in \bar{\Omega}$. Given $z_{1}, z_{2} \in L^{p}(\Omega)$, our identification problem consists in finding a triplet $(u, v, f)$ such that

$$
\begin{aligned}
& D_{t}(m(x)(u(x, t)+v(x, t)))-\alpha \Delta u(x, t)-\beta \Delta v(x, t) \\
& \quad=f(t) z_{1}(x), \quad t \in[0, \tau], \quad x \in \Omega, \\
& D_{t}(n(x)(u(x, t)+v(x, t)))-\gamma \Delta u(x, t)-\delta \Delta v(x, t) \\
& \quad=f(t) z_{2}(x), \quad t \in[0, \tau], \quad x \in \Omega, \\
& \quad u(x, t)=v(x, t)=0, \quad t \in[0, \tau], \quad x \in \partial \Omega, \\
& m(x)[u(x, 0)+v(x, 0)]=m(x) \xi_{0}(x), \quad x \in \Omega, \\
& \quad n(x)[u(x, 0)+v(x, 0)]=n(x) \eta_{0}(x), \quad x \in \Omega, \\
& \int_{\Omega}\left[\eta_{1}(x) m(x)+\eta_{2}(x) n(x)\right][u(x, t)+v(x, t)] d t \\
& \quad=g(t), \quad t \in[0, \tau],
\end{aligned}
$$

$\eta_{1}, \eta_{2}$ being given functions in $L^{q}(\Omega), 1 / p+1 / q=1$, with $\int_{\Omega}\left[\eta_{1}(x) m(x)+\eta_{2}(x) n(x)\right] \xi_{0}(x) d x=g(0), m(x)\left[\xi_{0}(x)-\right.$ $\left.\eta_{0}(x)\right] \equiv 0$. If $\nu(x):=m(x)+k n(x)$, let

$$
\begin{array}{cc}
a(x)=\frac{\alpha+k \gamma}{\gamma(x)}, & b(x)=\frac{\beta+k \delta}{\nu(x)}, \\
c(x)=\frac{\alpha+k \gamma}{\nu(x)}-\frac{\gamma}{n(x)}, & d(x)=\frac{\beta+k \delta}{\nu(x)}-\frac{\gamma}{n(x)} .
\end{array}
$$

Then $S^{-1}=L^{-1}(d /(d-c)) L, L_{1}-L_{2} L_{4}^{-1} L_{3}=((a d-b c) / d) L$, so that $-\mathbb{A}=-((a d-b c) /(d-c)) L=((a d-b c) /(d-c)) \Delta$ is assumed to generate an analytic semigroup in $L^{p}(\Omega)$.
Since

$$
\begin{aligned}
\bar{z}(x)= & \frac{z_{1}(x)+k z_{2}(x)}{\nu(x)}-\frac{b(x)}{d(x)}\left[\frac{z_{1}(x)+k z_{2}(x)}{v(x)}-\frac{z_{2}(x)}{n(x)}\right] \\
& +\frac{a d-b c}{d-c}\left(\frac{\beta+k \delta}{r(x)}-\frac{\delta}{n(x)}\right)^{-1} \\
& \times\left[\frac{z_{1}(x)+k z_{2}(x)}{v(x)}-\frac{z_{2}(x)}{n(x)}\right],
\end{aligned}
$$

our previous abstract assumptions read as

$$
\begin{gathered}
\bar{z} \in \mathscr{D}_{\mathbb{A}}\left(\theta_{0}, \infty\right)=D_{\Delta}\left(\theta_{0}, \infty\right) \\
\int_{\Omega}\left[\eta_{1}(x) m(x)+\eta_{2}(x) n(x)\right] \eta_{0}(x) d x=g(0) \\
\int_{\Omega}\left[\eta_{1}(x) m(x)+\eta_{2}(x) n(x)\right] \bar{z}(x) d x \neq 0 .
\end{gathered}
$$

Suppose $\alpha, \beta, \gamma, \delta \in \mathbb{R}$. Trivial computations show that

$$
\begin{aligned}
& \frac{a d-b c}{d-c} \\
& =((\alpha+k \gamma)(\beta+k \delta) n(x)-(\alpha+k \gamma) \delta \nu(x) \\
& \left.\quad-(\alpha+k \gamma) n(x) \nu(x)+\gamma \nu^{2}(x)\right) \\
& \quad \times(\nu(x) n(x)[\beta-\alpha+k(\delta-\gamma)]+\nu(x)(\gamma-\delta))^{-1} \\
& =\omega(x) .
\end{aligned}
$$

We need $\omega(x) \geq c>0$ for all $x \in \bar{\Omega}$. Therefore, all the required elements are determined.

Clearly, if - $\mathbb{A}$ generates an infinitely differentiable semigroup

$$
\left\|(z+\mathbb{A})^{-1}\right\|_{\mathscr{L}(X)} \leq C(1+|z|)^{-\beta}, \quad \forall z \in \Sigma_{1}, \quad \beta \in\left(\frac{1}{2}, 1\right),
$$

we are compelled to require $\bar{z} \in \mathscr{D}(\mathbb{A})$, as in Corollary 24, (cf. $[10,13,14])$. 


\section{Some Improvements of Known Results}

For the reader's convenience, we report here the main results in [1] with some minor improvements.

Proposition 29. Let $0<\beta \leq \alpha \leq 1, \alpha+\beta>3 / 2,2-\alpha-\beta<$ $\theta<\alpha+\beta-1$. Let $X$ be a Banach space, let $\Phi \in X^{*}$ and $A: \mathscr{D}(A) \subset X \rightarrow X$ and $B: \mathscr{D}(B) \subset X \rightarrow X$ be two closed linear operators such that $(i) \mathscr{D}(A) \subseteq \mathscr{D}(B)_{j}$; (ii) $A$ is invertible; (iii) $\left\|B(\lambda B+A)^{-1}\right\|_{\mathscr{L}(X)} \leq c(1+|\lambda|)^{-\beta}$ for all $\lambda \in \Sigma_{\alpha}$, where

$$
\Sigma_{\alpha}=\left\{\lambda \in \mathbb{C}: \operatorname{Re} \lambda \geq-c_{0}(1+|\lambda|)^{\alpha}\right\}, \quad c_{0}>0 .
$$

Let $g \in C^{1+\theta}([0, \tau] ; \mathbb{R})$ and $z \in X$ satisfy the following properties for some $z^{*}, v^{*} \in \mathscr{D}(A)$ :

$$
\Phi[z] \neq 0, \quad \Phi\left[B u_{0}\right]=g(0), \quad z=B z^{*}, \quad A u_{0}=B v^{*} .
$$

Then the identification problem

$$
\begin{gathered}
(B u)^{\prime}(t)+A u(t)=f(t) z, \quad 0 \leq t \leq \tau, \\
(B u)(0)=B u_{0}, \\
\Phi[B u(t)]=g(t), \quad 0 \leq t \leq \tau,
\end{gathered}
$$

admits a unique global solution:

$$
\begin{gathered}
(u, f) \in\left[C^{\theta}([0, \tau] ; \mathscr{D}(A))\right] \times C^{\theta}([0, \tau] ; \mathbb{R}), \\
B u \in C^{1+\theta}([0, \tau] ; X) .
\end{gathered}
$$

Remark 30. Assumptions (ii) and (iii) can be weakened to

(iibis) $k_{0} B+A$ is invertible for some $k_{0} \in \mathbb{R}$;

(iiibis) $\left\|B(\lambda B+A)^{-1}\right\|_{\mathscr{L}(X)} \leq c(1+|\lambda|)^{-\beta}$ for all $\lambda \in k_{0}+\Sigma_{\alpha}$.

Indeed, let us introduce the new unknown $v(t)=$ $e^{-k_{0} t} u(t)$. Then Problem (189) is equivalent to the following:

$$
\begin{gathered}
(B v)^{\prime}(t)+\left(A+k_{0} B\right) v(t)=e^{-k_{0} t} f(t) z, \quad 0 \leq t \leq \tau, \\
(B v)(0)=B u_{0}, \\
\Phi[B u(t)]=e^{-k_{0} t} g(t), \quad 0 \leq t \leq \tau .
\end{gathered}
$$

Proposition 29 applies immediately provided we replace the triplet $(A, f, g)$ by $\left(\left(A+k_{0} B\right), e^{-k_{0} t} f, e^{-k_{0} t} g\right)$. Once $v$ and $f_{1}(t)=e^{-k_{0} t} f(t)$ have been determined so are $u$ and $f$, with the same regularity.

As a consequence, we have the following result relative to the generators $-A$ of infinitely differentiable semigroups of parabolic type with nonnecessarily dense domains, satisfying

$$
\left\|(\lambda+A)^{-1}\right\|_{\mathscr{L}(X)} \leq c(1+|\lambda|)^{-\beta}, \quad \lambda \in k_{0}+\Sigma_{\alpha} .
$$

Corollary 31. Let $0<\beta \leq \alpha \leq 1, \alpha+\beta>3 / 2$, and $2-\alpha-\beta<$ $\theta<\alpha+\beta-1$. Let $\Psi \in X^{*}$ and the closed linear operator $A$ satisfy (192) in $X$. Let $g \in C^{1+\theta}([0, \tau] ; \mathbb{R}), \Phi \in X^{*}, z \in \mathscr{D}(A)$, $\Phi[z] \neq 0, w_{0} \in D\left(A^{2}\right), \Phi\left[w_{0}\right]=g(0)$. Then the identification problem

$$
\begin{gathered}
w^{\prime}(t)+A w(t)=f(t) z, \quad 0 \leq t \leq \tau, \\
w(0)=w_{0}, \\
\Psi[w(t)]=g(t), \quad 0 \leq t \leq \tau,
\end{gathered}
$$

has a unique global solution $(w, f) \in\left[C^{1+\theta}([0, \tau] ; X) \cap C^{\theta}([0\right.$, $\tau], \mathscr{D}(A))] \times C^{\theta}([0, \tau] ; \mathbb{R})$.

In particular, but very important case, where $\alpha=\beta=1$, and $X$ is reflexive, it is possible to weaken the assumptions on the initial data and $z$. Notice that in the statement of Proposition $29 z$ must belong to the range of $B A^{-1}$ (or of $\left.B\left(A+k_{0} B\right)^{-1}\right)$ and $\Phi[z] \neq 0$.

The following extension to Theorem 2.2 in [1] holds.

Proposition 32. Let $A: \mathscr{D}(A) \subset X \rightarrow X$ and $B: \mathscr{D}(B) \subset$ $X \rightarrow X$ be two closed linear operators in the reflexive Banach space $X$, with $\mathscr{D}(A) \subseteq \mathscr{D}(B), \Phi \in X^{*}$ and $g \in C^{1+\theta}([0, \tau] ; \mathbb{R})$, $\theta \in(0,1)$. Suppose

$$
\begin{gathered}
\Phi\left[B u_{0}\right]=g(0), \\
\left\|B(\lambda B+A)^{-1}\right\|_{\mathscr{L}(X)} \leq c(1+|\lambda|)^{-1}, \quad \lambda \in k_{0}+\Sigma_{\alpha} .
\end{gathered}
$$

Let $T=B\left(k_{0} B+A\right)^{-1}$ and let $P$ be the projection of $X$ on the null space $N(T)$ along $\overline{R(T)}$. Suppose $\theta_{0} \in(\theta, 1)$ and

$$
\begin{gathered}
\Phi[(I-P) z] \neq 0 \\
\sup _{t>0} t^{\theta} \|\left(A+k_{0} B\right)\left[\left(t+k_{0}\right) B+A\right]^{-1} \\
\times(I-P)\left(A+k_{0} B\right) u_{0} \| \frac{}{R(T)}<+\infty \\
\sup _{t>0} t^{\theta_{0}}\left\|\left(A+k_{0} B\right)\left[\left(t+k_{0}\right) B+A\right]^{-1}(I-P) z\right\|_{\frac{1}{R(T)}}<+\infty .
\end{gathered}
$$

Then the identification Problem (189) admits a unique global solution

$$
\begin{gathered}
(u, f) \in\left[C^{\theta}([0, \tau] ; \mathscr{D}(A))\right] \times C^{\theta}([0, \tau] ; \mathbb{R}) \\
B u \in C^{1+\theta}([0, \tau] ; X)
\end{gathered}
$$

Remark 33. If $T$ has a closed range, conditions (196)-(197) can be dropped out. Indeed, it suffices to apply Theorem 2.2 in [1]

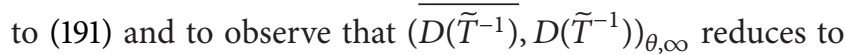
$D\left(\widetilde{T}^{-1}\right)=R(\widetilde{T})=R(T), \widetilde{T}$ denoting the restriction of $T$ to $\overline{R(T)}=R(T)$.

Remark 34. Very recently in [8, Theorem 3.1] the above results have been improved to the case $\alpha=\beta=1$ and $B=I$.

Proposition 35. Let $A$ be the generator of an analytic semigroup in the complex Banach space $X$, let $\Phi \in X^{*}, y_{0} \in$ 
$\mathscr{D}(A), A y_{0} \in(X, \mathscr{D}(A))_{\theta, \infty}$ for some $\theta \in(0,1)$, and let $g \in$ $C^{1+\theta}([0, \tau] ; \mathbb{C}), z \in(X, \mathscr{D}(A))_{\theta, \infty}, \Phi[z] \neq 0, \Phi\left[y_{0}\right]=g(0)$. Then the inverse problem

$$
\begin{gathered}
y^{\prime}(t)=A y(t)+f(t) z, \quad 0 \leq t \leq \tau, \\
y(0)=y_{0}, \\
\Phi[y(t)]=g(t), \quad 0 \leq t \leq \tau,
\end{gathered}
$$

admits a unique solution $(y, f)$ such that

$$
\begin{gathered}
t \in C^{1+\theta}([0, \tau] ; X) \cap C^{\theta}([0, \tau] ; \mathscr{D}(A)) \\
f \in C^{\theta}([0, \tau] ; \mathbb{R})
\end{gathered}
$$

As for Problem (199), also the hyperbolic case, corresponding to the case where A generates a $C_{0}$-semigroup, has been dealt with in [8, Corollary 2.1].

Proposition 36. Let $A$ be the generator of a $C_{0}$-semigroup in the complex Banach space $X$, and let $\Phi \in X^{*}, z \in \mathscr{D}(A)$, $\Phi[z] \neq 0, y_{0} \in \mathscr{D}(A), g \in C^{1}([0, \tau] ; \mathbb{C}), \Phi\left[y_{0}\right]=g(0)$. Then the inverse Problem (199) admits a unique solution $(y, f) \in$ $\left[C^{1}([0, \tau] ; X) \cap C([0, \tau] ; \mathscr{D}(A))\right] \times C([0, \tau] ; \mathbb{R})$.

In the next section, we are giving specific applications of the results listed in this section.

\section{Applications}

In this section, we will give several concrete applications of our previous abstract results.

Problem 37. First, we recall some previous results from [1517]. Let $L$ and $M$ be two linear differential operators with domains in $L^{p}(\Omega), p \in(1,+\infty), \Omega \in \mathbb{R}^{n}$ being a bounded region with a boundary $\partial \Omega$ of class $C^{2} . M-$ a multiplication operator by a nonnegative function $m \in L^{\infty}(\Omega)$-is defined by

$$
\mathscr{D}(M)=L^{p}(\Omega), \quad M u(x)=m(x) u(x), \quad x \in \Omega .
$$

Operator $L$ is defined either by

$$
\begin{gathered}
\mathscr{D}(L)=W^{2, p}(\Omega) \cap W_{0}^{1, p}(\Omega), \\
L u(x)=\mathscr{L} u(x) \\
:=-\sum_{i, j=1}^{n} D_{x_{i}}\left[a_{i, j}(x) D_{x_{j}} u(x)\right] \\
+\sum_{j=1}^{n} a_{j}(x) D_{x_{j}} u(x)+a_{0}(x) u(x), \\
\quad x \in \Omega, \quad u \in \mathscr{D}(L),
\end{gathered}
$$

or by

$\mathscr{D}(L)$

$$
\begin{gathered}
=\left\{u \in W^{2, p}(\Omega): \sum_{i, j=1}^{n} a_{i, j}(x) v_{i}(x) D_{x_{j}} u(x)\right. \\
+b(x) u(x)=0 \text { on } \partial \Omega\}, \\
\operatorname{Lu}(x)=\mathscr{L} u(x), \quad x \in \Omega, u \in \mathscr{D}(L) .
\end{gathered}
$$

We assume that the coefficients $a_{i, j}, a_{j}, a_{0}$ enjoy the following properties:

$$
\begin{gathered}
a_{i, j}, D_{x_{i}} a_{i, j}, a_{i}, D_{x_{i}} a_{i}, a_{0} \in C(\bar{\Omega}), \quad i, j=1, \ldots, n, \\
a_{i, j}(x)=a_{j, i}(x), \quad x \in \bar{\Omega}, \\
\sum_{i, j=1}^{n} a_{i, j}(x) \xi_{i} \xi_{j} \geq c_{0}|\xi|^{2}, \quad x \in \bar{\Omega}, \quad \xi \in \mathbb{R}^{n}, \\
a_{0}(x)-\frac{1}{p} \sum_{i=1}^{n} D_{x_{i}} a_{i}(x) \geq c_{1}, \quad x \in \bar{\Omega}, \\
b(x)+\frac{1}{p} \sum_{i=1}^{n} a_{i}(x) v_{i}(x) \geq 0, \quad x \in \partial \Omega,
\end{gathered}
$$

$c_{0}$ and $c_{1}$ being two positive constants.

Then it is shown in Theorem $2.1 \mathrm{in}$ [17] that the pair $(L, M)$ satisfies in the sector

$$
\Sigma(c)=\{z \in \mathbb{C}: \operatorname{Re} z \geq c(1+|z|)\}, \quad c \in \mathbb{R}_{+}
$$

the following estimate with $\beta=1 / p$ :

$$
\left\|M(z M+L)^{-1}\right\|_{\mathscr{L}(X)} \leq C(1+|z|)^{-\beta} .
$$

Let us consider the following identification parabolic-elliptic problem:

$$
\begin{aligned}
& D_{t}\left[\begin{array}{cc}
m_{1}(x) & k m_{2}(x)^{b} \\
0 & m_{2}(x)^{a}
\end{array}\right]\left[\begin{array}{l}
u(t, x) \\
v(t, x)
\end{array}\right] \\
& +\left[\begin{array}{cc}
L_{1}\left(x, D_{x}\right) & L_{2}\left(x, D_{x}\right) \\
O & L_{4}\left(x, D_{x}\right)
\end{array}\right]\left[\begin{array}{l}
u(t, x) \\
v(t, x)
\end{array}\right] \\
& =f(t)\left[\begin{array}{l}
z_{1} \\
z_{1}
\end{array}\right], \quad \forall(t, x) \in[0, \tau] \times \Omega, \\
& {\left[\begin{array}{cc}
m_{1}(x) & k m_{2}(x)^{b} \\
0 & m_{2}(x)^{a}
\end{array}\right]\left[\begin{array}{c}
u(0, x) \\
v(0, x)
\end{array}\right]} \\
& =\left[\begin{array}{cc}
m_{1}(x) & k m_{2}(x)^{b} \\
0 & m_{2}(x)^{a}
\end{array}\right]\left[\begin{array}{l}
u_{0}(x) \\
v_{0}(x)
\end{array}\right], \quad \forall x \in \Omega, \\
& \int_{\Omega}\left\{\begin{array}{l}
\eta_{1}(x)\left[m_{1}(x) u(t, x)+k m_{2}(x)^{b} v(t, x)\right] \\
\left.+\eta_{2}(x) m_{2}(x)^{a} v(t, x)\right\} d x=g(t), \quad \forall t \in[0, \tau],
\end{array}\right.
\end{aligned}
$$


where the linear operators $L_{i}\left(x, D_{x}\right), i=1,2,4$, and $\mathscr{D}\left(L_{4}\right) \subset$ $\mathscr{D}\left(L_{2}\right)$ enjoy the same properties as $L\left(x, D_{x}\right)$ with $\beta=1 / p$, $m_{1}, m_{2} \in L^{\infty}(\Omega)$ with $m_{1} \geq 0$ and $m_{2} \geq 0$ a.e. in $\Omega, k \neq 0$ (if $k=0$ we can apply Remark 19), $0<a \leq b, z_{1}, z_{2}, u_{0}, v_{0} \in$ $L^{p}(\Omega), \eta_{1}, \eta_{2} \in L^{q}(\Omega), 1 / p+1 / q=1, g \in C([0, \tau] ; \mathbb{R})$.

We know that the pairs $\left(L_{1}\left(x, D_{x}\right), M_{1}\right),\left(L_{2}\left(x, D_{x}\right), M_{4}\right)$, where $M_{4} u(x)=m_{2}(x)^{a} u(x)$, satisfy $(206)$ in $L^{p}(\Omega)$.

In view of the moment inequality (cfr. [18, page 115]), assumption (97) holds with $\delta=b / a$. Therefore, Theorem 14 applies provided that $(2 / p)+(b / a)>5 / 2$; that is, $p<$ $((5 a-2 b) / 4 a)^{-1}$. So, we must necessarily have $b \leq a<2 b$ and $1<p<4 / 3$ respectively.

Let $\theta \in(3-(2 / p)-(b / a),(2 / p)+(b / a)-2), 1<p<$ $4 a /(5 a-2 b)$,

$$
\begin{gathered}
{\left[\begin{array}{c}
z_{1} \\
z_{2}
\end{array}\right]=\left[\begin{array}{c}
m_{1} z_{1}^{*}+k m_{2}^{b} z_{2}^{*} \\
m_{2}^{a} z_{2}^{*}
\end{array}\right],} \\
{\left[\begin{array}{c}
L_{1}\left(\cdot, D_{x}\right) u_{0}+L_{2}\left(\cdot, D_{x}\right) v_{0} \\
L_{4}\left(\cdot, D_{x}\right) v_{0}
\end{array}\right]=\left[\begin{array}{c}
m_{1} u_{0}^{*}+k m_{2}^{b} v_{0}^{*} \\
m_{2}^{a} v_{0}^{*}
\end{array}\right],}
\end{gathered}
$$

where $z_{1}^{*}, u_{0}^{*} \in \mathscr{D}\left(L_{1}\right), z_{2}^{*}, v_{0}^{*} \in \mathscr{D}\left(L_{4}\right)$,

$$
\begin{gathered}
\int_{\Omega}\left[\eta_{1}(x) z_{1}(x)+\eta_{2}(x) z_{2}(x)\right] d x \neq 0, \\
\int_{\Omega}\left\{\eta_{1}(x)\left[m_{1}(x) u_{0}(x)+k m_{2}(x)^{b} v_{0}(x)\right]\right. \\
\left.+\eta_{2}(x) m_{2}(x)^{a} v_{0}(x)\right\} d x=g(0) .
\end{gathered}
$$

Then Problem (207) admits a unique global solution $(u, v$, f) $\in C^{\theta}\left([0, \tau] ; \mathscr{D}\left(L_{1}\right)\right) \times C^{\theta}\left([0, \tau] ; \mathscr{D}\left(L_{4}\right)\right) \times C^{\theta}([0, \tau] ; \mathbb{R})$, $m_{1} u+k m_{2}^{b} v, m_{2}^{a} v \in C^{1+\theta}\left([0, \tau] ; L^{p}(\Omega)\right)$.

Using the same scheme, one could handle the more general problem:

$$
\begin{aligned}
D_{t} & {\left[\begin{array}{cc}
m_{1}(x) & k m_{2}(x)^{b} \\
0 & m_{2}(x)^{a}
\end{array}\right]\left[\begin{array}{l}
u(t, x) \\
v(t, x)
\end{array}\right] } \\
& +\left[\begin{array}{ll}
L_{1}\left(x, D_{x}\right) & L_{2}\left(x, D_{x}\right) \\
k_{1} m_{1}(x)^{c} & L_{4}\left(x, D_{x}\right)
\end{array}\right]\left[\begin{array}{l}
u(t, x) \\
v(t, x)
\end{array}\right] \\
\quad & f(t)\left[\begin{array}{l}
z_{1} \\
z_{2}
\end{array}\right], \quad \forall(t, x) \in[0, \tau] \times \Omega,
\end{aligned}
$$

where $c \in(0,1], k_{1}$ is constant, provided that $(2 / p)+(b / a)+$ $c>3$ (cf. Theorem 14).

As a particular case, we can also treat the problem

$$
\begin{aligned}
& D_{t}\left[\begin{array}{cc}
m_{1}(x) & m_{2}(x)^{b} \\
0 & m_{2}(x)^{a}
\end{array}\right]\left[\begin{array}{l}
u(t, x) \\
v(t, x)
\end{array}\right] \\
& +\left[\begin{array}{cc}
L_{1}\left(x, D_{x}\right) & n_{1}(x) \\
k m_{1}(x)^{c} & n_{2}(x)
\end{array}\right]\left[\begin{array}{l}
u(t, x) \\
v(t, x)
\end{array}\right] \\
& \quad=f(t)\left[\begin{array}{l}
z_{1} \\
z_{2}
\end{array}\right], \quad \forall(t, x) \in[0, \tau] \times \Omega,
\end{aligned}
$$

where $n_{1}, n_{2} \in C(\bar{\Omega} ; \mathbb{R}), n_{2}(x) \geq \tilde{c}>0, c \in(0,1], k \in \mathbb{R}$.
Problem 37bis. We show here how some more regularity of functions $m_{1}$ and $m_{2}$ allow to choose a larger exponent $p$ in the reference space $L^{p}(\Omega)$.

We recall that a function $m \in C^{1}(\bar{\Omega}), m(x) \geq 0, x \in \bar{\Omega}$, is said to be $\rho$-regular for some $\rho \in(0,1][3,15,16]$ if there exists a positive constant $C$ such that

$$
|\nabla m(x)| \leq C m(x)^{\rho}, \quad x \in \bar{\Omega} .
$$

If $m_{0} \in C^{1}(\bar{\Omega}), m_{0}(x) \geq 0, x \in \bar{\Omega}$, and $k>1$, then $m(x)=$ $m_{0}(x)^{k}$ is $\rho$-regular with $\rho=(k-1) / k$.

If $m$ is $\rho$-regular and

$$
\begin{gathered}
\sum_{i=1}^{n} a_{i}(x) D_{x_{i}} m(x) \leq 0, \quad x \in \bar{\Omega}, \\
b(x)+\frac{1}{p} m(x)^{p-1} \sum_{i=1}^{n} a_{i}(x) v_{i}(x) \geq 0, \quad x \in \bar{\Omega},
\end{gathered}
$$

then (cfr. Theorem 3.3 in [17]) the pair $(L, M)$ satisfies (206) with $\alpha=1, \beta=2[p(2-\rho)]^{-1}$. Note that $\beta>1 / 2$ if and only if $p \in\left[2,4(2-\rho)^{-1}\right)$.

Let now $m_{1}$ be $\rho_{1}$-regular, so that $\left(L_{1}\left(x, D_{x}\right), M_{1}\right)$ satisfies (206) with $\beta_{1}=2\left[p\left(2-\rho_{1}\right)\right]^{-1}$ according to [15]. Moreover, let $m_{2} \in C^{1}(\bar{\Omega}), m_{2}(x) \geq 0, x \in \bar{\Omega}, a>1$. Then, $\beta_{4}=2[p(1+$ $(1 / a))]^{-1}$. Thus, we must have

$$
\frac{2}{p}\left(\frac{1}{2-\rho_{1}}+\frac{a}{1+a}\right)+\frac{b}{a}>\frac{5}{2}, \quad \frac{b}{a}>\frac{1}{2}
$$

to find

$$
\begin{aligned}
2 & \leq p<\frac{4 a}{5 a-2 b}\left(\frac{1}{2-\rho_{1}}+\frac{a}{1+a}\right) \\
& \Longrightarrow \frac{2 a}{5 a-2 b}\left(\frac{1}{2-\rho_{1}}+\frac{a}{1+a}\right)>1
\end{aligned}
$$

Let $b=v a, 1 / 2<v<1$. Then the right-hand side in (215) changes to

$$
\frac{1}{2-\rho_{1}}+\frac{b}{b+v}+v>\frac{5}{2}
$$

In particular, the last property is achieved if $\rho_{1}$ tends to 1 and $a$ is large enough.

Therefore, Theorem 14 applies in the reference space $L^{p}(\Omega)$ with $b=v a$ and

$$
2 \leq p<\frac{4}{5-2 \nu}\left(\frac{1}{2-\rho_{1}}+\frac{a}{1+a}\right)
$$

whenever the following inequalities hold:

$$
\frac{1}{2-\rho_{1}}+\frac{a}{1+a}+v>\frac{5}{2}, \quad \frac{1}{2}<v<1 .
$$


Problem 38. We are concerned with the initial and boundary value problem:

$$
\begin{aligned}
& D_{t}\left(\left[\begin{array}{ll}
m_{1}(x) & m_{2}(x) \\
m_{3}(x) & m_{4}(x)
\end{array}\right]\left[\begin{array}{l}
u(t, x) \\
v(t, x)
\end{array}\right]\right) \\
& +\left[\begin{array}{ll}
\alpha_{1}(x) L_{1}\left(x, D_{x}\right) & \alpha_{2}(x) L_{2}\left(x, D_{x}\right) \\
\alpha_{3}(x) L_{3}\left(x, D_{x}\right) & \alpha_{4}(x) L_{2}\left(x, D_{x}\right)
\end{array}\right]\left[\begin{array}{l}
u(t, x) \\
v(t, x)
\end{array}\right] \\
& =f(t)\left[\begin{array}{l}
z_{1} \\
z_{1}
\end{array}\right], \quad \forall(t, x) \in[0, \tau] \times \Omega, \\
& {\left[\begin{array}{ll}
m_{1}(x) & m_{2}(x) \\
m_{3}(x) & m_{4}(x)
\end{array}\right]\left[\begin{array}{l}
u(0, x) \\
v(0, x)
\end{array}\right]} \\
& =\left[\begin{array}{ll}
m_{1}(x) & m_{2}(x) \\
m_{3}(x) & m_{4}(x)
\end{array}\right]\left[\begin{array}{l}
u_{0}(x) \\
v_{0}(x)
\end{array}\right], \quad x \in \Omega, \\
& \int_{\Omega}\left\{\eta_{1}(x)\left[m_{1}(x) u(t, x)+m_{2}(x) v(t, x)\right]\right. \\
& \left.+\eta_{2}(x)\left[m_{3}(x) u(t, x)+m_{4}(x) v(t, x)\right]\right\} d x \\
& =g(t), \quad 0 \leq t \leq \tau,
\end{aligned}
$$

where for the sake of simplicity, we have set

$$
L_{j}\left(x, D_{x}\right)=-\Delta+a_{j}(x), \quad j=1,2,3
$$

and have endowed such operators with either the Dirichlet or the Robin boundary condition.

We will assume that $m_{j} \in L^{\infty}(\Omega), j=1, \ldots, 4$, are realvalued, while $\alpha_{j} \in C(\bar{\Omega}), j=1, \ldots, 4$, and $a_{i} \in C(\bar{\Omega})$, $j=1,2,3$, are scalar functions, the $a_{i}$ 's being nonnegative, satisfying the following properties:

$$
\begin{gathered}
m_{j}(x) \geq C_{j}>0, \quad j=1,3, \\
-C\left(\frac{m_{1}(x)}{m_{3}(x)} m_{4}(x)-m_{2}(x)\right) \leq m_{2}(x) \\
\leq \frac{m_{1}(x)}{m_{3}(x)} m_{4}(x), \quad x \in \Omega, \\
\alpha_{1} \geq c>0, \quad \alpha_{2}(x)-\frac{m_{1}(x)}{m_{3}(x)} \alpha_{4}(x) \leq-\bar{c}<0, \\
\alpha_{3}(x)=\frac{m_{1}(x)}{m_{3}(x)} \alpha_{1}(x), \quad x \in \Omega .
\end{gathered}
$$

We observe that properties (221) hold if $m_{2}(x) \leq 0$ and $m_{4}(x) \geq 0$ for all $x \in \bar{\Omega}$. This choice implies $C=1$ and $\delta=1$ in (97). Moreover, the last condition in (222) implies (137) and $\delta_{1}=1$.

Assume now that the matrix operator

$$
\left[\begin{array}{ll}
\alpha_{1}(x) L_{1}\left(x, D_{x}\right) & \alpha_{2}(x) L_{2}\left(x, D_{x}\right) \\
\alpha_{3}(x) L_{3}\left(x, D_{x}\right) & \alpha_{4}(x) L_{2}\left(x, D_{x}\right)
\end{array}\right]
$$

is invertible in $L^{p}(\Omega) \times L^{p}(\Omega), p \in(1,+\infty)$. For this purpose, it is enough to assume

$$
\begin{gathered}
\left|\alpha_{1}(x) \alpha_{4}(x)-\alpha_{2}(x) \alpha_{3}(x)\right| \geq C_{0}>0, \\
\frac{\alpha_{1}(x) \alpha_{4}(x) a_{1}(x)-\alpha_{2}(x) \alpha_{3}(x) a_{2}(x)}{\alpha_{1}(x) \alpha_{4}(x)-\alpha_{2}(x) \alpha_{3}(x)} \geq 0,
\end{gathered}
$$

for all $x \in \Omega$. Further, $z_{1}, z_{2}, \eta_{1}, \eta_{2}$ are given functions on $\Omega$.

Relying on the proof of Theorem 18 we are led to the following equation:

$$
\begin{aligned}
& D_{t}\left(\left[\begin{array}{cc}
m_{1}(x) & m_{2}(x) \\
0 & -\frac{m_{1}(x)}{m_{3}(x)} m_{4}(x)+m_{2}(x)
\end{array}\right]\left[\begin{array}{l}
u(t, x) \\
v(t, x)
\end{array}\right]\right) \\
& +\left[\begin{array}{cc}
\alpha_{1}(x) L_{1}\left(x, D_{x}\right) & \alpha_{2}(x) L_{2}\left(x, D_{x}\right) \\
-\frac{m_{1}(x)}{m_{3}(x)} \alpha_{3}(x) L_{3}\left(x, D_{x}\right)+\alpha_{1}(x) L_{1}\left(x, D_{x}\right) & \left(\alpha_{2}(x)-\frac{m_{1}(x)}{m_{3}(x)} \alpha_{4}(x)\right) L_{2}\left(x, D_{x}\right)
\end{array}\right] \\
& \times\left[\begin{array}{l}
u(t, x) \\
v(t, x)
\end{array}\right] \\
& =f(t)\left[\begin{array}{c}
z_{1}(x) \\
z_{1}(x)-\frac{m_{1}(x)}{m_{3}(x)} z_{2}(x)
\end{array}\right], \quad \forall(t, x) \in[0, \tau] \times \Omega .
\end{aligned}
$$


Under our assumptions (221)-(224) Theorem 18 applies with $\alpha=\delta=\beta_{1}=1, \beta_{4}=1 / p>1 / 2$, that is, $p \in(1,2)$, if the following additional properties are satisfied when $z_{1}^{*}, u_{0}^{*} \in \mathscr{D}\left(L_{1}\right), z_{2}^{*}, v_{0}^{*} \in \mathscr{D}\left(L_{2}\right), g \in C^{1+\theta}([0, \tau] ; \mathbb{R})$, with $\theta \in\left(1 / p^{\prime}, 1 / p\right), \eta_{1}, \eta_{2} \in L^{p^{\prime}}(\Omega)$ :

$$
\begin{gathered}
{\left[\begin{array}{l}
z_{1}(x) \\
z_{2}(x)
\end{array}\right]=\left[\begin{array}{l}
m_{1}(x) z_{1}^{*}(x)+m_{2}(x) z_{2}^{*}(x) \\
m_{3}(x) z_{1}^{*}(x)+m_{4}(x) z_{2}^{*}(x)
\end{array}\right],} \\
{\left[\begin{array}{l}
\alpha_{1}(x) L_{1}\left(x, D_{x}\right) u_{0}(x)+\alpha_{2}(x) L_{2}\left(x, D_{x}\right) v_{0}(x) \\
\alpha_{3}(x) L_{3}\left(x, D_{x}\right) u_{0}(x)+\alpha_{4}(x) L_{2}\left(x, D_{x}\right) v_{0}(x)
\end{array}\right]} \\
=\left[\begin{array}{l}
m_{1}(x) u_{0}^{*}(x)+m_{2}(x) v_{0}^{*}(x) \\
m_{3}(x) u_{0}^{*}(x)+m_{4}(x) v_{0}^{*}(x)
\end{array}\right], \\
\int_{\Omega}\left\{\eta_{1}(x) z_{1}(x)+\eta_{2}(x) z_{2}(x)\right\} d x \neq 0, \\
\int_{\Omega}\left\{\eta_{1}(x)\left[m_{1}(x) u_{0}(x)+m_{2}(x) v_{0}(x)\right]\right. \\
\left.+\eta_{2}(x)\left[m_{3}(x) u_{0}(x)+m_{4}(x) v_{0}(x)\right]\right\} d x=g(0) .
\end{gathered}
$$

Then the identification Problems (221)-(224) admit a unique global strict solution $(u, v, f) \in C^{\theta}\left([0, \tau] ; \mathscr{D}\left(L_{1}\right)\right) \times C^{\theta}([0$, $\left.\tau] ; \mathscr{D}\left(L_{2}\right)\right) \times C^{\theta}([0, \tau] ; \mathbb{R})$ such that $m_{1+2 j} u+m_{2+2 j} v \in$ $C^{1+\theta}\left([0, \tau] ; L^{p}(\Omega)\right), j=0,1$ and $p \in(1,2)$.

We observe that if $m_{j}, \alpha_{j}, j=1, \ldots, 4$, and $a_{i}, i=1,2,3$, are more regular, the previous result can be extended to the case where $p \in[2,+\infty)$ and $\beta_{4}$ is larger. For this purpose, we assume that $m:=m_{1} m_{4}-m_{2} m_{3} \geq 0$ is $\rho$-regular; that is,

$$
m \in C^{1}(\bar{\Omega}), \quad|\nabla m(x)| \leq c m(x)^{\rho}, \quad x \in \bar{\Omega}, \rho \in(0,1] .
$$

Since $n(x)=\alpha_{4}(x)\left(m_{1}(x) / m_{3}(x)\right)-\alpha_{2}(x) \geq \bar{c}>0$ for all $x \in \bar{\Omega}$, we get

$$
\begin{array}{r}
\left|D_{x_{j}}\left(\frac{m(x)}{n(x)}\right)\right|=n(x)^{-2}\left|n(x) D_{x_{j}} m(x)-m(x) D_{x_{j}} n(x)\right| \\
\leq C \frac{m(x)^{\rho}}{n(x)}+\frac{m(x)}{n(x)} \frac{D_{x_{j}} n(x)}{n(x)} \leq C^{\prime}\left(\frac{m(x)}{n(x)}\right)^{\rho}, \\
x \in \bar{\Omega} .
\end{array}
$$

Therefore, owing to Theorem 3.3 in [15], we can choose $\beta_{4}=$ $2[p(2-\rho)]^{-1}, p \in[2,+\infty)$. This choice implies $\beta_{4}>1 / 2$ and $p \in\left[2,4(2-\rho)^{-1}\right)$, so that in this case $\theta$ runs in the interval $\left(1-2[p(2-\rho)]^{-1}, 2[p(2-\rho)]^{-1}\right)$.

In the case when $p \in(1,2)$ and $m$ is $\rho$-regular with $\rho \in$ $(2-p, 1)$, then, owing to Theorem 4.2 in [15], we can choose $\beta_{4}=[p(2-\rho)]^{-1}$ so that $\theta \in\left((1-\rho)(2-\rho)^{-1},(2-\rho)^{-1}\right)$.

Problem 38bis. We could handle also the case where the determinant $m_{1}(x) m_{4}(x)-m_{2}(x) m_{3}(x)$ vanishes everywhere in $\bar{\Omega}$. However, in this case, Theorem 18 forces us to assume $m_{2}(x)=0$ for all $x \in \bar{\Omega}$.

In view of assumptions (222) the second equation in system (225) becomes

$$
\begin{aligned}
& {\left[\frac{m_{1}(x)}{m_{3}(x)} \alpha_{4}(x)-\alpha_{1}(x)\right] L_{2}(x, D) v(t, x)} \\
& \quad=f(t)\left[\frac{m_{1}(x)}{m_{3}(x)} z_{2}(x)-z_{1}(x)\right] \\
& \quad-\left[\frac{m_{1}(x)}{m_{3}(x)} \alpha_{3}(x) a_{3}(x)-\alpha_{1}(x) a_{1}(x)\right] u(t, x)
\end{aligned}
$$

Thus all is reduced to a regular identification problem.

However, using Theorem 23, in some cases we can handle the situation when $m_{2} \not \equiv 0$. For the sake of simplicity we choose $L_{1}=L_{2}=L_{3}=L$ and assume $\alpha_{4} / \alpha_{3}=\alpha_{2} / \alpha_{1}=\nu=$ const., so that

for some constant $v \neq 0$. Therefore our differential equation becomes

$$
\begin{aligned}
& D_{t}\left(\left[\begin{array}{cc}
m_{1}(x) & m_{2}(x) \\
0 & 0
\end{array}\right]\left[\begin{array}{l}
u(t, x) \\
v(t, x)
\end{array}\right]\right) \\
& \quad+\left[\left(\alpha_{1}(x)-\frac{m_{1}(x)}{m_{3}(x)} \alpha_{3}(x)\right) L\left(x, D_{x}\right)\left(\alpha_{2}(x)-\frac{m_{1}(x)}{m_{3}(x)} \alpha_{4}(x)\right) L\left(x, D_{x}\right)\right]\left[\begin{array}{c}
u(t, x) \\
v(t, x)
\end{array}\right] \\
& \quad=f(t)\left[\begin{array}{c}
z_{1}(x) \\
z_{1}(x)-\frac{m_{1}(x)}{m_{3}(x)} z_{2}(x)
\end{array}\right], \quad \forall(t, x) \in[0, \tau] \times \Omega .
\end{aligned}
$$


Referring to the notation in Theorem 23, we get

$$
\begin{gathered}
S=M_{1}-M_{2} L_{4}^{-1} L_{3}=\left(m_{1}-v^{-1} m_{2}\right) I, \\
L_{1}-L_{2} L_{4}^{-1} L_{3}=\left(\alpha_{1}-v^{-1} \alpha_{2}\right) L(\cdot, D), \\
\mathbb{A}=\left(\alpha_{1}-v^{-1} \alpha_{2}\right) L(\cdot, D)\left(m_{1}-v^{-1} m_{2}\right)^{-1} .
\end{gathered}
$$

Consequently, the linear closed operator $-\mathbb{A}$ generates an analytic semigroup of linear bounded operators provided $\alpha_{1}(x)-v^{-1} \alpha_{2}(x)>0$ and $m_{1}(x)-v^{-1} m_{2}(x)>0$ for all $x \in \bar{\Omega}$. In this case the resolvent of $-\mathbb{A}$ admits the representation:

$$
\begin{aligned}
(\lambda+\mathbb{A})^{-1}= & \left(m_{1}-v^{-1} m_{2}\right) \\
& \times\left[\lambda\left(m_{1}-v^{-1} m_{2}\right)\left(\alpha_{1}-\nu^{-1} \alpha_{2}\right)^{-1}+\mathbb{A}\right]^{-1} \\
& \times\left(\alpha_{1}-v \alpha_{2}\right)^{-1}
\end{aligned}
$$

The element $\bar{z}$ in Theorem 23 can be easily described.

Example 39. Let $\Omega=B(0,1)=\left\{x \in \mathbb{R}^{n}:\|x\|<1\right\}$ and let operator $L(x, D)=-\Delta+a(x)$ be endowed with either Dirichlet or Robin boundary conditions.

Consider then the following identification problem, where $r$ and $s$ are real positive numbers:

$$
\begin{aligned}
& D_{t}\left(\left[\begin{array}{cc}
1 & -\left(1-\|x\|^{2}\right)^{r} \\
1 & \left(1-\|x\|^{2}\right)^{s}
\end{array}\right]\left[\begin{array}{l}
u(t, x) \\
v(t, x)
\end{array}\right]\right) \\
& +\left[\begin{array}{ll}
\alpha_{1}(x) L\left(x, D_{x}\right) & \alpha_{2}(x) L\left(x, D_{x}\right) \\
\alpha_{1}(x) L\left(x, D_{x}\right) & \alpha_{4}(x) L\left(x, D_{x}\right)
\end{array}\right]\left[\begin{array}{l}
u(t, x) \\
v(t, x)
\end{array}\right] \\
& =f(t)\left[\begin{array}{l}
z_{1}(x) \\
z_{2}(x)
\end{array}\right], \quad \forall(t, x) \in[0, \tau] \times \Omega, \\
& {\left[\begin{array}{cc}
1 & -\left(1-\|x\|^{2}\right)^{r} \\
1 & \left(1-\|x\|^{2}\right)^{s}
\end{array}\right]\left[\begin{array}{l}
u(0, x) \\
v(0, x)
\end{array}\right]} \\
& =\left[\begin{array}{cc}
1 & -\left(1-\|x\|^{2}\right)^{r} \\
1 & \left(1-\|x\|^{2}\right)^{s}
\end{array}\right]\left[\begin{array}{l}
u_{0}(x) \\
v_{0}(x)
\end{array}\right], \quad x \in \Omega, \\
& \int_{\Omega}\left\{\eta_{1}(x)\left[u(t, x)-\left(1-\|x\|^{2}\right)^{r} v(t, x)\right]\right. \\
& \left.+\eta_{2}(x)\left[u(t, x)+\left(1-\|x\|^{2}\right)^{s} v(t, x)\right]\right\} d x=g(t) \\
& 0 \leq t \leq \tau .
\end{aligned}
$$

If we suppose that

$$
\begin{array}{cl}
\alpha_{1}, \alpha_{2}, \alpha_{4} \in C(\bar{\Omega}) \cap C^{1}(\Omega), & \alpha_{1}(x) \geq C>0, \\
\alpha_{2}(x)-\alpha_{4}(x)<0, \quad x \in \bar{\Omega}, &
\end{array}
$$

all our conditions in the last lines in Problem 37 are verified for $\beta_{4}=1 / p, p \in(1,2)$. Moreover, if $r, s \in(1,+\infty]$, then $m(x)=\left(1-\|x\|^{2}\right)^{r}+\left(1-\|x\|^{2}\right)^{s}$ belongs to $C^{1}(\bar{\Omega})$ and from the inequality $\nabla\left(1-\|x\|^{2}\right)^{r} \leq C\left[\left(1-\|x\|^{2}\right)^{r}\right]^{(r-1) / r}$ we deduce

$$
\begin{aligned}
|\nabla m(x)|=\left|\nabla\left[\left(1-\|x\|^{2}\right)^{r}+\left(1-\|x\|^{2}\right)^{s}\right]\right| \\
\leq C\left\{\left[\left(1-\|x\|^{2}\right)^{r}\right]^{(r-1) / r}+\left[\left(1-\|x\|^{2}\right)^{s}\right]^{(s-1) / s}\right\} \\
\leq C\left\{\left[\left(1-\|x\|^{2}\right)^{r}+\left(1-\|x\|^{2}\right)^{s}\right]^{(r-1) / r}\right. \\
\left.+\left[\left(1-\|x\|^{2}\right)^{r}+\left(1-\|x\|^{2}\right)^{s}\right]^{(s-1) / s}\right\} \\
\leq m(x)^{(\min (r, s)-1) / \min (r, s)}, \quad x \in \bar{\Omega} .
\end{aligned}
$$

That is, $m$ is $\rho$-regular with $\rho=(\min (r, s)-1) / \min (r, s)$, and the arguments in the treatment of Problem 38 apply as well.

Example 40. Consider the identification problem:

$$
\begin{aligned}
& D_{t}\left(\left[\begin{array}{cc}
m(x)^{2} & m(x) n(x) \\
m(x) n(x) & n(x)^{2}
\end{array}\right]\left[\begin{array}{l}
u(t, x) \\
v(t, x)
\end{array}\right]\right) \\
& +\left[\begin{array}{ll}
\alpha_{1}(x) L\left(x, D_{x}\right) & \alpha_{2}(x) L\left(x, D_{x}\right) \\
\alpha_{3}(x) L\left(x, D_{x}\right) & \alpha_{4}(x) L\left(x, D_{x}\right)
\end{array}\right]\left[\begin{array}{l}
u(t, x) \\
v(t, x)
\end{array}\right] \\
& \quad=f(t)\left[\begin{array}{l}
z_{1}(x) \\
z_{2}(x)
\end{array}\right], \quad \forall(t, x) \in[0, \tau] \times \Omega, \\
& {\left[\begin{array}{cc}
m(x)^{2} & m(x) n(x) \\
m(x) n(x) & n(x)^{2}
\end{array}\right]\left[\begin{array}{l}
u(0, x) \\
v(0, x)
\end{array}\right]} \\
& =\left[\begin{array}{cc}
m(x)^{2} & m(x) n(x) \\
m(x) n(x) & n(x)^{2}
\end{array}\right]\left[\begin{array}{l}
u_{0}(x) \\
v_{0}(x)
\end{array}\right], \\
& \int_{\Omega} \eta(x)\left[m(x)^{2} u(t, x)+m(x) n(x) v(t, x)\right] d x=g(t),
\end{aligned}
$$

where $m(x) \geq c>0$ and $n(x) \geq c>0$ for all $x \in \bar{\Omega}$. Observe that the determinant of the matrix inside the time derivative vanishes identically in $\bar{\Omega}$. 
As in the proof of Theorem 18 we obtain the equivalent problem:

$$
\begin{aligned}
& D_{t}\left(\left[\begin{array}{cc}
m(x)^{2} & m(x) n(x) \\
0 & 0
\end{array}\right]\left[\begin{array}{l}
u(t, x) \\
v(t, x)
\end{array}\right]\right) \\
& +\left[\begin{array}{cc}
\alpha_{1}(x) L\left(x, D_{x}\right) & \alpha_{2}(x) L\left(x, D_{x}\right) \\
\left(\alpha_{1}(x)-\frac{m(x)}{n(x)} \alpha_{3}(x)\right) L\left(x, D_{x}\right) & \left(\alpha_{2}(x)-\frac{m(x)}{n(x)} \alpha_{4}(x)\right) L\left(x, D_{x}\right)
\end{array}\right]\left[\begin{array}{l}
u(t, x) \\
v(t, x)
\end{array}\right] \\
& =f(t)\left[\begin{array}{c}
z_{1}(x) \\
z_{1}(x)-\frac{m(x)}{n(x)} z_{2}(x)
\end{array}\right], \quad \forall(t, x) \in[0, \tau] \times \Omega, \\
& {\left[\begin{array}{cc}
m(x)^{2} & m(x) n(x) \\
0 & 0
\end{array}\right]\left[\begin{array}{l}
u(0, x) \\
v(0, x)
\end{array}\right]=\left[\begin{array}{cc}
m(x)^{2} & m(x) n(x) \\
0 & 0
\end{array}\right]\left[\begin{array}{l}
u_{0}(x) \\
v_{0}(x)
\end{array}\right], \quad \forall x \in \Omega,} \\
& \int_{\Omega} \eta(x)\left[m(x)^{2} u(t, x)+m(x) n(x) v(t, x)\right] d x=g(t), \quad 0 \leq t \leq \tau .
\end{aligned}
$$

If

$$
\frac{m \alpha_{3}-n \alpha_{1}}{m \alpha_{4}-n \alpha_{2}}=v=\text { const., }
$$

then $\mathbb{A}$ in Theorem 23 specifies to

$$
\mathbb{A}=\left(\alpha_{1}-v \alpha_{2}\right) L(\cdot, D) m^{-1}(m-\nu n)^{-1} .
$$

We observe that assumptions on $\mathbb{A}$ are satisfied if $\alpha_{1}(x)-$ $\nu \alpha_{2}(x) \geq c>0$ and $m(x)-\nu n(x) \geq c>0$ for all $x \in \bar{\Omega}$.

We compute now $\bar{z}$ :

$$
\begin{aligned}
\bar{z}(x)= & z_{1}(x)-\alpha_{2}(x)\left[\alpha_{2}(x)-\frac{m(x)}{n(x)} \alpha_{4}(x)\right]^{-1} \\
& \times\left[z_{1}(x)-\frac{m(x)}{n(x)} z_{2}(x)\right]+\left[\alpha_{1}(x)-\nu \alpha_{2}(x)\right] \\
& \times L(x, D)[m(x)-v n(x)]^{-1} n(x) L(x, D)^{-1} \\
& \times\left[\alpha_{2}(x)-\frac{m(x)}{n(x)} \alpha_{4}(x)\right]^{-1}\left[z_{1}(x)-\frac{m(x)}{n(x)} z_{2}(x)\right] .
\end{aligned}
$$

Finally, observe that in this case we have

$$
\begin{gathered}
S=m(m-v n) I \\
L_{1}-L_{2} L_{4}^{-1} L_{3}=\left(\alpha_{1}-v \alpha_{2}\right) L(\cdot, D), \\
L_{2} L_{4}^{-1}=\alpha_{2}\left(\alpha_{2}-\frac{m}{n} \alpha_{4}\right) I, \\
M_{2} L_{4}^{-1}=m n L(\cdot, D)^{-1}\left(\alpha_{2}-\frac{m}{n} \alpha_{4}\right) .
\end{gathered}
$$

Assume now that the data satisfy

$$
\begin{array}{r}
m^{2} u_{0}+m n v_{0} \in \mathscr{D}_{\mathbb{A}}(\theta+1, \infty), \quad \bar{z} \\
\in \mathscr{D}_{\mathbb{A}}\left(\theta_{0}, \infty\right), \\
0<\theta<\theta_{0}<1,
\end{array}
$$

$$
\begin{gathered}
\int_{\Omega} \eta(x) \bar{z}(x) d x \neq 0, \\
g \in C^{1+\theta}([0, \tau] ; \mathbb{R}), \\
g(0)=\int_{\Omega} \eta(x)\left[m(x)^{2} u_{0}(x)+m(x) n(x) v_{0}(x)\right] d x .
\end{gathered}
$$

Then Problem (237) admits a unique strict global solution $(u, v, f) \in C^{\theta}([0, \tau] ; \mathscr{D}(L)) \times C^{\theta}([0, \tau] ; \mathscr{D}(L)) \times C^{\theta}([0, \tau] ; \mathbb{R})$ such that $m^{2} u+m n v \in C^{\theta}\left([0, \tau] ; L^{p}(\Omega)\right), p \in(1,+\infty)$.

We now face the general case where the additional information is

$$
\begin{aligned}
\int_{\Omega} & \left\{\eta_{1}(x)\left[m(x)^{2} u(t, x)+m(x) n(x) v(t, x)\right]\right. \\
& \left.+\eta_{2}(x)\left[m(x) n(x) u(t, x)+n(x)^{2} v(t, x)\right]\right\} d x \\
= & g(t), \quad 0 \leq t \leq \tau .
\end{aligned}
$$

Taking advantage of the identity

$$
\begin{aligned}
m(x) & n(x) u(t, x)+n(x)^{2} v(t, x) \\
= & \frac{n(x)}{m(x)}\left[m(x)^{2} u(t, x)+m(x) n(x) v(t, x)\right],
\end{aligned}
$$


condition (246) takes the form

$$
\begin{aligned}
& \int_{\Omega}\left(\eta_{1}(x)+\frac{n(x)}{m(x)} \eta_{2}(x)\right) \\
& \quad \times\left[m(x)^{2} u(t, x)+m(x) n(x) v(t, x)\right] d x \\
&=g(t), \quad 0 \leq t \leq \tau .
\end{aligned}
$$

Moreover, solving (238) for $v$, we get the following equation for all $(t, x) \in[0, \tau] \times \Omega$ :

$$
\begin{aligned}
& v(t, x) \\
& =-v u(t, x)+f(t) L\left(x, D_{x}\right)^{-1} \\
& \quad \times\left(\alpha_{2}(x)-\frac{m(x)}{n(x)} \alpha_{4}(x)\right)^{-1}\left[z_{1}(x)-\frac{m(x)}{n(x)} z_{2}(x)\right],
\end{aligned}
$$

so that

$$
\begin{aligned}
& m(x)^{2} u(t, x)+m(x) n(x) v(t, x) \\
& =\left[m(x)^{2}-m(x) n(x)\right] u(t, x) \\
& +f(t) m(x) n(x) L\left(x, D_{x}\right)^{-1}\left(\alpha_{2}(x)-\frac{m(x)}{n(x)} \alpha_{4}(x)\right)^{-1} \\
& \quad \times\left[z_{1}(x)-\frac{m(x)}{n(x)} z_{2}(x)\right] \\
& \alpha_{1}(x) L\left(x, D_{x}\right) u(t, x)+\alpha_{2}(x) L\left(x, D_{x}\right) v(t, x) \\
& =\left[\alpha_{1}(x)-v \alpha_{2}(x)\right] L\left(x, D_{x}\right) u(t, x) \\
& \quad+f(t) \alpha_{2}(x)\left(\alpha_{2}(x)-\frac{m(x)}{n(x)} \alpha_{4}(x)\right)^{-1} \\
& \quad \times\left[z_{1}(x)-\frac{m(x)}{n(x)} z_{2}(x)\right] .
\end{aligned}
$$

Consequently, our identification problem is reduced to the previous one with $\eta$ being replaced by $\eta_{1}+n \eta_{2} / m$.

Problem 41 . Let $\Omega$ be a bounded region in $\mathbb{R}^{n}$ with a smooth boundary $\partial \Omega$. We are concerned with recovering function $f$ in the following problem related to a degenerate parabolic weakly coupled linear system:

$$
\begin{array}{r}
D_{t} u(t, x)=\Delta[a(x) u(t, x)]+b(x) v(t, x)+f(t) z_{1}(x), \\
(t, x) \in(0, \tau] \times \Omega, \\
D_{t} v(t, x)=c(x) u(t, x)+\Delta[d(x) v(t, x)]+f(t) z_{2}(x), \\
(t, x) \in(0, \tau] \times \Omega, \\
u(0, x)=u_{0}(x), \quad v(0, x)=v_{0}(x), \quad x \in \Omega, \\
a(x) u(t, x)=0, \quad d(x) v(t, x)=0, \\
\quad(t, x) \in(0, \tau] \times \partial \Omega, \\
\int_{\Omega}\left\{\eta_{1}(x) u(t, x)+\eta_{2}(x) v(t, x)\right\} d x=g(t), \quad t \in[0, \tau] .
\end{array}
$$

We assume that functions $a, b, c, d \in C(\bar{\Omega} ; \mathbb{R})$ and $a(x)>0$, $d(x)>0$ for a.e. $x \in \Omega$, while $z_{1}, z_{2}, \eta_{1}, \eta_{2} \in L^{2}(\Omega)=: X$, $u_{0}, v_{0} \in H_{0}^{1}(\Omega) \cap H^{2}(\Omega)$, and $g \in C([0, \tau] ; \mathbb{R})$. Finally, we assume that the consistency condition

$$
\int_{\Omega}\left\{\eta_{1}(x) u_{0}(x)+\eta_{2}(x) v_{0}(x)\right\} d x=g(0)
$$

is fulfilled.

Recall now that if $1 / a \in L^{r(n)}(\Omega)$, where

$$
r(1) \geq 2, \quad r(2)>2, \quad r(n) \geq n, \quad \text { if } n \geq 3,
$$

then [3, page 83$]$ operator $K$ defined by

$$
\begin{gathered}
\mathscr{D}(K)=\left\{u \in L^{2}(\Omega): a u \in H_{0}^{1}(\Omega) \cap H^{2}(\Omega)\right\}, \\
K u=-\Delta(a u), \\
u \in \mathscr{D}(K),
\end{gathered}
$$

satisfies the resolvent bound:

$$
\left\|(\lambda+K)^{-1}\right\|_{\mathscr{L}\left(L^{2}(\Omega)\right)} \leq c|\lambda|^{-(2 r-n) /(2 r)}
$$

for all $\lambda$ 's in a sector containing the closed half complex plane $\operatorname{Re} \lambda \geq 0$. This implies $\alpha=1$ and $\beta=(2 r-n) /(2 r)$.

Suppose now that $1 / a \in L^{r_{1}(n)}(\Omega)$ and $1 / d \in L^{r_{4}(n)}(\Omega)$, so that the corresponding differential operators $A$ and $D$ defined by

$$
\begin{gathered}
\mathscr{D}(A)=\left\{u \in L^{2}(\Omega): a u \in H_{0}^{1}(\Omega) \cap H^{2}(\Omega)\right\}, \\
A u=-\Delta(a u), \\
u \in \mathscr{D}(A), \\
\mathscr{D}(D)=\left\{v \in L^{2}(\Omega): d v \in H_{0}^{1}(\Omega) \cap H^{2}(\Omega)\right\}, \\
D v=-\Delta(d v), \\
v \in \mathscr{D}(D),
\end{gathered}
$$


satisfy $(\mathrm{H} 1)$ and $(\mathrm{H} 3)$ in Theorem 3 , with $B_{1}=B_{4}=I, \alpha=1$, $\beta_{1}=\left[2 r_{1}(n)-n\right] /\left[2 r_{1}(n)\right], \beta_{4}=\left[2 r_{4}(n)-n\right] /\left[2 r_{4}(n)\right]$.

Let $B$ and $C$ be the multiplication operators by $b(x)$ and $c(x)$ in $L^{2}(\Omega)$, respectively.

Theorem 3 runs provided

$$
\begin{gathered}
\min \left\{1-\frac{n}{2 r_{1}(n)}, 1-\frac{n}{2 r_{4}(n)}\right\}>\frac{1}{2} \\
\Longleftrightarrow \min \left\{r_{1}(n), r_{4}(n)\right\}>n,
\end{gathered}
$$

when $\theta_{0}=: n\left[2 \min \left\{r_{1}(n), r_{4}(n)\right\}\right]^{-1}$.

Whence it follows that if $\theta \in\left(\theta_{0}, 1-\theta_{0}\right), z_{1} \in \mathscr{D}(A)$, $z_{4} \in \mathscr{D}(D), \Delta\left(a u_{0}\right)+b v_{0} \in \mathscr{D}\left(L_{1}\right), \Delta\left(b v_{0}\right)+c u_{0} \in \mathscr{D}\left(L_{4}\right)$, $g \in C^{1+\theta}([0, \tau] ; \mathbb{R})$

$$
\begin{gathered}
\int_{\Omega}\left\{\eta_{1}(x) z_{1}(x)+\eta_{2}(x) z_{2}(x)\right\} d x \neq 0, \\
\int_{\Omega}\left\{\eta_{1}(x) u_{0}(x)+\eta_{2}(x) v_{0}(x)\right\} d x=g(0),
\end{gathered}
$$

then Problem (251) admits a unique strict global solution $(u, v, f) \in C^{\theta}\left([0, \tau] ; \mathscr{D}\left(L_{1}\right)\right) \times C^{\theta}\left([0, \tau] ; \mathscr{D}\left(L_{4}\right)\right) \times C^{\theta}([0, \tau] ; \mathbb{R})$ such that $u, v \in C^{1+\theta}\left([0, \tau] ; L^{2}(\Omega)\right)$.

More generally, consider the degenerate parabolic identification problem:

$$
\begin{aligned}
& D_{t} {[m(x) u(t, x)] } \\
&=\Delta[a(x) u(t, x)]+b(x) v(t, x)+f(t) z_{1}(x), \\
&(t, x) \in(0, \tau] \times \Omega,
\end{aligned}
$$

$$
\begin{aligned}
D_{t} & {[n(x) v(t, x)] } \\
=c(x) v(t, x)+\Delta[d(x) v(t, x)]+ & f(t) z_{2}(x), \\
& (t, x) \in(0, \tau] \times \Omega,
\end{aligned}
$$$$
\lim _{t \rightarrow 0+} m(x) u(t, x)=m(x) u_{0}(x),
$$$$
\lim _{t \rightarrow 0+} n(x) v(t, x)=n(x) v_{0}(x),
$$$$
x \in \Omega,
$$$$
a(x) u(t, x)=0, \quad d(x) v(t, x)=0,
$$$$
(t, x) \in(0, \tau] \times \partial \Omega,
$$$$
\int_{\Omega}\left\{\eta_{1}(x) m(x) u(t, x)+\eta_{2}(x) n(x) v(t, x)\right\} d x=g(t),
$$$$
t \in[0, \tau]
$$

where $m, n \in C(\bar{\Omega} ; \mathbb{R})$ and satisfy $m(x)>0, n(x)>0$ for all $x \in \Omega$.
The change of unknown functions $m u=u_{1}$ and $n v=v_{1}$ leads to the following (equivalent) identification problem:

$$
\begin{aligned}
& D_{t} u_{1}(t, x) \\
& =\Delta\left[\frac{a(x)}{m(x)} u_{1}(t, x)\right]+\frac{b(x)}{m(x)} v_{1}(t, x)+f(t) z_{1}(x), \\
& (t, x) \in(0, \tau] \times \Omega \\
& D_{t} v_{1}(t, x)=\frac{c(x)}{n(x)} u_{1}(t, x) \\
& +\Delta\left[\frac{d(x)}{n(x)} v_{1}(t, x)\right]+f(t) z_{2}(x), \\
& (t, x) \in(0, \tau] \times \Omega, \\
& u_{1}(0, x)=m(x) u_{0}(x), \\
& v_{1}(0, x)=n(x) v_{0}(x) \text {, } \\
& x \in \Omega \text {, } \\
& \frac{a(x)}{m(x)} u_{1}(t, x)=0, \quad \frac{d(x)}{n(x)} v_{1}(t, x)=0, \\
& (t, x) \in(0, \tau] \times \partial \Omega, \\
& \int_{\Omega}\left\{\eta_{1}(x) u_{1}(t, x)+\eta_{2}(x) v_{1}(t, x)\right\} d x=g(t), \\
& t \in[0, \tau] .
\end{aligned}
$$

Suppose that $a / m, d / n, b / m, c / n \in C(\bar{\Omega} ; \mathbb{R})$ - so that $b$ and $c$ must vanish on $\partial \Omega$ if $m$ and $n$ do. Moreover, we assume $m / a \in L^{r_{1}(n)}(\Omega) n / d \in L^{r_{4}(n)}(\Omega), r_{1}(n)$ and $r_{4}(n)$ being defined as above and satisfy $\min \left\{r_{1}(n), r_{4}(n)\right\}>n$, as well as

$$
\begin{gathered}
\mathscr{D}\left(\mathscr{L}_{1}\right)=\left\{u \in L^{2}(\Omega):\left(\frac{a}{m}\right) u \in H_{0}^{1}(\Omega) \cap H^{2}(\Omega)\right\}, \\
\mathscr{L}_{1} u=-\Delta\left(\left(\frac{a}{m}\right) u\right), \\
u \in \mathscr{D}\left(\mathscr{L}_{1}\right), \\
\mathscr{D}\left(\mathscr{L}_{4}\right)=\left\{v \in L^{2}(\Omega):\left(\frac{d}{n}\right) v \in H_{0}^{1}(\Omega) \cap H^{2}(\Omega)\right\}, \\
\mathscr{L}_{4} v=-\Delta\left(\left(\frac{d}{n}\right) v\right), \\
v\left(a u_{0}\right)+b v_{0} \in \mathscr{D}\left(\mathscr{L}_{1}\right), \quad \Delta\left(d v_{0}\right)+c u_{0} \in \mathscr{D}\left(\mathscr{L}_{4}\right), \\
g \in C^{1+\theta}([0, \tau] ; \mathbb{R}), \quad \theta \in\left(\theta_{0}, 1-\theta_{0}\right), \\
\int_{\Omega}\left\{\eta_{1}(x) z_{1}(x)+\eta_{2}(x) z_{2}(x)\right\} d x \neq 0, \\
\int_{\Omega}\left\{\eta_{1}(x) m(x) u_{0}(x)+\eta_{2}(x) n(x) v_{0}(x)\right\} d x=g(0) .
\end{gathered}
$$


Then Problem (259) admits a unique strict global solution $(u, v, f) \in C^{\theta}([0, \tau] ; \mathscr{D}(A)) \times C^{\theta}([0, \tau] ; \mathscr{D}(D)) \times C^{\theta}([0, \tau] ; \mathbb{R})$ such that $m u, n v \in C^{1+\theta}\left([0, \tau] ; L^{2}(\Omega)\right)$.

Problem 42. Consider the following one-dimensional parabolic identification problem of Sobolev type:

$$
\begin{aligned}
& D_{t}\left[u(t, x)+D_{x}^{2} u(t, x)\right]-D_{x}^{2} u(t, x)+a(x) D_{x}^{2} v(t, x) \\
& +b(x) D_{x} v(t, x)+c(x) v(t, x) \\
& =f(t) z_{1}(x), \quad(t, x) \in(0, \tau] \times \Omega, \\
& D_{t}\left[v(t, x)+D_{x}^{2} v(t, x)\right]-D_{x}^{2} v(t, x)+d v(t, x) \\
& =f(t) z_{2}(x), \quad(t, x) \in(0, \tau] \times \Omega, \\
& \left(1+D_{x}^{2}\right) u(0, x)=\left(1+D_{x}^{2}\right) u_{0}(x), \\
& \left(1+D_{x}^{2}\right) v(0, x)=\left(1+D_{x}^{2}\right) v_{0}(x), \\
& x \in \Omega \text {, } \\
& u(t, 0)=u(t, \pi)=0, \quad D_{x} v(t, 0)=D_{x} v(t, \pi)=0, \\
& t \in(0, \tau], \\
& \int_{\Omega}\left\{\eta_{1}(x)\left(1+D_{x}^{2}\right) u(t, x)+\eta_{2}(x)\left(1+D_{x}^{2}\right) v(t, x)\right\} d x \\
& =g(t), \quad t \in[0, \tau] .
\end{aligned}
$$

Here $a, b, c \in C([0, \pi] ; \mathbb{R})$ and $d$ is a positive constant.

Introduce now the linear operators $L_{i}, i=1,2,4$, and $M_{j}$, $j=1,2,3,4$, defined by

$$
\begin{array}{r}
\mathscr{D}\left(L_{1}\right)=H_{0}^{1}((0, \pi)) \cap H^{2}((0, \pi)), \quad \begin{array}{r}
L_{1} u=-D_{x}^{2} u, \\
u \in \mathscr{D}\left(L_{1}\right),
\end{array} \\
\mathscr{D}\left(L_{2}\right)=H^{2}((0, \pi)), \quad L_{2} v=a D_{x}^{2} v+b D_{x} v+c v, \\
v \in \mathscr{D}\left(L_{2}\right), \\
\mathscr{D}\left(L_{4}\right)=\left\{v \in H^{2}((0, \pi)): D_{x} v(0)=D_{x} v(\pi)=0\right\}, \\
L_{4} v=-D_{x}^{2} v+d v, \\
v \in \mathscr{D}\left(L_{4}\right), \\
\mathscr{D}\left(M_{1+3 j}\right)=\mathscr{D}\left(L_{1+3 j}\right), \quad M_{1+3 j} u=\left(1+D_{x}^{2}\right) u, \\
u \in \mathscr{D}\left(L_{1+3 j}\right), \quad j=0,1, \\
M_{2}=M_{3}=O .
\end{array}
$$

Note that the pairs $\left(M_{1}, L_{1}\right)$ and $\left(M_{4}, L_{4}\right)$ satisfy our spectral assumptions with $\alpha=\beta_{1}=\beta_{4}=1$ so that we can apply Theorem 14 with $\delta=1$ provided we make the following assumptions:

$$
\begin{gathered}
z_{j}=\left(1+D_{x}^{2}\right) z_{j}^{*}, \quad z_{j}^{*} \in \mathscr{D}\left(L_{1+3 j}\right), \quad j=0,1, \\
-D_{x}^{2} u_{0}=\left(1+D_{x}^{2}\right) u_{0}^{*}-a D_{x}^{2} v_{0}-b D_{x} v_{0}-c v_{0}, \\
u_{0}^{*} \in \mathscr{D}\left(L_{1}\right), \\
-D_{x}^{2} v_{0}+d v_{0}=\left(1+D_{x}^{2}\right) v_{0}^{*}, \quad v_{0}^{*} \in \mathscr{D}\left(L_{4}\right), \\
g \in C^{1+\theta}([0, \tau] ; \mathbb{R}), \quad \theta \in(0,1), \quad \eta_{1}, \eta_{2} \in L^{2}((0, \pi)), \\
\int_{\Omega}\left\{\eta_{1}(x) z_{1}(x)+\eta_{2}(x) z_{2}(x)\right\} d x \neq 0, \\
\int_{\Omega}\left\{\eta_{1}(x)\left(1+D_{x}^{2}\right) u_{0}(x)+\eta_{2}(x)\left(1+D_{x}^{2}\right) v_{0}(x)\right\} d x \\
=g(0) .
\end{gathered}
$$

Then Problems (262)-(265) admit a unique strict global solution $(u, v, f) \in C^{\theta}\left([0, \tau] ; \mathscr{D}\left(L_{1}\right)\right) \times C^{\theta}\left([0, \tau] ; \mathscr{D}\left(L_{4}\right)\right) \times$ $C^{\theta}([0, \tau] ; \mathbb{R})$ such that $\left(1+D_{x}^{2}\right) u,\left(1+D_{x}^{2}\right) v \in C^{1+\theta}([0$, $\left.\tau] ; L^{2}((0, \pi))\right)$.

Problem 43. We want to point out a different approach for solving the identification problem described in Example 28. For the sake of simplicity, we confine ourselves to the problem:

$$
\begin{gathered}
D_{t}\left[\begin{array}{cc}
M & M \\
I & I
\end{array}\right]\left[\begin{array}{l}
x(t) \\
y(t)
\end{array}\right] \\
+\left[\begin{array}{cc}
L_{1} & O \\
O & L_{2}
\end{array}\right]\left[\begin{array}{l}
x(t) \\
y(t)
\end{array}\right]=f(t)\left[\begin{array}{l}
z_{1} \\
z_{2}
\end{array}\right], \\
\forall t \in[0, \tau], \\
{\left[\begin{array}{cc}
M & M \\
I & I
\end{array}\right]\left[\begin{array}{l}
x \\
y
\end{array}\right](0)=\left[\begin{array}{cc}
M & M \\
I & I
\end{array}\right]\left[\begin{array}{l}
x_{0} \\
y_{0}
\end{array}\right],} \\
\Phi_{1}[M(x+y)(t)]+\Phi_{2}[(x+y)(t)]=g(t), \\
\forall t \in[0, \tau],
\end{gathered}
$$

with a compatibility relation $g(0)=\Phi_{1}\left[M\left(x_{0}+y_{0}\right)\right]+\Phi_{2}\left[x_{0}+\right.$ $\left.y_{0}\right]$.

Here $M$ is a bounded linear operator in $X, L_{j}: D\left(L_{j}\right) \subset$ $X \rightarrow X, j=1,2$ are two densely defined linear operators in $X$ such that $L_{2}, M+L_{1} L_{2}^{-1}$, and $z\left(M+L_{1} L_{2}^{-1}\right)+L_{1}$, Re $z \geq 0$, are invertible with inverses in $\mathscr{L}(X)$ and

$$
\left\|\left[z\left(M+L_{1} L_{2}^{-1}\right)+L_{1}\right]^{-1}\right\|_{\mathscr{L}(X)} \leq C(|z|+1)^{-1}, \quad \operatorname{Re} z \geq 0,
$$

$L_{1}$ being a sectorial operator with $\mathscr{D}\left(L_{2}\right) \subset \mathscr{D}\left(L_{1}\right)$. 
From the matrix equation in (265) we obtain the following equation for the new unknown $u=x+y$ :

$$
\begin{aligned}
f(t) z_{1} & =D_{t} M u(t)+L_{1} u(t)-L_{1} y(t) \\
& =D_{t} M u(t)+L_{1} u(t)-L_{1} L_{2}^{-1}\left[f(t) z_{2}-D_{t} u(t)\right] .
\end{aligned}
$$

Introduce now the linear functional defined by

$\Psi[w]=\Phi_{1}\left[M\left(M+L_{1} L_{2}^{-1}\right)^{-1} w\right]+\Phi_{2}\left[\left(M+L_{1} L_{2}^{-1}\right)^{-1} w\right]$

Then the pair $(u, f)$ solves the identification problem:

$$
\begin{gathered}
D_{t}\left[M+L_{1} L_{2}^{-1}\right] u(t)+L_{1} u(t) \\
=f(t)\left[z_{1}+L_{1} L_{2}^{-1} z_{2}\right], \quad t \in[0, \tau], \\
\left(M+L_{1} L_{2}^{-1}\right) u(0)=\left(M+L_{1} L_{2}^{-1}\right)\left(x_{0}+y_{0}\right), \\
\Psi\left[\left(M+L_{1} L_{2}^{-1}\right) u(t)\right]=g(t), \quad t \in[0, \tau] .
\end{gathered}
$$

Finally, set

$$
\xi(t)=\left[M+L_{1} L_{2}^{-1}\right] u(t) \Longleftrightarrow u(t)=\left[M+L_{1} L_{2}^{-1}\right]^{-1} \xi(t),
$$

It is immediate to check that function $\xi$ solves the nondegenerate identification problem:

$$
\begin{gathered}
D_{t} \xi(t)+L_{1}\left(M+L_{1} L_{2}^{-1}\right)^{-1} \xi(t)=f(t)\left[z_{1}+L_{1} L_{2}^{-1} z_{2}\right], \\
t \in[0, \tau], \\
\xi(0)=\left(M+L_{1} L_{2}^{-1}\right)\left(x_{0}+y_{0}\right), \\
\Psi[\xi(t)]=g(t), \quad t \in[0, \tau] .
\end{gathered}
$$

We can now apply [1, Theorem 2.1] if the following conditions are satisfied:

$$
\begin{gathered}
0<\theta<\theta_{0}<1, \quad g \in C^{1+\theta}([0, \tau] ; \mathbb{R}) ; \\
\sup _{t>0} t^{\theta}\left\|L_{1}\left[t\left(M+L_{1} L_{2}^{-1}\right)+L_{1}\right]^{-1} L_{1}\left(x_{0}+y_{0}\right)\right\|<+\infty \\
\Longleftrightarrow L_{1}\left(x_{0}+y_{0}\right) \in \mathscr{D}_{L_{1}\left(M+L_{1} L_{2}^{-1}\right)^{-1}}(\theta, \infty) ; \\
\sup _{t>0} t^{\theta_{0}}\left\|L_{1}\left[t\left(M+L_{1} L_{2}^{-1}\right)+L_{1}\right]^{-1}\left(z_{1}+L_{1} L_{2}^{-1} z_{2}\right)\right\|<+\infty \\
\Longleftrightarrow z_{1}+L_{1} L_{2}^{-1} z_{2} \in \mathscr{D}_{L_{1}\left(M+L_{1} L_{2}^{-1}\right)^{-1}}\left(\theta_{0}, \infty\right) ; \\
\Phi_{1}\left[M\left(x_{0}+y_{0}\right)\right]+\Phi_{2}\left[x_{0}+y_{0}\right]=g(0) ; \\
\Psi\left[z_{1}+L_{1} L_{2}^{-1} z_{2}\right] \neq 0 .
\end{gathered}
$$

Since

$$
\begin{aligned}
{[t I+} & \left.\left(M+L_{1} L_{2}^{-1}\right)^{-1} L_{1}\right]^{-1} \\
= & {\left[t\left(M+L_{1} L_{2}^{-1}\right)+L_{1}\right]^{-1}\left(M+L_{1} L_{2}^{-1}\right) } \\
= & \left(M+L_{1} L_{2}^{-1}\right)^{-1}\left[t I+L_{1}\left(M+L_{1} L_{2}^{-1}\right)\right]^{-1} \\
& \times\left(M+L_{1} L_{2}^{-1}\right)
\end{aligned}
$$

it follows that $\left(M+L_{1} L_{2}^{-1}\right)^{-1} L_{1}$ is sectorial, too. Moreover, from

$$
\begin{aligned}
t^{\theta} L_{1} & \left(M+L_{1} L_{2}^{-1}\right)^{-1}\left[t I+L_{1}\left(M+L_{1} L_{2}^{-1}\right)^{-1}\right]^{-1} \\
= & t^{\theta} L_{1}\left[t\left(M+L_{1} L_{2}^{-1}\right)+L_{1}\right]^{-1} \\
= & t^{\theta} L_{1}\left[t I+\left(M+L_{1} L_{2}^{-1}\right)^{-1} L_{1}\right]^{-1}\left(M+L_{1} L_{2}^{-1}\right)^{-1} \\
= & t^{\theta}\left(M+L_{1} L_{2}^{-1}\right)\left(M+L_{1} L_{2}^{-1}\right)^{-1} \\
& \times L_{1}\left[t I+\left(M+L_{1} L_{2}^{-1}\right)^{-1} L_{1}\right]^{-1}\left(M+L_{1} L_{2}^{-1}\right)^{-1},
\end{aligned}
$$

we deduce that $\xi \in \mathscr{D}_{L_{1}\left(M+L_{1} L_{2}^{-1}\right)^{-1}}(\theta, \infty)$ if and only if $(M+$ $\left.L_{1} L_{2}^{-1}\right)^{-1} \xi \in \mathscr{D}_{\left(M+L_{1} L_{2}^{-1}\right)^{-1} L_{1}}(\theta, \infty)$.

Since $-\left(M+L_{1} L_{2}^{-1}\right)^{-1} L_{1}$ is sectorial and has domain $\mathscr{D}\left(L_{1}\right)$, from [19] (cf. also [20]), it follows $\mathscr{D}_{\left(M+L_{1} L_{2}^{-1}\right)^{-1} L_{1}}(\theta$, $\infty)=\mathscr{D}_{L_{1}}(\theta, \infty)$. Therefore $\xi \in \mathscr{D}_{L_{1}\left(M+L_{1} L_{2}^{-1}\right)^{-1}}(\theta, \infty)$ if and only if $\left(M+L_{1} L_{2}^{-1}\right)^{-1} \xi \in \mathscr{D}_{L_{1}}(\theta, \infty)$.

Consequently, our condition on the data read equivalently

$$
\begin{gathered}
\left(M+L_{1} L_{2}^{-1}\right)^{-1} L_{1}\left(x_{0}+y_{0}\right) \in \mathscr{D}_{L_{1}}(\theta, \infty), \\
\left(M+L_{1} L_{2}^{-1}\right)^{-1}\left(z_{1}+L_{1} L_{2}^{-1} z_{2}\right) \in \mathscr{D}_{L_{1}}\left(\theta_{0}, \infty\right) .
\end{gathered}
$$

Then Problem (265) admits a unique solution $(\xi, f) \in$ $\left[C^{1+\theta}([0, \tau] ; X) \cap C^{\theta}\left([0, \tau] ; \mathscr{D}\left(L_{1}\left(M+L_{1} L_{2}^{-1}\right)^{-1}\right)\right)\right] \times C^{\theta}([0$, $\tau] ; \mathbb{R})$. Consequently, since $u(t)=\left[M+L_{1} L_{2}^{-1}\right]^{-1} \xi(t)$ and $\xi \in C^{1+\theta}([0, \tau] ; X) \cap C^{\theta}\left([0, \tau] ; \mathscr{D}\left(\left[M+L_{1} L_{2}^{-1}\right]^{-1}\right)\right)$, then the solution $(u, f)$ to Problem (269) has the properties $(M+$ $\left.L_{1} L_{2}^{-1}\right)^{-1} u \in C^{1+\theta}([0, \tau] ; X),(u, f) \in C^{\theta}\left([0, \tau] ; \mathscr{D}\left(L_{1}\right)\right) \times$ $C^{\theta}([0, \tau] ; \mathbb{R})$.

Finally, from the latter differential equation in (265) we easily deduce the following representation formula for the pair $(x, y)$ :

$$
\begin{gathered}
y(t)=-L_{2}^{-1} u(t)+f(t) L_{2}^{-1} z_{2}, \\
x(t)=u(t)-y(t)=u(t)+L_{2}^{-1} u(t)-f(t) L_{2}^{-1} z_{2}, \\
t \in[0, \tau] .
\end{gathered}
$$

We can conclude by stating that Problem (269) admits a unique solution $(x, y, f) \in C^{\theta}\left([0, \tau] ; \mathscr{D}\left(L_{1}\right)\right) \times C^{\theta}([0, \tau]$; $\left.\mathscr{D}\left(L_{2}\right)\right) \times C^{\theta}([0, \tau] ; \mathbb{R})$ such that $x+y \in C^{1+\theta}([0, \tau] ; X)$. 
Remark 44. If $f$ is not differentiable with respect to time, functions $x$ and $y$ need not to be differentiable, even though their sum is. This fact exhibits the degeneracy of our Problem (265).

Remark 45. Consider the identification problem:

$$
\begin{gathered}
D_{t}\left[\begin{array}{cc}
-I & -I \\
I & I
\end{array}\right]\left[\begin{array}{l}
x(t) \\
y(t)
\end{array}\right]+\left[\begin{array}{ll}
L & O \\
O & L
\end{array}\right]\left[\begin{array}{l}
x(t) \\
y(t)
\end{array}\right]=f(t)\left[\begin{array}{l}
z_{1} \\
z_{2}
\end{array}\right], \\
\forall t \in[0, \tau] \\
{\left[\begin{array}{cc}
-I & -I \\
I & I
\end{array}\right]\left[\begin{array}{l}
x \\
y
\end{array}\right](0)=\left[\begin{array}{cc}
-I & -I \\
I & I
\end{array}\right]\left[\begin{array}{l}
x_{0} \\
y_{0}
\end{array}\right]} \\
-\Phi_{1}[(x+y)(t)]+\Phi_{2}[(x+y)(t)]=g(t) \\
\forall t \in[0, \tau]
\end{gathered}
$$

with a compatibility relation $g(0)=\left(\Phi_{1}-\Phi_{2}\right)\left[x_{0}+y_{0}\right]$.

Here $L: D(L) \subset X \rightarrow X$ is a densely defined invertible linear operator in $X$ such that $L^{-1} \in \mathscr{L}(X)$, while $x_{0}, y_{0}$, $z_{1}, z_{2} \in X$ and $g \in C^{1}([0, \tau], \mathbb{R})$.

First we note that our assumptions are not satisfied, since $z(M+I)+L=L$ for all $z \in \mathbb{C}$. However, a trivial computation yields

$$
(x+y)(t)=f(t) L^{-1}\left(z_{1}+z_{2}\right), \quad \forall t \in[0, \tau] .
$$

Therefore, if

$$
\Phi_{1}\left[L^{-1}\left(z_{1}+z_{2}\right)\right] \neq \Phi_{2}\left[L^{-1}\left(z_{1}+z_{2}\right)\right]
$$

$f$ is uniquely determined by

$$
f(t)=\left(\Phi_{2}-\Phi_{1}\right)\left[L^{-1}\left(z_{1}+z_{2}\right)\right]^{-1} g(t), \quad \forall t \in[0, \tau] .
$$

Since

$$
\begin{array}{ll}
L x(t)=f(t) z_{1}+f^{\prime}(t) L^{-1}\left(z_{1}+z_{2}\right), & \forall t \in[0, \tau], \\
L y(t)=f(t) z_{2}-f^{\prime}(t) L^{-1}\left(z_{1}+z_{2}\right), & \forall t \in[0, \tau],
\end{array}
$$

then the assumptions on our data imply

$$
x+y \in C^{1}([0, \tau], X), \quad f \in C^{1}([0, \tau], \mathbb{R}),
$$

without any resolvent estimate involving $L$, but $0 \in \rho(L)$.

Application 1. Let $X=L^{p}(\Omega), p \in(1,+\infty), \Omega$ being a domain in $\mathbb{R}^{n}$ with a boundary $\partial \Omega$ of class $C^{2}$. Let $a_{i, j} \in C(\bar{\Omega} ; \mathbb{R})$ with $a_{i, j}=a_{j, i}, i, j=1, \ldots, n$, be given function satisfying the ellipticity condition:

$$
\sum_{i, j=1}^{n} a_{i, j}(x) \xi_{i} \xi_{j} \geq c_{0}|\xi|^{2}, \quad(x, \xi) \in \bar{\Omega} \times \mathbb{R}^{n} .
$$

Define then the linear differential second-order operator by

$$
\begin{aligned}
\mathscr{D}(L) & =W^{2, p}(\Omega) \cap W_{0}^{1, p}(\Omega), \\
L u & =\sum_{i, j=1}^{n} a_{i, j} D_{x_{i}} D_{x_{j}}-a_{0} u,
\end{aligned}
$$

where $a_{0} \in C\left(\bar{\Omega} ; \mathbb{R}_{+}\right)$.

Introduce also two functions $m, a_{1} \in C(\bar{\Omega} ; \mathbb{R})$ such that $a_{1}(x)>0$ and $m(x)+a_{1}(x)^{-1} \geq c_{1}>0$ for all $x \in \bar{\Omega}$ and denote by $M$ the multiplication operator in $L^{p}(\Omega)$ corresponding to $m$.

Following the same steps as in [3, pages 79-80], we can readily derive the resolvent estimate

$$
\left\|\left[z\left(M+a_{1}^{-1} I\right)-L\right]^{-1}\right\|_{\mathscr{L}(X)} \leq C(1+|z|)^{-1}, \quad \text { if } \operatorname{Re} z \geq 0 .
$$

Consider then the identification problem:

$$
\begin{gathered}
D_{t}\left[\begin{array}{cc}
m(x) & m(x) \\
1 & 1
\end{array}\right]\left[\begin{array}{l}
u(t, x) \\
v(t, x)
\end{array}\right]-\left[\begin{array}{cc}
L & O \\
O & a_{1} L
\end{array}\right]\left[\begin{array}{l}
u(t, x) \\
v(t, x)
\end{array}\right] \\
=f(t)\left[\begin{array}{l}
z_{1}(x) \\
z_{2}(x)
\end{array}\right], \quad \forall t \in[0, \tau] \\
{\left[\begin{array}{cc}
m(x) & m(x) \\
1 & 1
\end{array}\right]\left[\begin{array}{l}
u(0, x) \\
v(0, x)
\end{array}\right]=\left[\begin{array}{cc}
m(x) & m(x) \\
1 & 1
\end{array}\right]\left[\begin{array}{l}
u_{0}(x) \\
v_{0}(x)
\end{array}\right],} \\
\int_{\Omega}\left[\eta_{1}(x) m(x)+\eta_{2}(x)\right][u(t, x)+v(t, x)] d x=g(t), \\
\forall t \in[0, \tau] .
\end{gathered}
$$

We assume that our data satisfy

$$
\begin{gathered}
u_{0}, v_{0} \in L^{p}(\Omega), \\
u_{0}+v_{0} \in W^{2, p}(\Omega) \cap W_{0}^{1, p}(\Omega), \\
\left(m+a_{1}^{-1}\right)^{-1} L\left(u_{0}+v_{0}\right) \in \mathscr{D}_{L}(\theta, \infty), \\
\left(m+a_{1}^{-1}\right)^{-1}\left(z_{1}+a_{1}^{-1} z_{2}\right) \in \mathscr{D}_{L}\left(\theta_{0}, \infty\right), \\
\eta_{1}+\eta_{2} \in L^{p^{\prime}}(\Omega), \quad \frac{1}{p}+\frac{1}{p^{\prime}}=1, \\
g \in C^{1+\theta}([0, \tau] ; \mathbb{R}), \\
g(0)=\int_{\Omega}\left[\eta_{1}(x) m(x)+\eta_{2}(x)\right]\left[u_{0}(x)+v_{0}(x)\right] d x, \\
\int_{\Omega}\left[\eta_{1}(x) m(x)+\eta_{2}(x)\right]\left[m(x)+a_{1}(x)^{-1}\right]^{-1} \\
\times\left[z_{1}(x)+a_{1}(x)^{-1} z_{2}(x)\right] d x \neq 0 .
\end{gathered}
$$

We note that the spaces $\mathscr{D}_{L}(\theta, \infty)$ are well characterised in [9, page 321]. 
By virtue of the previous result we deduce that our degenerate parabolic identification problem admits a unique global strict solution $(u, v, f) \in C^{\theta}([0, \tau] ; \mathscr{D}(L)) \times C^{\theta}([0, \tau] ; \mathscr{D}(L)) \times$ $C^{\theta}([0, \tau] ; \mathbb{R})$ such that $u+v \in C^{1+\theta}\left([0, \tau] ; L^{p}(\Omega)\right)$ and $m(u+$ $v) \in C^{1+\theta}\left([0, \tau] ; L^{p}(\Omega)\right)$.

Application 2. According to [21] the projection operator $P$ onto $N(T)$ relative to the direct sum representation $N(T) \oplus$ $\overline{R(T)}$ is well characterised. Now we show how the corresponding projection in product space $X \times X$ can be characterised as well.

For the sake of simplicity we confine ourselves to a bit less general case than the one discussed in Proposition 16, choosing here $M_{2}=O$. Hence the corresponding differential system becomes

$$
D_{t}\left[\begin{array}{cc}
M_{1} & O \\
O & M_{4}
\end{array}\right]\left[\begin{array}{l}
u(t) \\
v(t)
\end{array}\right]+\left[\begin{array}{cc}
L_{1} & L_{2} \\
O & L_{4}
\end{array}\right]\left[\begin{array}{l}
u(t) \\
v(t)
\end{array}\right]=f(t)\left[\begin{array}{l}
z_{1} \\
z_{2}
\end{array}\right],
$$$$
\forall t \in[0, \tau]
$$

Suppose that $L_{1}$ and $L_{4}$ admit bounded inverses satisfying

$$
\begin{aligned}
& \mathscr{D}\left(L_{2}\right) \subset \mathscr{D}\left(L_{4}\right), \quad \mathscr{D}\left(L_{j}\right) \subset \mathscr{D}\left(M_{j}\right), \quad j=1,4, \\
& \left\|z M_{i}\left(z M_{i}+L_{i}\right)^{-1}\right\|_{\mathscr{D}(X)} \leq c, \quad \text { if } \operatorname{Re} z \geq 0, \quad j=1,4 .
\end{aligned}
$$

From our assumption we deduce the following formula:

$$
\left[\begin{array}{cc}
L_{1} & L_{2} \\
O & L_{4}
\end{array}\right]^{-1}=\left[\begin{array}{cc}
L_{1}^{-1} & -L_{1}^{-1} L_{2} L_{4}^{-1} \\
O & L_{4}^{-1}
\end{array}\right]
$$

Setting $T_{i}=M_{i} L_{i}^{-1}, i=1$, 4 , we get

$$
\mathbb{T}=\left[\begin{array}{cc}
M_{1} & O \\
O & M_{4}
\end{array}\right]\left[\begin{array}{cc}
L_{1}^{-1} & -L_{1}^{-1} L_{2} L_{4}^{-1} \\
O & L_{4}^{-1}
\end{array}\right]=\left[\begin{array}{cc}
T_{1} & -T_{1} L_{2} L_{4}^{-1} \\
O & T_{4}
\end{array}\right] .
$$

(288)

Hence

$$
(z \mathbb{T}+I)^{-1}=\left[\begin{array}{cc}
\left(z T_{1}+I\right)^{-1} & L_{2} L_{4}^{-1}\left(z T_{4}+I\right)^{-1}-\left(z T_{1}+I\right)^{-1} L_{2} L_{4}^{-1}\left(z T_{4}+I\right)^{-1} \\
O & \left(z T_{4}+I\right)^{-1}
\end{array}\right] .
$$

Whence it follows

$$
\left\|(z \mathbb{T}+\llbracket)^{-1}\right\|_{\mathscr{L}(X \times X)} \leq C, \quad \text { if } \operatorname{Re} z \geq 0 .
$$

Note now that if $X$ is a reflexive Banach space, we have the decomposition:

$$
X \times X=N(\mathbb{T}) \oplus \overline{R(\mathbb{T})} .
$$

Observe then that

$$
(x, y) \in N(\mathbb{T}) \Longleftrightarrow T_{4} y=0, \quad T_{1}\left(x-L_{2} L_{4}^{-1} y\right)=0 .
$$

Introduce finally the linear bounded operator:

$$
\mathbb{P}=\left[\begin{array}{cc}
P_{1} & -P_{1} L_{2} L_{4}^{-1}+L_{2} L_{4}^{-1} P_{2} \\
O & P_{2}
\end{array}\right],
$$

where $P_{1}$ and $P_{2}$ are the projections on $N\left(T_{1}\right)$ and $N\left(T_{4}\right)$, respectively.

It is easily checked that $\mathbb{P}^{2}=\mathbb{P}$ and

$$
\mathbb{P}\left[\begin{array}{l}
x \\
y
\end{array}\right]=\left[\begin{array}{c}
P_{1} x-P_{1} L_{2} L_{4}^{-1} y+L_{2} L_{4}^{-1} P_{2} y \\
P_{2} y
\end{array}\right] \in N(\mathbb{T}),
$$

since $T_{4} P_{2} y=0$ and

$$
\begin{aligned}
& T_{1}\left(P_{1} x-P_{1} L_{2} L_{4}^{-1} y+L_{2} L_{4}^{-1} P_{2} y\right)-T_{1} L_{2} L_{4}^{-1} P_{2} y \\
& \quad=T_{1} L_{2} L_{4}^{-1} P_{2} y-T_{1} L_{2} L_{4}^{-1} P_{2} y=0 .
\end{aligned}
$$

Once we have characterised $\mathbb{P}$, we obtain the basic formula $\overline{R(\mathbb{T})}=(\square-\mathbb{P})(X \times X)$. Consequently, we have at our disposal all the elements required in Proposition 16. lem:

As an application, we can handle the identification prob-

$$
\begin{aligned}
& D_{t}\left[\begin{array}{cc}
m_{1}(x) & 0 \\
0 & m_{4}(x)
\end{array}\right]\left[\begin{array}{l}
u(t, x) \\
v(t, x)
\end{array}\right] \\
& \quad-\left[\begin{array}{cc}
L_{1}(x, D) & L_{2}(x, D) \\
O & L_{4}(x, D)
\end{array}\right]\left[\begin{array}{l}
u(t, x) \\
v(t, x)
\end{array}\right] \\
& =f(t)\left[\begin{array}{l}
z_{1}(x) \\
z_{2}(x)
\end{array}\right], \quad \forall t \in[0, \tau], \\
& \quad\left[\begin{array}{cc}
m_{1}(x) & 0 \\
0 & m_{4}(x)
\end{array}\right]\left[\begin{array}{l}
u(0, x) \\
v(0, x)
\end{array}\right] \\
& =\left[\begin{array}{cc}
m_{1}(x) & 0 \\
0 & m_{4}(x)
\end{array}\right]\left[\begin{array}{l}
u_{0}(x) \\
v_{0}(x)
\end{array}\right], \\
& \left\langle m_{1} u, \eta_{1}\right\rangle_{H^{-1}(\Omega) \times H_{0}^{1}(\Omega)} \\
& \quad+\left\langle m_{2} v, \eta_{2}\right\rangle_{H^{-1}(\Omega) \times H_{0}^{1}(\Omega)}=g(t), \quad \forall t \in[0, \tau] .
\end{aligned}
$$

Assume now that $m_{1}, m_{2}$ are nonnegative functions in $L^{\infty}(\Omega), z_{1}, z_{2} \in H^{-1}(\Omega), u_{0}, v_{0}, \eta_{1}, \eta_{2} \in H_{0}^{1}(\Omega)$, while $L_{1}(x$, $D), L_{4}(x, D)$, and $L_{2}(x, D)$ are second-order linear operators such that the first two are isomorphisms from $H_{0}^{1}(\Omega)$ into $H^{-1}(\Omega)$, while the third one is bounded from $H_{0}^{1}(\Omega)$ into $H^{-1}(\Omega)$. 
Under such conditions, the conditions in Proposition 16 ensuring the solvability of the identification Problem (299) are fulfilled. Such conditions, related to the projection $\mathbb{P}$, are expressed by means of the projections $P_{1}$ and $P_{2}$.

Application 3. We generalize here to a system Problem 9 related to the scalar Ornstein-Uhlenbeck equation and use the same notation.

Let $\widetilde{m}$ be a uniformly continuous and bounded function on $\mathbb{R}^{N}$ such that

$$
\widetilde{m}(x) \geq c_{0}>0, \quad x \in \mathbb{R}^{N} .
$$

Consider first, for all $\lambda \in \mathbb{C}_{+}=\{z \in \mathbb{C}: \operatorname{Re} z \geq 0\}$ and $f \in L_{\mu}^{2}\left(\mathbb{R}^{N}\right)$, the resolvent equation:

$$
\lambda \widetilde{m} u+\left(-\mathscr{L}_{2}+I\right) u=f
$$

where

$$
B Q=Q B^{*}, \quad B=-Q_{\infty}^{-1} .
$$

Then $-\mathscr{L}_{2}$ is self-adjoint in $L_{\mu}^{2}\left(\mathbb{R}^{N}\right)$ and [19] (cf. [20, Section 9.3.2, page 251]):

$$
\int_{\mathbb{R}^{n}} g \cdot \mathscr{L}_{2} f d \mu=-\int_{\mathbb{R}^{N}}\langle Q D f, D g\rangle d \mu .
$$

Thus, taking the real and imaginary parts in the equality:

$$
\begin{gathered}
\lambda \int_{\mathbb{R}^{N}} \widetilde{m}|u|^{2} d \mu+\int_{\mathbb{R}^{N}}\langle Q D u, D \bar{u}\rangle d \mu \\
+\int_{\mathbb{R}^{N}}|u|^{2} d \mu=\int_{\mathbb{R}^{N}} f \bar{u} d \mu,
\end{gathered}
$$

we get the relations as follows:

$$
\begin{aligned}
& \operatorname{Re} \lambda \int_{\mathbb{R}^{N}} \widetilde{m}|u|^{2} d \mu+\int_{\mathbb{R}^{N}}\langle Q D u, D \bar{u}\rangle d \mu \\
& \quad+\int_{\mathbb{R}^{N}}|u|^{2} d \mu=\operatorname{Re} \int_{\mathbb{R}^{N}} f \bar{u} d \mu, \\
& \operatorname{Im} \lambda \int_{\mathbb{R}^{N}} \widetilde{m}|u|^{2} d \mu=\operatorname{Im} \int_{\mathbb{R}^{N}} f \bar{u} d \mu .
\end{aligned}
$$

They, in turn, imply

$$
\begin{aligned}
& {[\operatorname{Re} \lambda+|\operatorname{Im} \lambda|] \int_{\mathbb{R}^{N}} \widetilde{m}|u|^{2} d \mu} \\
& \quad+\int_{\mathbb{R}^{N}}\langle Q D u, D \bar{u}\rangle d \mu+\int_{\mathbb{R}^{N}}|u|^{2} d \mu \\
& \leq 2\|f\|_{L_{\mu}^{2}\left(\mathbb{R}^{N}\right)}\|u\|_{L_{\mu}^{2}\left(\mathbb{R}^{N}\right)} .
\end{aligned}
$$

Since $\operatorname{Re} \lambda+|\operatorname{Im} \lambda| \geq 0$, we deduce

$$
\int_{\mathbb{R}^{N}}\langle Q D u, D \bar{u}\rangle d \mu+\int_{\mathbb{R}^{N}}|u|^{2} d \mu \leq 2\|f\|_{L_{\mu}^{2}\left(\mathbb{R}^{N}\right)}\|u\|_{L_{\mu}^{2}\left(\mathbb{R}^{N}\right)} .
$$

implying

$$
\begin{aligned}
c_{0}|\lambda| \int_{\mathbb{R}^{N}}|u|^{2} d \mu & \leq|\lambda| \int_{\mathbb{R}^{N}} \widetilde{m}|u|^{2} d \mu \\
& \leq[\operatorname{Re} \lambda+|\operatorname{Im} \lambda|] \int_{\mathbb{R}^{N}} \widetilde{m}|u|^{2} d \mu \\
& \leq 4\|f\|_{L_{\mu}^{2}\left(\mathbb{R}^{N}\right)} \\
& =4\left\|\lambda \widetilde{m} u+\left(-\mathscr{L}_{2}+I\right) u\right\|_{L_{\mu}^{2}\left(\mathbb{R}^{N}\right)}^{2}, \quad \lambda \in \mathbb{C}_{+} .
\end{aligned}
$$

Since $\left[\lambda \widetilde{m} I+\left(-\mathscr{L}_{2}+I\right)\right]^{*}=\bar{\lambda} \widetilde{m} I+\left(-\mathscr{L}_{2}+I\right)$, we can conclude that $\lambda \widetilde{m} I+\left(-\mathscr{L}_{2}+I\right)$ has a bounded inverse and

$$
\left\|\left[\lambda \widetilde{m} I+\left(-\mathscr{L}_{2}+I\right)\right]^{-1}\right\|_{\mathscr{L}\left(L_{\mu}^{2}\left(\mathbb{R}^{N}\right)\right)} \leq 4 c_{0}^{-1}|\lambda|^{-1}, \quad \lambda \in \mathbb{C}_{+} .
$$

Let us now consider the following degenerate identification problem related to a matrix-valued Ornstein-Uhlenbeck operator: look for a triplet of functions $u, v: \mathbb{R}^{N} \rightarrow \mathbb{R}$ and $f:[0, T] \rightarrow \mathbb{R}$ such that

$$
\begin{aligned}
& D_{t}\left[\begin{array}{cc}
m(x) & m(x) \\
I & I
\end{array}\right]\left[\begin{array}{l}
u(t, x) \\
v(t, x)
\end{array}\right] \\
& \quad-\left[\begin{array}{cc}
\mathscr{L}_{2}-I & O \\
O & a_{1}(x)\left(\mathscr{L}_{2}-I\right)
\end{array}\right]\left[\begin{array}{l}
u(t, x) \\
v(t, x)
\end{array}\right] \\
& =f(t)\left[\begin{array}{l}
z_{1}(x) \\
z_{2}(x)
\end{array}\right], \quad \forall t \in[0, \tau], \quad x \in \mathbb{R}^{N}, \\
& {\left[\begin{array}{cc}
m(x) & m(x) \\
I & I
\end{array}\right]\left[\begin{array}{l}
u(0, x) \\
v(0, x)
\end{array}\right]} \\
& =\left[\begin{array}{cc}
m(x) & m(x) \\
I & I
\end{array}\right]\left[\begin{array}{l}
u_{0}(x) \\
v_{0}(x)
\end{array}\right], \quad x \in \mathbb{R}^{N}, \\
& \int_{\mathbb{R}^{N}}\left[\begin{array}{c}
\left.\eta_{1}(x) m(x)+\eta_{2}\right][u(t, x)+v(t, x)] d \mu, \\
=
\end{array}=g(t), \quad \forall t \in[0, \tau],\right.
\end{aligned}
$$

where

$$
u_{0}, v_{0}, \eta_{1}, \eta_{2} \in L_{\mu}^{2}\left(\mathbb{R}^{N}\right)
$$

We now assume that functions $m$ and $a_{1}$ are uniformly continuous, bounded, and strictly positive in $\mathbb{R}^{N}$. Moreover, we assume that function $m+\left(1 / a_{1}\right)$ has the same properties and satisfies $m(x)+\left(1 / a_{1}(x)\right) \geq c_{0}>0$ for all $x \in \mathbb{R}^{N}$. Then condition (309) is satisfied with $\widetilde{m}=m+\left(1 / a_{1}\right)$. Further, the interpolation space is characterised by

$$
\left(L_{\mu}^{2}\left(\mathbb{R}^{N}\right), W_{\mu}^{2,2}\left(\mathbb{R}^{N}\right)\right)_{\theta, \infty}=B_{2, \infty}^{2 \theta}\left(\mathbb{R}^{N} ; \mu\right), \quad \theta \in(0,1) .
$$


Assume now that our data enjoy the following properties:

$$
\begin{gathered}
u_{0}, v_{0} \in L_{\mu}^{2}\left(\mathbb{R}^{N}\right), \quad u_{0}+v_{0} \in W_{\mu}^{2,2}\left(\mathbb{R}^{N}\right), \\
z_{1}, z_{2} \in L_{\mu}^{2}\left(\mathbb{R}^{N}\right), \quad \frac{\left(a_{1} z_{1}+z_{2}\right)}{\left(m a_{1}+1\right)} \in B_{2, \infty}^{2 \theta_{0}}\left(\mathbb{R}^{N} ; \mu\right), \\
g(0)=\int_{\mathbb{R}^{N}}\left[\eta_{1}(x) m(x)+\eta_{2}(x)\right]\left[\mathrm{u}_{2}\right), \\
\int_{\mathbb{R}^{N}}\left[\eta_{1}(x) m(x)+\eta_{0}(x)+v_{0}(x)\right] d \mu . \\
\times\left[\frac{1}{m(x) a_{1}(x)+1}\right]\left[a_{1} z_{1}(x)+z_{2}(x)\right] d x \neq 0 .
\end{gathered}
$$

Consequently, our degenerate parabolic identification problem possesses a unique global strict solution $(u, v, f) \in$ $C^{\theta}\left([0, \tau] ; W_{\mu}^{2,2}\left(\mathbb{R}^{N}\right)\right) \times C^{\theta}\left([0, \tau] ; W_{\mu}^{2,2}\left(\mathbb{R}^{N}\right)\right) \times C^{\theta}([0, \tau] ; \mathbb{R})$ such that $u+v, m(u+v) \in C^{1+\theta}\left([0, \tau] ; L_{\mu}^{2}\left(\mathbb{R}^{N}\right)\right)$.

Application 4. We are here concerned with hyperbolic systems.

Let $\mathscr{A}^{j}=\left(a_{h, k}^{j}\right)_{h, k=1}^{N}, j=1, \ldots, n, N, n \in \mathbb{R} \backslash\{0\}$, be a $N \times N$ Hermitian matrix, that is, $a_{h, k}^{j}=a_{k, h}^{j}$, and let $a_{h, k}^{j} \in$ $C_{b}^{1}\left(\mathbb{R}^{n} ; \mathbb{C}\right)$, the space of all bounded functions along with all first-order derivatives. Let $\mathscr{B}=\left(b_{h, k}\right)_{h, k=1}^{N}$ be a $N \times N$ matrix with $b_{h, k} \in C_{b}^{1}\left(\mathbb{R}^{n} ; \mathbb{C}\right)$.

Set $X=L^{2}\left(\mathbb{R}^{n}\right)^{N}$ and for all $u, v \in L^{2}\left(\mathbb{R}^{n}\right)^{N}$ define the inner product:

$$
\langle\langle u, v\rangle\rangle=\sum_{j=1}^{N}\left\langle u_{j}, v_{j}\right\rangle_{L^{2}\left(\mathbb{R}^{n}\right)}=\sum_{j=1}^{N} \int_{\mathbb{R}^{n}} u_{j}(x) \bar{v}_{j}(x) d x
$$

where $u=\left(u_{1}, \ldots, u_{N}\right)$ and $v=\left(v_{1}, \ldots, v_{N}\right)$, so that $\|u\|^{2}=$ $\sum_{j=1}^{N}\left\|u_{j}\right\|_{L^{2}\left(\mathbb{R}^{n}\right)^{N}}^{2}$.

If $T \in \mathcal{S}^{\prime}\left(\mathbb{R}^{n}\right), D_{x_{j}} T$ is the temperate distribution defined by $\left\langle D_{x_{j}} T, \varphi\right\rangle=-\left\langle T, D_{x_{j}} \varphi\right\rangle$ for all $\varphi \in \mathcal{S}\left(\mathbb{R}^{n}\right)$. If $f \in L^{2}\left(\mathbb{R}^{n}\right)$ and $a \in C^{1}\left(\mathbb{R}^{n} ; \mathbb{C}\right)$ one defines

$$
a D_{x_{j}} f=D_{x_{j}}(a f)-f D_{x_{j}} a .
$$

Of course, $f \in L^{2}\left(\mathbb{R}^{n}\right)$ is identified with the temperate distribution $\langle f, \varphi\rangle=\int_{\mathbb{R}^{n}} f(x) \varphi(x) d x, \varphi \in \mathcal{S}\left(\mathbb{R}^{n}\right)$.
Therefore the components of $\sum_{j=1}^{n} \mathscr{A}^{j}(x) D_{x_{j}} u+\mathscr{B}(x) u$ are given by

$$
\sum_{j=1}^{n} \sum_{l=1}^{N} a_{k, l}^{j}(x) D_{x_{j}} u_{l}+\sum_{l=1}^{N} b_{k, l}(x) u_{l}(x), \quad k=1, \ldots, N
$$

where $D_{x_{j}} u_{l}$ is meant in the sense of distributions.

We can now introduce the linear first-order differential operator $\mathscr{A}$ defined by

$$
\begin{aligned}
& \mathscr{A} u=\sum_{j=1}^{n} \mathscr{A}^{j}(x) D_{x_{j}} u+\mathscr{B}(x) u \in \mathcal{S}^{\prime}\left(\mathbb{R}^{n}\right), \quad u \in X, \\
& \mathscr{D}(A)=\{u \in X: \mathscr{A} u \in X\}, \quad A u=\mathscr{A} u, u \in \mathscr{D}(A) .
\end{aligned}
$$

It is well known (cf. [22]) that $A$ generates a $C_{0}$-semigroup in $X$. Consequently, our abstract identification problem reduces to the following: find a pair $(u, f)$ such that

$$
\begin{gathered}
D_{t} u_{k}=\sum_{j=1}^{n} \sum_{l=1}^{N} a_{k, l}^{j}(x) D_{x_{j}} u_{l}+\sum_{l=1}^{N} b_{k, l}(x) u_{l}(x)+f(t) z_{k} \\
(t, x) \in[0, \tau] \times \mathbb{R}^{n}, \quad k=1, \ldots, N \\
u_{k}(0, x)=u_{0, k}(x), \quad x \in \mathbb{R}^{n}, k=1, \ldots, N \\
\langle\langle\eta, u(t, \cdot)\rangle\rangle=g(t), \quad t \in[0, \tau]
\end{gathered}
$$

where $z=\left(z_{1}, \ldots, z_{N}\right), u_{0}=\left(u_{0,1}, \ldots, u_{0, N}\right), \eta \in X$ and $g \in$ $C^{1}([0, \tau] ; \mathbb{R})$ are given.

Theorem 13 allows to conclude that if $z, u_{0} \in \mathscr{D}(A)$ (in particular they belong to $H^{1}\left(\mathbb{R}^{n}\right),\left\langle\left\langle\eta, u_{0}\right\rangle\right\rangle=g(0)$ and $\langle\langle\eta, z\rangle\rangle \neq 0)$ the problems (319)-(321) admit a unique solution $(u, f) \in\left[C^{1}([0, \tau] ; X) \cap C([0, \tau] ; \mathscr{D}(A))\right] \times C([0, \tau] ; \mathbb{R})$.

As an example, consider the following identification problem related to Maxwell equations:

$$
\begin{gathered}
D_{t} E=-c \operatorname{rot} H+f(t) z, \quad(t, x) \in[0, \tau] \times \mathbb{R}^{3}, \\
D_{t} H=-c \operatorname{rot} E, \quad(t, x) \in[0, \tau] \times \mathbb{R}^{3}, \\
E(0, x)=E_{0}(x), \quad H(0, x)=H_{0}(x), \quad x \in \mathbb{R}^{3}, \quad \\
\left\langle\left\langle\eta^{1}, E(t, \cdot)\right\rangle\right\rangle+\left\langle\left\langle\eta^{2}, H(t, \cdot)\right\rangle\right\rangle=g(t), \quad t \in[0, \tau],
\end{gathered}
$$

where $c \in \mathbb{R}_{+}, z, E_{0}, H_{0}, \eta^{1}, \eta^{2} \in L^{2}\left(\mathbb{R}^{3}\right)^{3}, \operatorname{div} E_{0}=0$, $\operatorname{div} H_{0}=0$ and $\operatorname{div} z=0$ in $\mathbb{R}^{3}$, and $g \in C^{1}([0, \tau] ; \mathbb{R})$ are given.

We note that $\operatorname{div} E_{0}=0, \operatorname{div} H_{0}=0$, and $\operatorname{div} z=0$ in $\mathbb{R}^{3}$ imply $\operatorname{div} E(t, \cdot)=0$ and $\operatorname{div} H(t, \cdot)=0$ in $\mathbb{R}^{3}$, via $(322)$ and (323).

We notice that our assumption concerning $z$ and $E_{0}$ ensure that no electric charges occur in $\mathbb{R}^{3}$. 

that

Introduce now the vector $u=(E, H) \in L^{2}\left(\mathbb{R}^{3}\right)^{6}$. Recalling

$$
\begin{aligned}
\operatorname{rot} K=( & D_{x_{2}} K_{3}-D_{x_{3}} K_{2}, D_{x_{3}} K_{1} \\
& \left.\quad-D_{x_{1}} K_{3}, D_{x_{1}} K_{2}-D_{x_{2}} K_{1}\right),
\end{aligned}
$$

we observe that (322) and (323) can be rewritten in the unified form:

$$
\begin{array}{r}
D_{t} u=\mathscr{A}^{1} D_{x_{1}} u+\mathscr{A}^{2} D_{x_{2}} u+\mathscr{A}^{3} D_{x_{3}} u+f(t)\left[\begin{array}{l}
z \\
0
\end{array}\right], \\
(t, x) \in[0, \tau] \times \mathbb{R}^{3},
\end{array}
$$

where all the elements in $\mathscr{A}^{j}=\left(a_{h, k}^{j}\right)_{h, k=1}^{6}$ vanish but the following:

$$
\begin{array}{lll}
a_{2,6}^{1}=a_{6,2}^{1}=c, & a_{3,5}^{1}=a_{5,3}^{1}=-c, & a_{3,4}^{2}=a_{4,3}^{2}=c, \\
a_{1,6}^{2}=a_{6,1}^{2}=-c, & a_{1,5}^{3}=a_{5,1}^{3}=c, & a_{2,4}^{3}=a_{4,2}^{3}=-c .
\end{array}
$$

Define now

$$
\begin{array}{r}
X=\left\{u \in L^{2}\left(\mathbb{R}^{3}\right)^{6}: \operatorname{div}\left(u_{1}, u_{2}, u_{3}\right)\right. \\
\left.=\operatorname{div}\left(u_{4}, u_{5}, u_{6}\right)=0 \text { in } \mathbb{R}^{3}\right\}, \\
Y=\left\{u \in L^{2}\left(\mathbb{R}^{3}\right)^{6}: \operatorname{rot}\left(u_{1}, u_{2}, u_{3}\right)\right. \\
\left.=\operatorname{rot}\left(u_{4}, u_{5}, u_{6}\right)=0 \text { in } \mathbb{R}^{3}\right\} .
\end{array}
$$

We observe that it is easy to check that $X$ is a closed subspace in $L^{2}\left(\mathbb{R}^{3}\right)^{6}$ orthogonal to $Y$.

We observe now that, according to our assumptions, the initial value

$$
u_{0}=\left(E_{0}, H_{0}\right)
$$

belongs to $X$, while the additional condition can be expressed as

$$
\langle\langle\eta, u(t, \cdot)\rangle\rangle=g(t), \quad t \in[0, \tau],
$$

where $\eta=\left(\eta^{1}, \eta^{2}\right) \in L^{2}\left(\mathbb{R}^{3}\right)^{6}$.

Since operator $A$ is defined by (318), with $N=6$, it generates a $C_{0}$-semigroup in $X$. Moreover, $\mathscr{D}(A)=L^{2}\left(\mathbb{R}^{3} ;\right.$ rot $) \times$ $L^{2}\left(\mathbb{R}^{3} ;\right.$ rot $)$, where $L^{2}\left(\mathbb{R}^{3} ;\right.$ rot $)=\left\{k \in L^{2}\left(\mathbb{R}^{3}\right)^{3}: \operatorname{rot} k \in\right.$ $\left.L^{2}\left(\mathbb{R}^{3}\right)^{3}\right\}$. Consequently, Corollary 2.1 in [8] applies provided $z, \eta^{1}, \eta^{2} \in L^{2}\left(\mathbb{R}^{3} ;\right.$ rot $),\left\langle\left\langle\eta, u_{0}\right\rangle\right\rangle=g(0)$, and $\left\langle\left\langle\eta^{1}, z\right\rangle\right\rangle \neq 0$. Then the identification Problems (322)-(325) admit a unique solution $(E, H) \in\left[C^{1}([0, \tau] ; X) \cap C\left([0, \tau] ; L^{2}\left(\mathbb{R}^{3} ;\right.\right.\right.$ rot $) \times$ $\left.\left.L^{2}\left(\mathbb{R}^{3} ; \operatorname{rot}\right)\right)\right] \times C([0, \tau] ; \mathbb{R})$.

Remark 46. Likewise we could treat identification problems for the Dirac equation in nonrelativistic Quantum Mechanics (cf. [22, page 54]), making use of the function space $L^{2}\left(\mathbb{R}^{3}\right)^{4}$.
Application 5. Let $A$ be the linear operator defined in $X=$ $L^{2}\left(\mathbb{R}^{n}\right), n \in \mathbb{N} \backslash\{0\}$ by

$$
\begin{array}{r}
\mathscr{D}(A)=H^{2}\left(\mathbb{R}^{n}\right), \quad A u(x)=i \Delta u(x)-V(x) u(x), \\
u \in \mathscr{D}(A),
\end{array}
$$

where the potential $V$ belongs to $L^{p}\left(\mathbb{R}^{n} ; \mathbb{R}\right)$ for some $p>$ $n / 2$ and $p \geq 2$. Then it is well known (cf. [23]) that $A$ generates a group of unitary operators on $L^{2}\left(\mathbb{R}^{n}\right)$. Therefore, Proposition 35 yields the following result.

Corollary 47. Let $z, u_{0} \in H^{2}\left(\mathbb{R}^{n}\right), \eta \in L^{2}\left(\mathbb{R}^{n}\right),\langle\eta, z\rangle \neq 0$, $g \in C^{1}([0, \tau] ; \mathbb{C}),\left\langle\eta, u_{0}\right\rangle=g(0), V \in L^{p}\left(\mathbb{R}^{n} ; \mathbb{R}\right)$ for some $p>n / 2$ and $p \geq 2$. Then the identification problem

$$
\begin{gathered}
D_{t} u(t, x)=i \Delta u(t, x)-V(x) u(t, x)+f(t) z(x), \\
(t, x) \in[0, \tau] \times \mathbb{R}^{n}, \\
u(0, x)=u_{0}(x), \quad x \in \mathbb{R}^{n},
\end{gathered}
$$

$$
\int_{\mathbb{R}^{n}} \eta(x) u(t, x) d x=g(t), \quad t \in[0, \tau],
$$

admits a unique solution $(u, f) \in\left[C^{1}\left([0, \tau] ; L^{2}\left(\mathbb{R}^{n}\right)\right) \cap C([0\right.$, $\left.\left.\tau] ; H^{2}\left(\mathbb{R}^{n}\right)\right)\right] \times C([0, \tau] ; \mathbb{R})$.

Application 6. In a very recent paper [24] Taira exhibits a functional approach to the existence of Markov processes endowed with both Dirichlet and oblique derivative and the so-called first-order Wentzell boundary conditions for secondorder uniformly elliptic differential equations with discontinuous coefficients. More exactly, the related differential operator is assumed to be of the form

$$
\mathscr{A} u=\sum_{i, j}^{N} a^{i, j} D_{x_{i}} D_{x_{j}} u+\sum_{i, j}^{N} b^{i} D_{x_{i}} u+c(x) u
$$

on a bounded domain $\Omega \subset \mathbb{R}^{N}, N \geq 3$, with boundary $\partial \Omega$ of class $C^{1,1}$.

The discontinuous real-valued coefficients are assumed to satisfy the following properties:

(i) $a^{i, j} \in V M O(\Omega) \cap L^{\infty}(\Omega), a^{i, j}(x)=a^{j, i}(x)$ for a.e. $x \in$ $\Omega$ and $\lambda^{-1}|\xi|^{2} \leq \sum_{i, j}^{N} a^{i, j}(x) \xi_{i} \xi_{j} \leq \lambda|\xi|^{2}$ for a.e. $x \in \Omega$, all $\xi \in \mathbb{R}^{N}$ and some $\lambda \in[1,+\infty)$;

(ii) $b^{i} \in L^{\infty}(\Omega)$;

(iii) $c \in L^{\infty}(\Omega)$ and $c(x) \leq 0$ for a.e. $x \in \Omega$.

The differential operator $\mathscr{A}$ is a diffusion operator describing a strong Markov process with continuous paths in $\Omega$ such as Brownian motions. 
Consider also a boundary operator of the form

$$
\begin{array}{r}
L u(x)=\mu(x) D_{n} u(x)+\beta(x) \cdot \nabla_{\tau} u(x) \\
+\gamma(x) u(x)-\delta(x)(\mathscr{A} u)_{\partial \Omega}, \\
x \in \partial \Omega,
\end{array}
$$

where $\nabla_{\tau}$ denotes the tangential component of the gradient along $\partial \Omega$.

The following assumptions on the coefficients of $L$ are made:

(iv) $\mu$ is Lipschitz continuous on $\partial \Omega$ and $\mu(x) \geq 0$ for all $x \in \partial \Omega$;

(v) $\beta$ is a Lipschitz continuous vector field on $\partial \Omega$;

(vi) $\gamma$ is a Lipschitz continuous on $\partial \Omega$ and $\gamma(x) \leq 1$ for all $x \in \partial \Omega$;

(vii) $\delta$ is a Lipschitz continuous on $\partial \Omega$ and $\delta(x) \geq 0$ for all $x \in \partial \Omega$

(viii) $n(x)=\left(n_{1}(x), \ldots, n_{N}(x)\right)$ is the unit interior normal at $x$ to $\partial \Omega$.

The terms $\mu D_{n} u, \beta \cdot \nabla_{\tau} u, \gamma u, \delta(\mathscr{A} u)_{\partial \Omega}$ correspond, respectively, to the following phenomena: reflection, drift along the boundary, absorption, and sticking (or viscosity).

Let $C(\bar{\Omega})$ be the Banach space of real-valued functions continuous on $\bar{\Omega}$ endowed with the sup-norm.

Associated with the formal differential operator $\mathscr{A}$ we introduce the realization $A$ defined by

$$
\mathscr{D}(A)=\{u \in C(\bar{\Omega}): \mathscr{A} u \in C(\bar{\Omega}), L u=0 \text { on } \partial \Omega\} .
$$

Functions in $\mathscr{D}(A)$ are said to satisfy Wentzell conditions of the first order.

Finally, recall that a strongly continuous semigroup $\{T(t)\}_{t \geq 0}$ on $C(\bar{\Omega})$ is called a Feller semigroup if it satisfies

$$
\begin{array}{r}
f \in C(\bar{\Omega}), \quad 0 \leq f(x) \leq 1, \\
x \in \bar{\Omega} \text { implies } 0 \leq(T(t) f)(x) \leq 1, \quad x \in \bar{\Omega} .
\end{array}
$$

This implies that $\{T(t)\}_{t \geq 0}$ is nonnegative and contracting on $C(\bar{\Omega})$.

Assume now that $p \in(N,+\infty)$ and $A: \mathscr{D}(A) \subset C(\bar{\Omega}) \rightarrow$ $C(\bar{\Omega})$ is defined by

$$
\begin{gathered}
\mathscr{D}(A)=\left\{u \in W^{2, p}(\Omega): \mathscr{A} u \in C(\bar{\Omega}), L u=0 \text { on } \partial \Omega\right\}, \\
A u=\mathscr{A} u, \\
u \in \mathscr{D}(A) .
\end{gathered}
$$

Then Theorem 1.1 in [24, page 717] establishes that $A$ generates a Feller semigroup on $C(\bar{\Omega})$. Therefore, Theorem 13 applies. Recall now that the dual $C(\bar{\Omega})^{*}$ consists of real measure $v$ with finite variation coinciding with $\|\mu\|_{C(\bar{\Omega})}$.
Finally, consider the identification problem:

$$
\begin{gathered}
D_{t} u(t, x)=\mathscr{A} u(t, x)+f(t) z(x), \quad(t, x) \in[0, \tau] \times \Omega, \\
u(0, x)=u_{0}(x), \quad x \in \mathbb{R}^{n}, \\
L u(t, x)=0, \quad(t, x) \in[0, \tau] \times \partial \Omega, \\
\int_{\Omega} u(t, x) v(d x)=g(t), \quad t \in[0, \tau] .
\end{gathered}
$$

If $z, u_{0} \in \mathscr{D}(A), g \in C^{1}([0, \tau] ; \mathbb{R}), \int_{\Omega} u_{0}(x) \nu(d x)=g(0)$, $\int_{\Omega} z(x) v(d x) \neq 0$, then the previous Problems admit a unique solution $(u, f) \in\left[C^{1}([0, \tau] ; C(\bar{\Omega})) \cap C([0, \tau] ; \mathscr{D}(A))\right] \times$ $C([0, \tau] ; \mathbb{R})$.

Application 7. We are here again concerned with the OrnsteinUhlenbeck operator, but in $L^{p}\left(\mathbb{R}^{n}\right)$. For this purpose denote by $\widetilde{\mathscr{L}}_{p}, p \in(1,+\infty)$, the realization of the the OrnsteinUhlenbeck operator $\mathscr{A}$. Consider now the identification problem:

$$
\begin{gathered}
D_{t} u(t, x)=\widetilde{\mathscr{L}}_{p} u(t, x)+f(t) z(x), \quad(t, x) \in[0, \tau] \times \mathbb{R}^{n}, \\
u(0, x)=u_{0}(x), \quad x \in \mathbb{R}^{n}, \\
\int_{\Omega} u(t, x) d x=g(t), \quad t \in[0, \tau],
\end{gathered}
$$

In view of [11, Proposition 9.4.2, page 280] operator $\widetilde{\mathscr{L}}$ defined by

$$
\begin{gathered}
\mathscr{D}\left(\widetilde{\mathscr{L}}_{p}\right)=\left\{u \in W^{2, p}(\Omega): \mathscr{A} u \in L^{p}\left(\mathbb{R}^{n}\right)\right\}, \\
\widetilde{\mathscr{L}}_{p} u=\mathscr{A} u, \\
u \in \mathscr{D}\left(\widetilde{\mathscr{L}}_{p}\right),
\end{gathered}
$$

generates a strongly continuous semigroup $\left\{T_{p}(t)\right\}_{t \geq 0}$, which is not analytic.

In this case, we can apply Theorem 13. Indeed, if $z, u_{0} \in$ $\mathscr{D}\left(\widetilde{\mathscr{L}}_{p}\right), \eta \in L^{p^{\prime}}\left(\mathbb{R}^{n}\right),(1 / p)+\left(1 / p^{\prime}\right)=1, g \in C^{1}([0, \tau]$; $\mathbb{R}), \int_{\Omega} \eta(x) u_{0}(x) d x=g(0), \int_{\Omega} \eta(x) z(x) d x \neq 0$, then the previous Problems admit a unique solution $(u, f) \in\left[C^{1}([0\right.$, $\left.\left.\tau] ; L^{p}\left(\mathbb{R}^{n}\right)\right) \cap C\left([0, \tau] ; \mathscr{D}\left(\widetilde{\mathscr{L}}_{p}\right)\right)\right] \times C([0, \tau] ; \mathbb{R})$.

\section{Appendices}

\section{A. Perturbing Generators: An Interpolation Approach}

Let $X$ be a complex Banach space with norm $\|\cdot\|$.

We make the following assumptions, where $\rho(L)$ denotes the resolvent set of a linear closed operator $L: \mathscr{D}(L) \subset X \rightarrow$ $X$ :

(H1) $A: \mathscr{D}(A) \subset X \rightarrow X$ is a (possibly multivalued) linear closed operator, whose resolvent $\rho(A)$ contains 
the set $\Sigma_{\alpha}=\left\{\lambda \in \mathbb{C}: \operatorname{Re} \lambda \geq-c_{0}(1+|\operatorname{Im} \lambda|)^{\alpha}\right\}, c_{0}>0$, $0<\beta \leq \alpha \leq 1$;

(H2) $\left\|(\lambda-A)^{-1}\right\|_{\mathscr{L}(X)} \leq C_{0}(1+|\lambda|)^{-\beta}, C_{0}>0$, and $\beta \in(0,1]$, for all $\lambda \in \Sigma_{\alpha}$;

(H3) $B \in \mathscr{L}\left(\mathscr{D}(A) ; X_{A}^{\theta}\right)$ for some $\theta \in(1-\beta, 1)$,

where the space $X_{A}^{\theta}$, introduced in [3], is defined by $X_{A}^{\theta}=$ $\left\{u \in X: \sup _{t>0} t^{\theta}\left\|A(t-A)^{-1}\right\|<+\infty\right\}$.

We want to show that the linear closed operator $\widetilde{A}=A+B$ generates a $C^{\infty}$-semigroup if $\beta \in(0,1)$ (resp., an analytical semigroup, if $\beta=1)\left\{e^{t \widetilde{A}_{t>0}}\right.$ of linear bounded operators in $\mathscr{L}(X)$. For this purpose, we extend two perturbation results listed in [20].

For all $f \in X$ and $\lambda \in \lambda_{0}+\Sigma_{\alpha}$, consider the spectral equation:

$$
\lambda u-A u-B u=f, \quad u \in \mathscr{D}(A) .
$$

Applying operator $(\lambda-A)^{-1}$ to both sides in (A.1), we deduce the following equation:

$$
\left[I-(\lambda-A)^{-1} B\right] u=(\lambda-A)^{-1} f, \quad u \in \mathscr{D}(A) .
$$

Recall now (cf. [3, page 49]) that

$$
\begin{aligned}
\left\|A(\lambda-A)^{-1} x\right\| & \leq c|\lambda|^{1-\beta-\theta}\|x\|_{X_{A}^{\theta}}, \\
\lambda & \in \lambda_{0}+\Sigma_{\alpha}, \quad x \in X_{A}^{\theta} .
\end{aligned}
$$

Consider now the following resolvent identity:

$$
\begin{aligned}
A^{\circ}(\lambda-A)^{-1}= & A(|\lambda|-A)^{-1} \\
& -(\lambda-|\lambda|)(\lambda-A)^{-1} A(|\lambda|-A)^{-1}, \\
& \lambda,|\lambda| \in \rho(A) .
\end{aligned}
$$

If $x \in X_{A}^{\theta}$, we get the estimate

$$
\begin{aligned}
& \left\|A(\lambda-A)^{-1} x\right\| \\
& \quad \leq\left[c(1+|\lambda|)^{-\theta}+2 C_{0}|\lambda|(1+|\lambda|)^{-\beta}(1+|\lambda|)^{-\theta}\right]\|x\|_{X_{A}^{\theta}} \\
& \quad \leq \widetilde{c}(1+|\lambda|)^{1-\beta-\theta}\|x\|_{X_{A}^{\theta}}, \quad \lambda \in \Sigma_{\alpha}, \quad x \in X_{A}^{\theta} .
\end{aligned}
$$

Since $x \rightarrow\|A x\|$ is an equivalent norm on $\mathscr{D}(A)$, indeed $0 \in$ $\rho(A)$, we conclude that

$$
\left\|(\lambda-A)^{-1}\right\|_{\mathscr{L}\left(X_{A}^{\theta} ; \mathscr{D}(A)\right)} \leq \widetilde{\mathcal{c}}(1+|\lambda|)^{1-\beta-\theta}, \quad \theta \in(1-\beta, 1) .
$$

Since $B \in \mathscr{L}\left(X_{A}^{\theta} ; \mathscr{D}(A)\right)$, we deduce the estimate

$$
\begin{aligned}
& \left\|(\lambda-A)^{-1} B\right\|_{\mathscr{L}(\mathscr{D}(A))} \\
& \quad \leq\left\|(\lambda-A)^{-1}\right\|_{\mathscr{L}\left(X_{A}^{\theta} ; \mathscr{D}(A)\right)}\|B\|_{\mathscr{L}\left(\mathscr{D}(A) ; X_{A}^{\theta}\right)} \\
& \quad \leq C_{1}(1+|\lambda|)^{1-\beta-\theta}\|B\|_{\mathscr{L}\left(\mathscr{D}(A) ; X_{A}^{\theta}\right), \quad \lambda \in \Sigma_{\alpha} .}
\end{aligned}
$$

This implies

$$
\left\|(\lambda-A)^{-1} B\right\|_{\mathscr{L}(\mathscr{D}(A))} \leq \frac{1}{2}, \quad \lambda \in \Sigma_{\alpha, \beta, \theta},
$$

where, recall that, owing to (H3), $\beta+\theta>1$,

$$
\Sigma_{\alpha, \beta, \theta}=\left\{\lambda \in \Sigma_{\alpha}:(1+|\lambda|)^{\beta+\theta-1}>C_{1}\|B\|_{\mathscr{L}\left(\mathscr{D}(A) ; X_{A}^{\theta}\right)}\right\} .
$$

Then, according to (A.8), we deduce that $I+(\lambda-A)^{-1} B$ is invertible in $\mathscr{D}(A)$ and, for all $\lambda \in \Sigma_{\alpha, \beta, \theta}$, its inverse is estimated by

$$
\left\|\left[I+(\lambda-A)^{-1} B\right]^{-1}\right\|_{\mathscr{L}(\mathscr{D}(A))} \leq 2, \quad \lambda \in \Sigma_{\alpha, \beta, \theta} .
$$

Hence (A.2) admits, for all $\lambda \in \Sigma_{\alpha, \beta, \theta}$ and $f \in X$, the unique solution:

$$
u=\left[I-(\lambda-A)^{-1} B\right]^{-1}(\lambda-A)^{-1} f \in \mathscr{D}(A)
$$

satisfying the estimate

$$
\|u\|_{\mathscr{D}(A)} \leq 2\left\|A(\lambda-A)^{-1} f\right\| \leq C(1+|\lambda|)^{1-\beta}\|f\| .
$$

Therefore, for all $\lambda \in \Sigma_{\alpha, \beta, \theta}$ the operator $\lambda-A-B$ has a bounded inverse from $X$ into $\mathscr{D}(A)$, and, hence, from $X$ into itself.

We now estimate $u$ in $X$. From the equation $\lambda u=A u+$ $B u+f=A u+B A^{-1} A u+f$, for all $\lambda \in \Sigma_{\alpha, \beta, \theta}$, we get

$$
\begin{aligned}
|\lambda|\|u\| & \leq\left(1+\left\|B A^{-1}\right\|_{\mathscr{L}(X)}\right)\|A u\|+\|f\| \\
& =\left(1+\left\|B A^{-1}\right\|_{\mathscr{L}(X)}\right)\|u\|_{\mathscr{D}(A)}+\|f\| \\
& \leq\left[C\left(1+\left\|B A^{-1}\right\|_{\mathscr{L}(X)}\right)(1+|\lambda|)^{1-\beta}+1\right]\|f\| \\
& \leq C^{\prime}(1+|\lambda|)^{1-\beta}\|f\| .
\end{aligned}
$$

As a consequence, from (A.13) we deduce the desired estimate

$$
\|u\| \leq C(1+|\lambda|)^{-\beta}\|f\|, \quad \lambda \in \Sigma_{\alpha, \beta, \theta} .
$$

\section{B. A General Approach to the Identification Problem}

Let $X, \mathscr{F}$, and $\mathscr{Z}$ be three complex Banach spaces with norms $\|\cdot\|,\|\cdot\|_{\mathscr{F}},\|\cdot\|_{\mathscr{T}}$, respectively.

We make the following assumptions, where $\rho(L)$ denotes the resolvent set of a linear closed operator $L: \mathscr{D}(L) \subset X \rightarrow$ $X$ :

(H1) $A: \mathscr{D}(A) \subset X \rightarrow X$ is a linear closed operator, whose resolvent $\rho(A)$ contains the set $\Sigma_{\alpha}=\{\lambda \in \mathbb{C}$ : $\left.\operatorname{Re} \lambda \geq-c_{0}(1+|\operatorname{Im} \lambda|)^{\alpha}\right\}, c_{0}>0$, and $\alpha \in(0,1]$;

(H2) $\left\|(\lambda-A)^{-1}\right\|_{\mathscr{L}(X)} \leq C_{0}(1+|\lambda|)^{-\beta}, C_{0}>0$, and $\beta \epsilon$ $(0, \alpha]$, for all $\lambda \in \Sigma_{\alpha}$; 
(H3) $M \in \mathscr{B}(\mathscr{F} \times \mathscr{Z} ; X), \mathscr{B}(\mathscr{F} \times \mathscr{Z} ; X)$ standing for the Banach space of all linear bounded sesquilinear operators from $\mathscr{F} \times \mathscr{Z}$ to $X$;

(H4) $\Phi \in \mathscr{L}(X ; \mathscr{F})$;

(H5) for each fixed $Z \in \mathscr{Z}$ and for all $H \in \mathscr{F}$ the equation $\Phi[M(F, Z)]=H$ is uniquely solvable in $\mathscr{F}$ and its solution can be represented by $F=\Psi[H, Z]$, where the (nonlinear) operator $\Psi: \mathscr{D}(\Psi) \subset \mathscr{F} \times \mathscr{Z} \rightarrow$ $\mathscr{F}$ is linear continuous as a function of $H$; that is $\|\Psi[H, Z]\|_{\mathscr{F}} \leq C_{1}(Z)\|H\|_{\mathscr{F}}$, for all $H \in \mathscr{F} ;$

(H6) there exist Banach spaces $X_{A}^{\theta}$ and $\mathscr{Z}_{A}^{\theta}$ in $X$ and $\mathscr{Z}$, respectively, with $\theta>1-\beta$, such that

$$
\|M(F, Z)\|_{X_{A}^{\theta}} \leq C(\theta)\|F\|_{\mathscr{F}}\|Z\|_{\mathscr{E}^{\theta}}, \quad(F, Z) \in \mathscr{F} \times \mathscr{Z}^{\theta} .
$$

We consider now the following problem: determine a pair of functions $u:[0, \tau] \rightarrow X$ and $F:[0, \tau] \rightarrow \mathscr{F}$ such that

$$
\begin{gathered}
u^{\prime}(t)-A u(t)=M(F(t), Z)+g(t), \quad 0 \leq t \leq \tau, \\
u(0)=u_{0}, \\
\Phi[u(t)]=H(t), \quad 0 \leq t \leq \tau,
\end{gathered}
$$

where $u_{0} \in \mathscr{D}(A), g \in C([0, \tau] ; X), H \in C^{1}([0, \tau] ; \mathscr{F})$.

Apply now operator $\Phi$ to both sides of our differential equation. Using assumptions (H4), we obtain the following equation:

$$
\begin{aligned}
& H^{\prime}(t)-\Phi[A u(t)] \\
& \quad=\Phi[M(F(t), Z)]+\Phi[g(t)], \quad 0 \leq t \leq \tau .
\end{aligned}
$$

From assumptions (H3) and (H5) we deduce

$$
F(t)=\Psi\left[H^{\prime}(t), Z\right]-\Psi[\Phi[g(t)], Z]-\Psi[\Phi[A u(t)], Z],
$$$$
0 \leq t \leq \tau \text {. }
$$

Inserting this expression into the differential equation (B.2), we conclude that the identification Problem (B.2) is equivalent to the unusual following Cauchy problem:

$$
\begin{gathered}
u^{\prime}(t)-A u(t)+M(\Psi[\Phi[A u(t)], Z], Z) \\
=M\left(\Psi\left[H^{\prime}(t), Z\right], Z\right) \\
-M(\Psi[\Phi[g(t)], Z], Z)+g(t), \quad 0 \leq t \leq \tau, \\
u(0)=u_{0} .
\end{gathered}
$$

Introduce now the linear operator $B$ defined by

$$
\mathscr{D}(B)=\mathscr{D}(A), \quad B u=-M(\Psi[\Phi[A u], Z], Z),
$$

$$
u \in \mathscr{D}(B) \text {. }
$$

From assumptions (H5) and (H6) we deduce the bounds as follows:

$$
\begin{aligned}
\|B u\|_{X_{A}^{\theta}} & \leq C(\theta)\|\Psi[\Phi[A u], Z]\|_{\mathscr{F}}\|Z\|_{\mathscr{Z}^{\theta}} \\
& \leq C(\theta) C_{1}(Z)\|\Phi[A u]\|_{\mathscr{F}}\|Z\|_{\mathscr{Z}^{\theta}} \\
& \leq C(\theta) C_{1}(Z)\|\Phi\|_{\mathscr{L}(X, F)}\|A u\|_{X}\|Z\|_{\mathscr{L}^{\theta}} \\
& \leq C(\theta) C_{1}(Z)\|\Phi\|_{\mathscr{L}(X, F)}\|Z\|_{\mathscr{Z}^{\theta}}\|u\|_{\mathscr{D}(A)} .
\end{aligned}
$$

Consequently, the linear operator $A+B$, with $\mathscr{D}(A+B)=$ $\mathscr{D}(A)$, generates a $C^{\infty}$-semigroup in $X$ if $\beta \in(0,1)$ (resp., an analytical semigroup, if $\beta=1)\left\{e^{t \widetilde{A}}\right\}_{t>0}$ of linear bounded operators in $X$ with

$$
\left\|(\lambda-A-B)^{-1}\right\|_{\mathscr{L}(X)} \leq C(1+|\lambda|)^{-\beta}, \quad \lambda \in \Sigma_{\alpha}, \lambda \text { large. }
$$

Remark 48. Note that no compactness is required to operator $M(\Psi[\Phi[A u], Z], Z)$ in order that the perturbed operator generates a $C^{\infty}$-(or an analytical) semigroup.

Application 8. In the case of Application $8 B$ is given by

$$
B u=-M(\Psi[\Phi[A u], Z], Z)=-\sum_{j, k=1}^{N} \widetilde{\Psi}_{j, k}(Z) \Phi_{k}[A u] z_{j}
$$

In this case one has

$$
X_{A}^{\theta} \subset X_{A+B}^{\theta}
$$

Proof. Suppose $f \in X$ and $u=(t+A+B)^{-1} f$. Then $(t+$ A) $u=f-B u$. Hence,

$$
\begin{aligned}
A u & =A(t+A)^{-1} f-A(t+A)^{-1} B u \\
& =A(t+A)^{-1} f+\sum_{j, k=1}^{N} \widetilde{\Psi}_{j, k}(Z) \Phi_{k}[A u] A(t+A)^{-1} z_{j} .
\end{aligned}
$$

Therefore,

$\|A u\|_{X}$

$$
\begin{aligned}
& \leq\left\|k A(t+A)^{-1} f\right\|_{X} \\
& +\sum_{j, k=1}^{N}\left|\widetilde{\Psi}_{j, k}(Z)\right|\left\|\Phi_{k}\right\|_{X^{*}}\|A u\|_{X}\left\|A(t+A)^{-1} z_{j}\right\|_{X} \\
& \leq\left\|A(t+A)^{-1} f\right\|_{X}+t^{-\theta} \sum_{j, k=1}^{N}\left|\widetilde{\Psi}_{j, k}(Z)\right|\left\|\Phi_{k}\right\|_{X^{*}}\|A u\|_{X}\left\|z_{j}\right\|_{X_{A}^{\theta}} .
\end{aligned}
$$

This implies

$$
\begin{aligned}
& \left(1-t^{-\theta} \sum_{j, k=1}^{N}\left|\widetilde{\Psi}_{j, k}(Z)\right|\left\|\Phi_{k}\right\|_{X^{*}}\left\|z_{j}\right\|_{X_{A}^{\theta}}\right)\|A u\|_{X} \\
& \leq\left\|A(t+A)^{-1} f\right\|_{X} .
\end{aligned}
$$


Hence, if $t$ is sufficiently large,

$$
\|A u\|_{X} \leq \frac{\left\|A(t+A)^{-1} f\right\|_{X}}{1-t^{-\theta} \sum_{j, k=1}^{N}\left|\widetilde{\Psi}_{j, k}(Z)\right|\left\|\Phi_{k}\right\|_{X^{*}}\left\|z_{j}\right\|_{X_{A}^{\theta}}} .
$$

On the other hand,

$$
\begin{aligned}
\|B u\|_{X} \leq & \sum_{j, k=1}^{N}\left|\widetilde{\Psi}_{j, k}(Z)\right|\left\|\Phi_{k}\right\|_{X^{*}}\|A u\|_{X}\left\|z_{j}\right\|_{X} \\
\leq & \frac{\sum_{j, k=1}^{N}\left|\widetilde{\Psi}_{j, k}(Z)\right|\left\|\Phi_{k}\right\|_{X^{*}}\left\|z_{j}\right\|_{X}}{1-t^{-\theta} \sum_{j, k=1}^{N}\left|\widetilde{\Psi}_{j, k}(Z)\right|\left\|\Phi_{k}\right\|_{X^{*}}\left\|z_{j}\right\|_{X_{A}^{\theta}}} \\
& \times\left\|A(t+A)^{-1} f\right\|_{X} .
\end{aligned}
$$

Consequently,

$$
\begin{aligned}
& \left\|(A+B)(t+A+B)^{-1} f\right\|_{X} \\
& =\|(A+B) u\|_{X} \leq \frac{1+\sum_{j, k=1}^{N}\left|\widetilde{\Psi}_{j, k}(Z)\right|\left\|\Phi_{k}\right\|_{X^{*}}\left\|z_{j}\right\|_{X}}{1-t^{-\theta} \sum_{j, k=1}^{N}\left|\widetilde{\Psi}_{j, k}(Z)\right|\left\|\Phi_{k}\right\|_{X^{*}}\left\|z_{j}\right\|_{X_{A}^{\theta}}} \\
& \quad \times\left\|A(t+A)^{-1} f\right\|_{X} .
\end{aligned}
$$

Therefore, one concludes that there exists a constant $c>0$ such that

$$
\begin{aligned}
& \sup _{t \geq c} t^{\theta}\left\|A(t+A)^{-1} f\right\|_{X}<\infty \\
& \Longrightarrow \sup _{t \geq c} t^{\theta}\left\|(A+B)(t+A+B)^{-1} f\right\|_{X}<\infty .
\end{aligned}
$$

We consider the problem

$$
\begin{aligned}
& u^{\prime}(t)-A u(t)+M(\Psi[\Phi[A u(t)], Z], Z) \\
&=M\left(\Psi\left[H^{\prime}(t), Z\right], Z\right)-M(\Psi[\Phi[g(t)], Z], Z)+g(t), \\
& 0 \leq t \leq \tau,
\end{aligned}
$$

$$
u(0)=u_{0}
$$

Suppose that $A u_{0} \in X_{A}^{\theta}, 3-\alpha-\beta-\alpha \beta<\theta<1$, and

$$
\begin{aligned}
M & \left(\Psi\left[H^{\prime}(\cdot), Z\right], Z\right) \\
& -M(\Psi[\Phi[g(\cdot)], Z], Z) \\
& +g \in C([0, \tau] ; X) \cap B\left([0, \tau] ; X_{A}^{\theta}\right) .
\end{aligned}
$$

Since $R(B) \subset X_{A}^{\theta}$, one has $(A+B) u_{0} \in X_{A}^{\theta}$. In view of (B.9) one observes

$$
\begin{gathered}
(A+B) u_{0} \in X_{A+B}^{\theta}, \\
M\left(\Psi\left[H^{\prime}(\cdot), Z\right], Z\right) \\
-M(\Psi[\Phi[g(\cdot)], Z], Z) \\
+g \subset C([0, \tau] ; X) \cap B\left([0, \tau] ; X_{A+B}^{\theta}\right) .
\end{gathered}
$$

By virtue of Corollary 3.3 of [25] problem (B.18) one admits a unique solution such that

$$
\begin{aligned}
& u^{\prime} \in C([0, \tau] ; X) \cap B\left([0, \tau] ; X_{A+B}^{(\theta-3+2 \alpha+\beta) / \alpha}\right) \\
& \quad \subset C([0, \tau] ; X) \cap B\left([0, \tau] ; X_{A}^{(\theta-3+\alpha+\beta+\alpha \beta) / \alpha}\right),
\end{aligned}
$$

$$
\begin{aligned}
&(A+B) u \in C^{(\theta-3+2 \alpha+\beta) / \alpha}([0, \tau] ; X) \cap B\left([0, \tau] ; X_{A+B}^{(\theta-3+2 \alpha+\beta) / \alpha}\right) \\
& C C^{(\theta-3+2 \alpha+\beta) / \alpha}([0, \tau] ; X) \cap B\left([0, \tau] ; X_{A}^{(\theta-3+\alpha+\beta+\alpha \beta) / \alpha}\right) .
\end{aligned}
$$

Let $k>0$ be so large that $A+B+k$ has a bounded inverse. Since by virtue of (B.22) and (B.23)

$$
(A+B+k) u \in C^{(\theta-3+2 \alpha+\beta) / \alpha}([0, \tau] ; X),
$$

one obtains

$$
A u=A(A+B+k)^{-1}(A+B+k) u \in C^{(\theta-3+2 \alpha+\beta) / \alpha}([0, \tau] ; X) .
$$

It follows from

$$
\begin{aligned}
s^{\theta} & \left\|A(s+A)^{-1} B u(t)\right\|_{X} \\
& =s^{\theta}\left\|\sum_{j, k=1}^{N} \widetilde{\Psi}_{j, k}(Z) \Phi_{k}[A u(t)] A(s+A)^{-1} z_{j}\right\|_{X} \\
& \leq \sum_{j, k=1}^{N}\left|\widetilde{\Psi}_{j, k}(Z)\right|\left\|\Phi_{k}\right\|_{X^{*}}\|A u(t)\|_{X} s^{\theta}\left\|A(s+A)^{-1} z_{j}\right\|_{X}
\end{aligned}
$$

that

$$
\|B u(t)\|_{X_{A}^{\theta}} \leq \sum_{j, k=1}^{N}\left|\widetilde{\Psi}_{j, k}(Z)\right|\left\|\Phi_{k}\right\|_{X^{*}}\|A u(t)\|_{X}\left\|z_{j}\right\|_{X_{A}^{\theta}} .
$$

Hence, with the aid of (B.25), one obtains

$$
B u \in B\left([0, \tau] ; X_{A}^{\theta}\right) .
$$

Since $0<2-\beta-\alpha \beta$, one has

$$
\theta(1-\alpha) \leq 1-\alpha<3-\alpha-\beta-\alpha \beta .
$$

This implies

$$
\frac{(\theta-3+\alpha+\beta+\alpha \beta)}{\alpha<\theta} .
$$

Hence, in view of (B.28), one has

$$
B u \in B\left([0, \tau] ; X_{A}^{(\theta-3+\alpha+\beta+\alpha \beta) / \alpha}\right) .
$$

From this and (B.23) it follows that

$$
A u \in B\left([0, \tau] ; X_{A}^{(\theta-3+\alpha+\beta+\alpha \beta) / \alpha}\right) .
$$


Thus, the unique solution to problem (B.18) satisfies

$$
\begin{aligned}
& u^{\prime} \in C([0, \tau] ; X) \cap B\left([0, \tau] ; X_{A}^{(\theta-3+\alpha+\beta+\alpha \beta) / \alpha}\right) \\
& A u \in C^{(\theta-3+2 \alpha+\beta) / \alpha}([0, \tau] ; X) \cap B\left([0, \tau] ; X_{A}^{(\theta-3+\alpha+\beta+\alpha \beta) / \alpha}\right) .
\end{aligned}
$$

It is also possible to show that $u \in B([0, \tau]$; $\left.X_{A}^{(\theta-3+\alpha+\beta+\alpha \beta) / \alpha}\right)$.

Application 9. Let $\mathscr{F}=l^{2}(\mathbb{R}), \mathscr{Z}=l^{2}(X), \mathscr{Z}^{\theta}=l^{2}\left(X_{A}^{\theta}\right), F=$ $\left\{f_{j}\right\}_{j=1}^{+\infty} \in l^{2}(\mathbb{R}), Z=\left\{z_{j}\right\}_{j=1}^{+\infty} \in \mathscr{Z}^{\theta}, M(F, Z)=\sum_{j=1}^{+\infty} f_{j} z_{j}$. Let $\Phi=\left\{\Phi_{j}\right\}_{j=1}^{+\infty} \in l^{2}\left(X^{*}\right)$ such that $\sum_{j, k=1}^{+\infty}\left|\Phi_{k}\left[z_{j}\right]\right|^{2}<1$. Therefore, equation $\Phi[M(F, Z)]=H$, with $H \in l^{2}(\mathbb{R})$, means

$$
\Phi\left(\sum_{j=1}^{+\infty} f_{j} z_{j}\right)=\left(\sum_{j=1}^{+\infty} f_{j} \Phi_{k}\left[z_{j}\right]\right)_{k \in \mathbb{N} \backslash\{0\}} .
$$

Suppose that the infinite matrix $\left(\Phi_{k}\left[z_{j}\right]\right)_{j, k=1}^{+\infty}$ defines an invertible operator in $\mathscr{L}\left(l^{2}(\mathbb{R})\right)$, the inverse of which is denoted by $\widetilde{\Psi}(Z)$, so that $F=\widetilde{\Psi}(Z) H=\widetilde{\Psi}(H, Z)$.

One also has

$$
\begin{aligned}
M(\Psi[\Phi[A u], Z], Z) & =\sum_{j=1}^{+\infty}(\widetilde{\Psi}(Z) \Phi[A u])_{j} z_{j} \\
& =\sum_{j, k=1}^{+\infty} \widetilde{\Psi}_{j, k}(Z) \Phi_{k}[A u] z_{j}
\end{aligned}
$$

Moreover,

$$
\begin{aligned}
& \|M(F, Z)\|_{X_{A}^{\theta}} \\
& \leq \sum_{j=1}^{+\infty}\left|f_{j}\right|\left\|z_{j}\right\|_{X_{A}^{\theta}} \leq\|F\|_{l^{2}(\mathbb{R})}\left(\sum_{j=1}^{+\infty}\left\|z_{j}\right\|_{X_{A}^{\theta}}^{2}\right)^{1 / 2} \\
& =:\|F\|_{l^{2}(\mathbb{R})}\|Z\|_{\mathscr{Z}^{\theta}}, \quad(F, Z) \in l^{2}(\mathbb{R}) \times \mathscr{Z}^{\theta} .
\end{aligned}
$$

Therefore, all assumptions (H1)-(H6) are verified. Consequently, we conclude that the linear operator $\widetilde{A} u=$ $-A u+\sum_{j=1}^{+\infty}(\widetilde{\Psi}(Z))_{j, k} \Phi_{k}[A u] z_{j}, u \in \mathscr{D}(A)$, generates a $C^{\infty}$ semigroup if $\beta \in(0,1)$ and $\theta \in(1-\beta, 1)$ (resp., an analytical semigroup, if $\beta=1$ and $\theta \in(0,1))$.

Reasoning as in Application 8, we conclude that if $\alpha+2 \beta+\alpha \beta>3, \alpha+\beta>3 / 2,4-\alpha-2 \beta-$ $\alpha \beta<\theta<1$, then the identification Problem (B.40), with $N=+\infty$, admits a unique solution $\left(u,\left\{f_{j}\right\}_{j \in \mathbb{N} \backslash\{0\}}\right)$ such that $u \in C^{1}([0, \tau] ; X) \cap B\left([0, \tau] ; X_{A}^{-1+[\alpha \beta+\theta-2(2-\alpha-\beta)] / \alpha}\right)$, $A u \in C^{[\theta-2(2-\alpha-\beta)] / \alpha}([0, \tau] ; X) \cap B\left([0, \tau] ; X_{A}^{-1+[\alpha \beta+\theta-2(2-\alpha-\beta)] / \alpha}\right)$, $f_{j} \in C^{[\theta-2(2-\alpha-\beta)] / \alpha}\left([0, \tau] ; l^{2}(\mathbb{R})\right), j \in \mathbb{N} \backslash\{0\}$.

Application 10. We consider here the following identification problem: determine $n(1+N)$ functions $u_{j}:[0, \tau] \rightarrow X$, $j=1, \ldots, n$, and $f_{j, k}:[0, \tau] \rightarrow \mathbb{R}, j=1, \ldots, n, k=1, \ldots, N$ such that

$$
\begin{gathered}
u_{j}^{\prime}(t)-A_{j} u_{j}(t)-B_{j}\left(u_{1}(t), \ldots, u_{n}(t)\right) \\
=\sum_{l=1}^{N} f_{j, l}(t) z_{l}+g_{j}(t), \quad 0 \leq t \leq \tau, j=1, \ldots, n, \\
u_{j}(0)=u_{0, j}, \quad j=1, \ldots, n,
\end{gathered}
$$

$\Phi_{k}\left[u_{j}(t)\right]=h_{j, k}(t), \quad 0 \leq t \leq \tau, j=1, \ldots, n, k=1, \ldots, N$.

Before stating precise properties involving operators, functionals and functions in (B.37) we apply (formally) functional $\Phi_{k}$ to both sides in the differential equation in (B.37). We obtain the following equations for all $t \in[0, \tau]$, $j=1, \ldots, n, k=1, \ldots, N$ :

$$
\begin{aligned}
& h_{j, k}^{\prime}(t)-\Phi_{k}\left[A_{j} u_{j}(t)\right]-\Phi_{k}\left[B_{j}\left(u_{1}(t), \ldots, u_{n}(t)\right)\right] \\
& -\Phi_{k}\left[g_{j}(t)\right]=\sum_{l=1}^{N} f_{j, l}(t) \Phi_{k}\left[z_{l}\right] .
\end{aligned}
$$

Suppose that $\left(\Phi_{k}\left[z_{l}\right]\right)_{l, k=1}^{N}$ is an invertible matrix and denote by $\widetilde{\Psi}(Z)=\left(\widetilde{\Psi}_{k, l}(Z)\right)_{k, l=1}^{N}$ its inverse.

Then we can solve system (B.38) for $\left(f_{j, l}(t)\right)_{j=1, l=1}^{n, N}$. For all $t \in[0, \tau], j=1, \ldots, n, l=1, \ldots, N$ we obtain

$$
\begin{aligned}
f_{j, l}(t)=\sum_{k=1}^{N}\left\{h_{j, k}^{\prime}(t)-\Phi_{k}\left[A_{j} u_{j}(t)\right]\right. \\
-\Phi_{k}\left[B_{j}\left(u_{1}(t), \ldots, u_{n}(t)\right)\right] \\
\left.-\Phi_{k}\left[g_{j}(t)\right]\right\} \widetilde{\Psi}_{k, l}(Z) .
\end{aligned}
$$

Consequently, our identification problem is equivalent to the following direct problem

$$
\begin{aligned}
& u_{j}^{\prime}(t)-A_{j} u_{j}(t)+\sum_{k, l=1}^{N} \Phi_{k}\left[A_{j} u_{j}(t)\right] \widetilde{\Psi}_{k, l}(Z) z_{l} \\
& \quad=\widetilde{B}_{j}\left(u_{1}(t), \ldots, u_{n}(t)\right)+\widetilde{g}_{j}(t), \\
& 0 \leq t \leq \tau, j=1, \ldots, n, u_{j}(0)=u_{0, j}, j=1, \ldots, n,
\end{aligned}
$$

where

$$
\begin{aligned}
\widetilde{B}_{j}\left(u_{1}, \ldots, u_{n}\right)= & B_{j}\left(u_{1}, \ldots, u_{n}\right) \\
& -\sum_{l, k=1}^{N} \Phi_{k}\left[B_{j}\left(u_{1}, \ldots, u_{n}\right)\right] \widetilde{\Psi}_{k, l}(Z) z_{l}, \\
& j=1, \ldots, n,
\end{aligned}
$$

$\tilde{g}_{j}(t)$

$=\sum_{k, l=1}^{N}\left\{h_{j, k}^{\prime}(t)-\Phi_{k}\left[g_{j}(t)\right]\right\} \widetilde{\Psi}_{k, l}(Z) z_{l}+g_{j}(t)$,

$$
0 \leq t \leq \tau, j=1, \ldots, n
$$


We list now our assumptions. Let $A_{j}: \mathscr{D}\left(A_{j}\right) \subset X \rightarrow X$, $j=1, \ldots, n$, and assume that each $A_{j}$ satisfy properties (H1) and (H2). $B_{j}$ is a bounded multilinear operator from $\prod_{k=1}^{n} \mathscr{D}\left(A_{k}\right)$ to $X_{A_{j}}^{\theta}, z_{k} \in \bigcap_{j=1}^{n} X_{A_{j}}^{\theta}, u_{0, j} \in \mathscr{D}\left(A_{j}\right)$ and $A_{j} u_{0, j} \in X_{A_{j}}^{\theta},(0<) 3-2 \alpha-\beta<\theta<1, g_{j} \in C\left([0, \tau] ; X_{A_{j}}^{\theta}\right)$, $h_{j, k} \in C([0, \tau] ; \mathbb{R}), j=1, \ldots, n, k=1, \ldots, N$.

As is easily seen there exists a constant $C_{2}$ such that

$$
\begin{gathered}
\left\|\widetilde{B}_{j}\left(u_{1}, \ldots, u_{n}\right)\right\|_{X_{A_{j}}^{\theta}} \leq C_{2} \sum_{k=1}^{n}\left\|A_{k} u_{k}\right\|_{X}, \\
\widetilde{g}_{j} \in C\left([0, \tau] ; X_{A_{j}}^{\theta}\right) .
\end{gathered}
$$

Problem (B.40) is solved by transforming it to the following system of integral equations

$$
\begin{aligned}
u_{j}(t)= & e^{t A_{j}} u_{0, j} \\
& -\int_{0}^{t} \sum_{k, l=1}^{N} \Phi_{k}\left[A_{j} u_{j}(s)\right] \widetilde{\Psi}_{k, l}(Z) e^{(t-s) A_{j}} z_{l} d s \\
& +\int_{0}^{t} e^{(t-s) A_{j}} \widetilde{B}_{j}\left(u_{1}(s), \ldots, u_{n}(s)\right) d s \\
& +\int_{0}^{t} e^{(t-s) A_{j}} \widetilde{g}_{j}(s) d s .
\end{aligned}
$$

Applying $A_{j}$ to both sides one deduces

$$
\begin{aligned}
A_{j} u_{j}(t)= & A_{j} e^{t A_{j}} u_{0, j} \\
& -\int_{0}^{t} \sum_{k, l=1}^{N} \Phi_{k}\left[A_{j} u_{j}(s)\right] \widetilde{\Psi}_{k, l}(Z) A_{j} e^{(t-s) A_{j}} z_{l} d s \\
& +\int_{0}^{t} A_{j} e^{(t-s) A_{j}} \widetilde{B}_{j}\left(u_{1}(s), \ldots, u_{n}(s)\right) d s \\
& +\int_{0}^{t} A_{j} e^{(t-s) A_{j}} \widetilde{g}_{j}(s) d s .
\end{aligned}
$$

Hence one is led to the following system of integral equations for new unknown finctions $v_{j}(t)=A_{j} u_{j}(t), j=$ $1, \ldots, n$ :

$$
\begin{aligned}
v_{j}(t)= & A_{j} e^{t A_{j}} u_{0, j} \\
& -\int_{0}^{t} \sum_{k, l=1}^{N} \Phi_{k}\left[v_{j}(s)\right] \widetilde{\Psi}_{k, l}(Z) A_{j} e^{(t-s) A_{j}} z_{l} d s \\
& +\int_{0}^{t} A_{j} e^{(t-s) A_{j}} \widetilde{B}_{j}\left(A_{1}^{-1} v_{1}(s), \ldots, A_{n}^{-1} v_{n}(s)\right) d s \\
& +\int_{0}^{t} A_{j} e^{(t-s) A_{j}} \widetilde{g}_{j}(s) d s .
\end{aligned}
$$

Let

$$
\begin{gathered}
v_{j, 0}(t)=A_{j} e^{t A_{j}} u_{0, j}+\int_{0}^{t} A_{j} e^{(t-s) A_{j}} \widetilde{g}_{j}(s) d s, \\
v_{j, v+1}(t) \\
=v_{j, 0}(t)-\int_{0}^{t} \sum_{k, l=1}^{N} \Phi_{k}\left[v_{j, v}(s)\right] \widetilde{\Psi}_{k, l}(Z) A_{j} e^{(t-s) A_{j}} z_{l} d s \\
+\int_{0}^{t} A_{j} e^{(t-s) A_{j}} \widetilde{B}_{j}\left(A_{1}^{-1} v_{1, v}(s), \ldots, A_{n}^{-1} v_{n, \nu}(s)\right) d s, \\
v=0,1,2, \ldots
\end{gathered}
$$

In view of (B.43) and [25, Corollary 3.3] one observes

$$
v_{j, 0} \in C^{(\theta-3+2 \alpha+\beta) / \alpha}([0, \tau] ; X) \bigcap B\left([0, \tau] ; X_{A_{j}}^{(\theta-3+2 \alpha+\beta) / \alpha}\right) .
$$

From

$$
\begin{gathered}
v_{j, v+1}(t)-v_{j, v}(t) \\
=-\int_{0}^{t} \sum_{k, l=1}^{N} \Phi_{k}\left[v_{j, v}(s)\right. \\
\left.\quad-v_{j, v-1}(s)\right] \widetilde{\Psi}_{k, l}(Z) A_{j} e^{-(t-s) A_{j}} z_{l} d s \\
+\int_{0}^{t} A_{j} e^{-(t-s) A_{j}} \widetilde{B}_{j}\left(A_{1}^{-1} v_{1, v}(s)\right. \\
-A_{1}^{-1} v_{1, v-1}(s), \ldots, A_{n}^{-1} v_{n, v}(s) \\
\left.-A_{n}^{-1} v_{n, v-1}(s)\right) d s
\end{gathered}
$$

and (B.42) it follows that

$$
\begin{aligned}
& \left\|v_{j, v+1}(t)-v_{j, v}(t)\right\|_{X} \\
& \leq C_{2} \sum_{k, l=1}^{N}\left\|\Phi_{k}\right\|_{X^{*}}\left|\widetilde{\Psi}_{k, l}(Z)\right|\left\|z_{l}\right\|_{X_{A_{j}}^{\theta}} \\
& \quad \times \int_{0}^{t}\left\|v_{j, v}(s)-v_{j, v-1}(s)\right\|_{X}(t-s)^{(\beta-2+\theta) / \alpha} d s \\
& \quad+C_{1} C_{2} \int_{0}^{t}(t-s)^{(\beta-2+\theta) / \alpha} \\
& \quad \times \sum_{k=1}^{n}\left\|v_{k, v}(s)-v_{k, v-1}(s)\right\|_{X} d s .
\end{aligned}
$$

Since $(\beta-2+\theta) / \alpha>-1$, one can easily show that the sequence $\left\{\left(v_{1, v}, \ldots, v_{n, v}\right)\right\}$ converges to a set of functions $\left(v_{1}, \ldots, v_{n}\right)$ uniformly in $X^{n}$ and $\left(v_{1}, \ldots, v_{n}\right)$ is a solution to the system (B.46) of integral equations. It is obvious that a set of functions defined by $\left(u_{1}(t), \ldots, u_{n}(t)\right)=\left(A_{1}^{-1} v_{1}(t)\right.$, $\left.\ldots, A_{n}^{-1} v_{n}(t)\right)$ is a solution to the system (B.44). Again in view 
of $\left[25\right.$, Corollary 3.3] one concludes that $\left(u_{1}(t), \ldots, u_{n}(t)\right)$ is a unique solution to (B.40) satisfying

$$
\begin{aligned}
& u_{j}^{\prime} \in C([0, \tau] ; X) \bigcap B\left([0, \tau] ; X_{A_{j}}^{(\theta-3+2 \alpha+\beta) / \alpha}\right), \\
& A_{j} u_{j} \in C^{(\theta-3+2 \alpha+\beta) / \alpha}([0, \tau] ; X) \bigcap B\left([0, \tau] ; X_{A_{j}}^{(\theta-3+2 \alpha+\beta) / \alpha}\right), \\
& \quad j=1, \ldots, n .
\end{aligned}
$$

\section{Conflict of Interests}

The authors declare that there is no conflict of interests regarding the publication of this paper.

\section{Acknowledgments}

A. Favini and A. Lorenzi are members of G.N.A.M.P.A. of the Italian Istituto Nazionale di Alta Matematica (INdAM).

\section{References}

[1] M. Al Horani and A. Favini, "An identification problem for firstorder degenerate differential equations," Journal of Optimization Theory and Applications, vol. 130, no. 1, pp. 41-60, 2006.

[2] M. Al-Horani and A. Favini, "Degenerate first order identification problems in Banach spaces," in Differential Equations Inverse and Direct Problems, A. Favini and A. Lorenzi, Eds., pp. 1-15, Taylor \& Francis, Boca Raton, Fla, USA, 2006.

[3] A. Favini and A. Yagi, Degenerate Differential Equations in Banach Spaces, Marcel Dekker, New York, NY, USA, 1st edition, 1999.

[4] K. Engel, "Operatormatrices and systems of evolution equations," In press.

[5] A. Yagi, Abstract Parabolic Evolution Equations and Their Applications, Monographs in Mathematics, Springer, Berlin, Germany, 2010.

[6] K. Taira, "The theory of semigroups with weak singularity and its applications to partial differential equations," Tsukuba Journal of Mathematics, vol. 13, no. 2, pp. 513-562, 1989.

[7] V. Capasso and K. Kunisch, "A reaction-diffusion system arising in modelling man-environment diseases," Quarterly of Applied Mathematics, vol. 46, no. 3, pp. 431-450, 1988.

[8] M. Al Horani and A. Favini, "Degenerate first-order inverse problems in Banach spaces," Nonlinear Analysis: Theory, Methods \& Applications, vol. 75, no. 1, pp. 68-77, 2012.

[9] H. Triebel, Interpolation Theory, Function Spaces, Differential Operators, North-Holland, Amsterdam, The Netherlands, 1978.

[10] W. von Wahl, "Lineare und semilineare parabolische Differentialgleichungen in Räumen hölderstetiger Funktionen," Abhandlungen aus dem Mathematischen Seminar der Universität Hamburg, vol. 43, pp. 234-262, 1975.

[11] L. Lorenzi and M. Bertoldi, Analytic Methods for Markov Semigroups, vol. 283 of Pure and Applied Mathematics, Chapman \& Hall/CRC, Boca Raton, Fla, USA, 2007.

[12] I. Lasiecka and R. Triggiani, Control Theory for Partial Differential Equations: Continuous and Approximation Theories I, II: Abstract Parabolic Systems, Cambridge University Press, Cambridge, UK, 2000.
[13] K. Taira, Analytic Semigroups and Semilinear Initial Boundary Value Problems, Cambridge University Press, Cambridge, UK, 1995.

[14] C. Wild, "Semi-groupes de croissance $\alpha<1$ holomorphes," Comptes Rendus de l'Académie des Sciences, vol. 285, no. 6, pp. A437-A440, 1977.

[15] A. Favini, A. Lorenzi, H. Tanabe, and A. Yagi, "An $L^{p}$-approach to singular linear parabolic equations in bounded domains," Osaka Journal of Mathematics, vol. 42, no. 2, pp. 385-406, 2005.

[16] A. Favini, A. Lorenzi, H. Tanabe, and A. Yagi, "An $L^{p}$-approach to singular linear parabolic equations with lower order terms," Discrete and Continuous Dynamical Systems A, vol. 22, no. 4, pp. 989-1008, 2008.

[17] A. Favini, A. Lorenzi, and H. Tanabe, "Degenerate integrodifferential equations of parabolic type with Robin boundary conditions: $L^{2}$-theory," Journal of the Mathematical Society of Japan, vol. 61, no. 1, pp. 133-176, 2009.

[18] S. G. Kreĭn, Linear Differential Equations in Banach Space, American Mathematical Society, Providence, RI, USA, 1971.

[19] E. Sinestrari, "On the abstract Cauchy problem of parabolic type in spaces of continuous functions," Journal of Mathematical Analysis and Applications, vol. 107, no. 1, pp. 16-66, 1985.

[20] A. Lunardi, Analytic Semigroups and Optimal Regularity in Parabolic Problems, Birkhäuser, Basel, Switzerland, 1995.

[21] K. Yosida, Functional Analysis, Springer, Berlin, Germany, 2nd edition, 1969.

[22] H. O. Fattorini, The Cauchy Problem, vol. 18 of Encyclopedia of Mathematics and its Applications, Addison-Wesley, Reading, Mass, USA, 1983.

[23] A. Pazy, Semigroups of Linear Operators and Application to Partial Differential Equations, vol. 44 of Applied Mathematical Sciences, Springer, New York, NY, USA, 1983.

[24] K. Taira, "On the existence of Feller semigroups with discontinuous coefficients. II," Acta Mathematica Sinica (English Series), vol. 25, no. 5, pp. 715-740, 2009.

[25] A. Favini, A. Lorenzi, and H. Tanabe, "Direct and inverse problems for systems of Singular differential boundary-value problems," Electronic Journal of Differential Equations, vol. 2012, no. 255, pp. 1-34, 2012. 


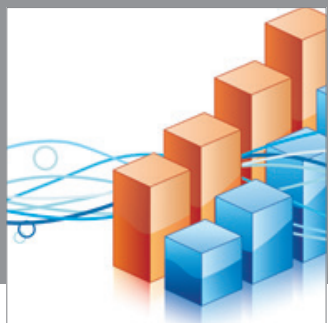

Advances in

Operations Research

mansans

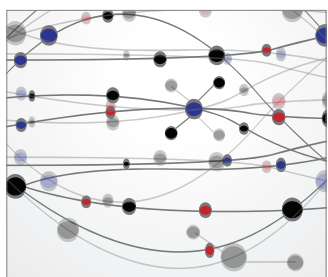

The Scientific World Journal
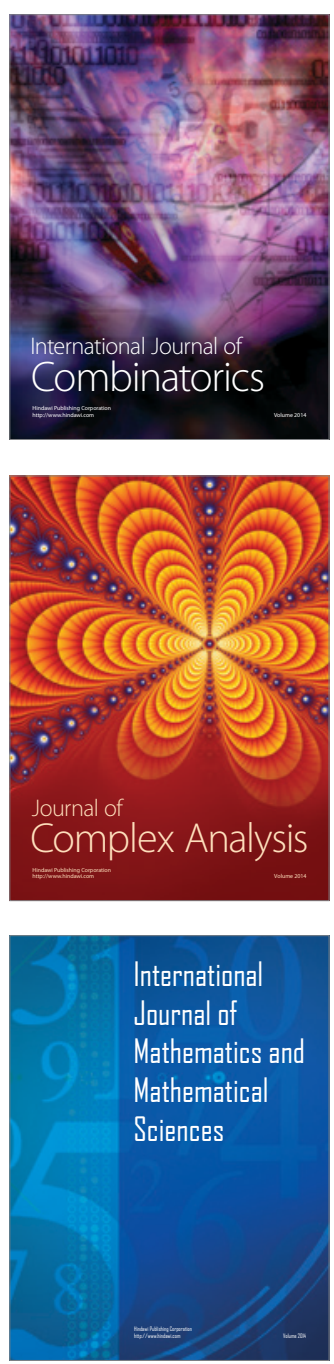
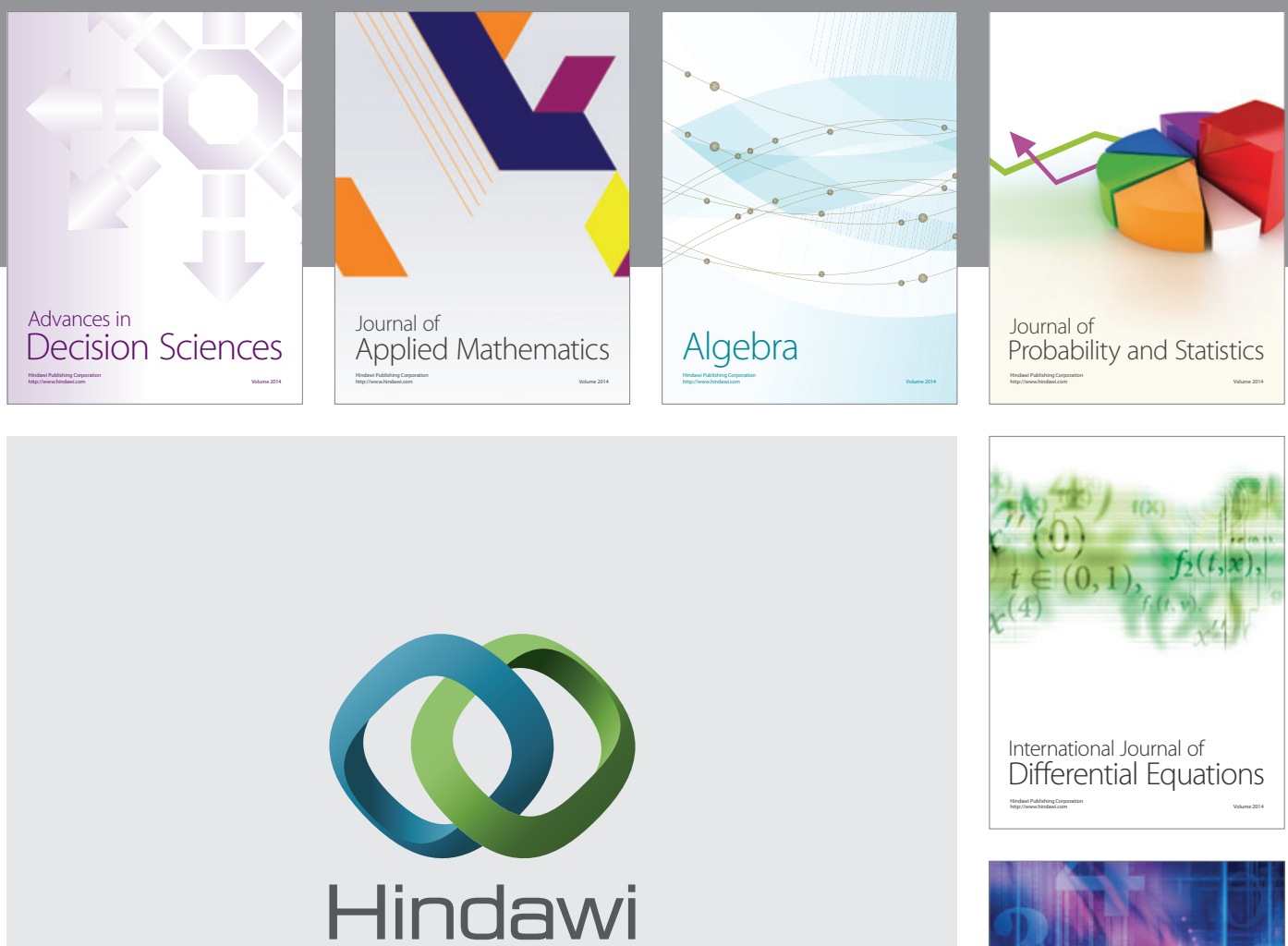

Submit your manuscripts at http://www.hindawi.com
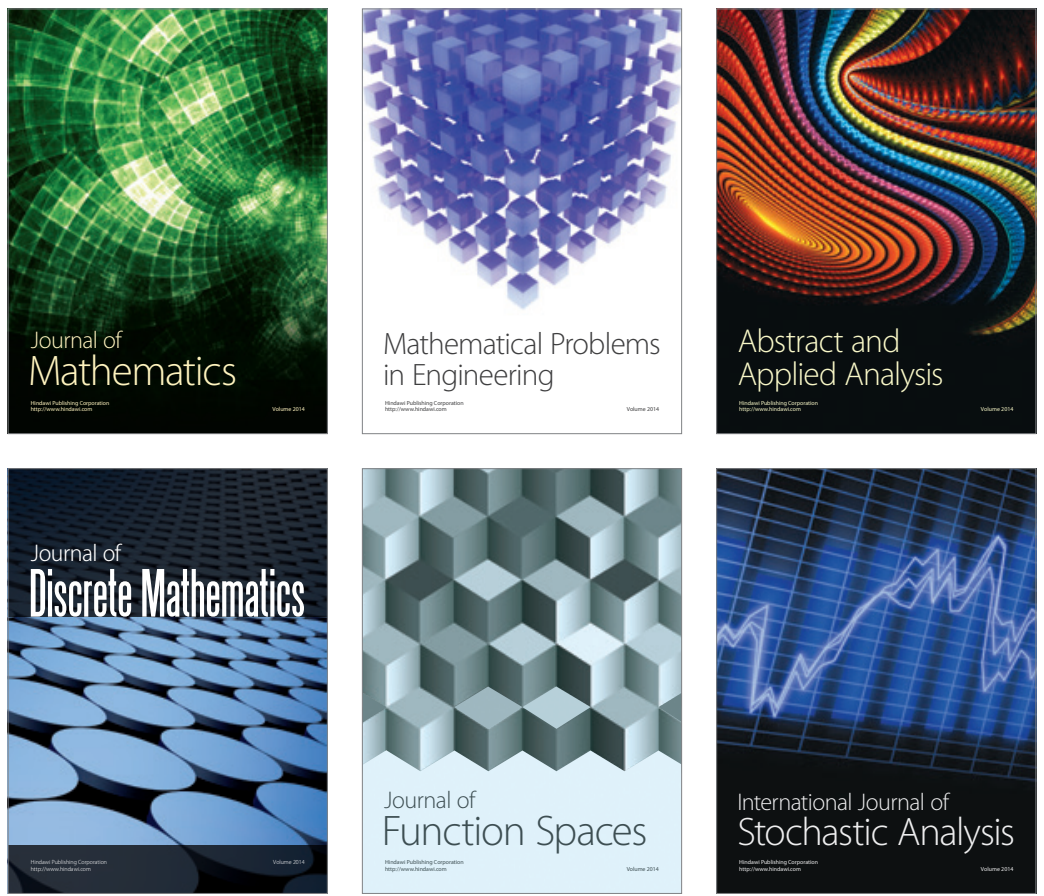

Journal of

Function Spaces

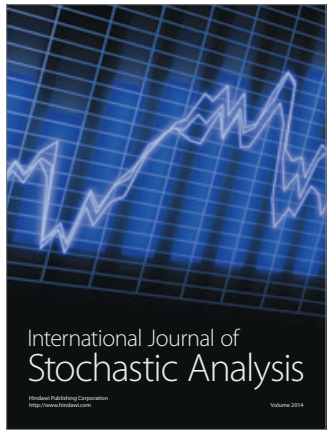

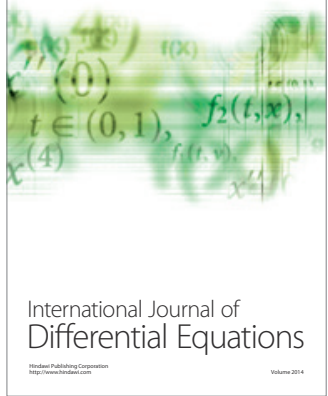
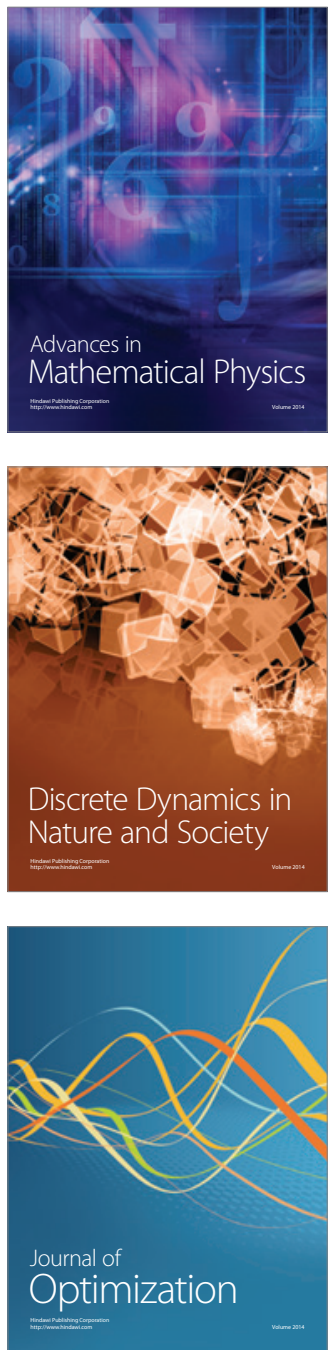